\title{
Constitutive heterogeneity and response diversity of microglia in pathological conditions
}

\author{
Doctoral Thesis \\ In partial fulfillment of the requirements for the degree \\ "Doctor rerum naturalium (Dr. rer. nat.)" \\ in the Molecular Medicine Study Program \\ of the Georg-August University School of Science (GAUSS) \\ at the Georg-August University Göttingen \\ submitted by Ulla Gertig \\ born in Burg
}

Göttingen, 2016 


\section{Members of the Thesis Committee:}

\section{Supervisors}

Prof. Dr. Uwe. K. Hanisch $\dagger$

Department of Neuropathology, University Medical Center Göttingen

Prof. Dr. Wolfgang Brück

Department of Neuropathology, University Medical Center Göttingen

\section{Second member of the thesis committee}

Prof. Dr. Dr. Hannelore Ehrenreich

Department of Clinical Neurosciences, Max Planck Institute of Experimental Medicine, Göttingen

\section{Third member of the thesis committee}

Prof. Dr. Fred Wouters

Department of Neuropathology, University Medical Center Göttingen

\section{Further members of the examination board:}

Prof. Dr. med. Jutta Gärtner

Department of Neuropediatric, University Medical Center Göttingen

Prof. Dr. Ralf Heinrich

Department of Cellular Neurobiology, Schwann-Schleiden-Forschungszentrum, Göttingen

Dr. Hauke Werner

Department of Neurogenetics, Max Planck Institute of Experimental Medicine, Göttingen

Date of Disputation: 12.12.2016 


\section{Affidavit}

Here I declare that my doctoral thesis entitled "Constitutive heterogeneity and response diversity of microglia in pathological conditions" has been written independently with no other sources and aids than quoted.

Ulla Gertig

Göttingen, December 2016 


\section{List of publications}

\section{A hippocampal insulin-growth factor 2 pathway regulates the extinction of fear memories}

Agis-Balboa RC, Arcos-Diaz D, Wittnam J, Govindarajan N, Blom K, Burkhardt S, $\underline{\text { Haladyniak* U, }}$ Agbemenyah HY, Zovoilis A, Salinas-Riester G, Opitz L, Sananbenesi F, Fischer A. 2011. EMBO J 30:4071-4083.

\section{Microglial diversity by responses and responders}

Gertig U, Hanisch U-K. 2014. Front Cell Neurosci 8:101.

CD14 is a key organizer of microglial responses to CNS infection and injury

Janova H, Böttcher C, Holtman IR, Regen T, van Rossum D, Götz A, Ernst A-S, Fritsche C, Gertig U, Saiepour N, Gronke K, Wrzos C, Ribes S, Rolfes S, Weinstein J, Ehrenreich H, Pukrop T, Kopatz J, Stadelmann C, Salinas-Riester G, Weber MS, Prinz M, Brück W, Eggen BJL, Boddeke HWGM, Priller J, Hanisch U-K. 2016. Glia 64:635-649.

*maiden name 


\section{Table of contents}

List of publications $\quad$ IV

Acknowledgments

$\begin{array}{lll}\text { Abstract } & \text { IX }\end{array}$

List of figures $\quad$ X

List of tables $\quad$ XII

List of abbreviations _ XIII

1 Introduction 2

1.1 Microglia ........................ 2

1.2 Origin of microglia . . . . . . . . . . . . . . 2

1.3 Role of microglia in development . . . . . . . . . . . . . . . 3

1.4 Microglia functions . . . . . . . . . . . . . . . . 4

1.5 Microglia signaling . . . . . . . . . . . . . . 5

1.6 Microglia heterogeneity . . . . . . . . . . . . 8

1.6.1 Regional heterogeneity . . . . . . . . . . 8

1.6.2 Functional heterogeneity ............... 9

1.7 Microglia and diseases . . . . . . . . . . . . . . 11

1.8 Aim of the thesis . . . . . . . . . . . . . . . 12 
2 Methods 13

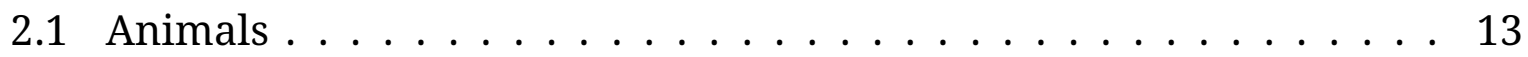

2.2 Primary Mouse Microglia Preparation . . . . . . . . . . . . 13

2.3 L929 fibroblast cultures . . . . . . . . . . . . . . 15

2.4 Cytokine and chemokine measurements . . . . . . . . . 15

2.5 Cell viability assessment . . . . . . . . . . . . . . . 16

2.6 BrdU Cell Proliferation ELISA . . . . . . . . . . . . . . . . . . 16

2.7 Enzyme Linked Immuno Spot Assay (ELISpot) . . . . . . . . . . . 17

2.8 Fluorescence-activated cell sorting (FACS) . . . . . . . . . . . . 18

2.9 Supernatant transfer experiment . . . . . . . . . . . . . . . 19

2.10 Insert experiment . . . . . . . . . . . . . 20

2.11 RNA extraction . . . . . . . . . . . . . . 20

2.12 cDNA synthesis . . . . . . . . . . . . . . . 21

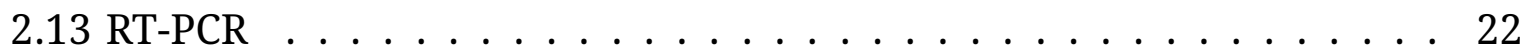

$2.14 \mathrm{LDH}$ assay . . . . . . . . . . . . . . . . 23

2.15 Statistical analysis . . . . . . . . . . . . . . 23

3 Results $\quad 24$

3.1 Subpopulation sizes are dependent on the severity of the challenge 24

3.2 Microglial subpopulation sizes are density dependent . . . . . . . 31

3.3 Microglia subpopulations overlap in their functions . . . . . . . 35

3.4 Microglia proliferation and cytokine secretion . . . . . . . . . . 37

3.5 Effect of environmental cues on microglia subpopulation sizes . . . 41

3.6 TNFa effect on TNFa secretion . . . . . . . . . . . . . . 45 
4 Discussion $\quad 53$

4.1 Microglia subpopulation sizes are dynamically adapted . . . . . . 54

4.2 Microglia subpopulation sizes and behavior are density dependent 57

4.3 Microglia subpopulations are highly complex organized . . . . . . 59

4.4 Microglia are multitasking capable . . . . . . . . . . . . . . . . 61

4.5 Microglia subpopulation size is influenced by environmental cues . 63

4.6 Organization of microglia subpopulation sizes is self-regulated . . . 65

5 Summary and Conclusion $\quad 67$

6 Bibliography $\quad 69$

7 Curriculum vitae $\quad 82$ 


\section{Acknowledgments}

I would like to sincerely thank my supervisor Prof. Dr. Uwe K. Hanisch for giving me the opportunity to work in his laboratory on this wonderful project. It was always inspiring to brainstorm about new ideas, methods and the possible mechanisms behind. I would also like to thank Prof. Dr. Wolfgang Brück for stepping in as a supervisor after the tragic and unexpected death of Prof. Hanisch. Thank you for allowing me to continue my project, for the support, ideas and especially the very time efficient meetings.

Special thanks goes to my thesis committee members Prof. Hannelore Ehrenreich and Prof. Fred Wouters for their supporting comments and ideas, their time and appreciation.

My deepest gratitude goes to my colleagues Insa Dammann, Kim Bleuel, Sarah Traffehn, Nasrin Saiepour, Christin Fritsche and Linda Feldmann. I never would have survived all the hardships, struggles and desperations without you girls. Thank you for the daily coffee, the encouragement and most importantly the free sarcasm. Special thanks goes to Basti for being the substitute during a breakdown once no girl was available ;-)

I am also grateful for the help, support and understanding of our technical assistances Elke Pralle and Susanne Kiecke.

Apart from my colleagues of the neuropathology department, I want to thank my family and friends as well. You were always there for me, cared for me, supported me in every imaginable way and accompanied me during all my studies.

My biggest hug and thanks goes to my beloved husband Michael Gertig. Thank you for enduring me, supporting me, loving me, and for gifting me with our lovely daughter Emily. I would have never made it this far without you. 


\section{Abstract}

Microglia, resident myeloid cells of the CNS, execute an enormous variety of different functions like tissue surveillance, nursing of synapses, phagocytosis of foreign material or debris, and initiation of appropriate immune responses upon CNS infection and damage (Hanisch and Kettenmann, 2007; Kettenmann et al., 2011). The duration of an immune response is thereby selflimiting as it resolves with a successful combat of the threat. A sustaining threat, however, can lead to an over-activation of microglia which switches their initially beneficial effect to a rather detrimental outcome. This disturbed microglia phenotype leads to a variety of neuropsychiatric disorders or neurodegenerative diseases and aggravates the disease outcomes, mostly resulting in neurotoxicity (Gold and Khoury, 2015; Hickman et al., 2008; von Bernhardi et al., 2015). Targeting microglia is therefore a promising therapeutic approach to oppose those diseases (Chen et al., 2014; Wes et al., 2016). Though, microglia functions are in most cases performed by a mere subpopulation, a drug targeting the whole microglia population would thereby effect not only the subsets causing the neurotoxicity but also the subsets which try to resolve the disease (Biber et al., 2016; Marshall et al., 2014; Olah et al., 2012; Venkatesan et al., 2010). Thus, to develop a useful drug, which specifically targets a harmful subpopulation of microglia, the microglial organization principle needs to be unraveled beforehand.

This study focused on the functional heterogeneity of a microglial immune response by investigating cytokine producing microglia subsets confronted with a variety of stimuli conditions in different environmental contexts. I observed a highly specific adaptation of those subsets. The degree of adaptation is thereby dependent on the investigated function, the used stimulus, the stimulus intensity and the microglia density. Further analysis revealed that the subpopulations are not performing one single function but multiple functions at once. Function specific subpopulations are thereby composed of subpopulations which perform other functions as well. These results demonstrate an almost infinite organization complexity of microglia subsets, highlighting the importance of further research to fully understand the nature of microglia. 


\section{List of figures}

1.1 Toll-like receptor signaling $\ldots \ldots \ldots \ldots \ldots \ldots \ldots$

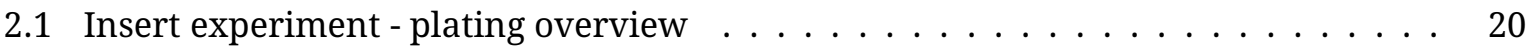

3.1 ELISpot, ELISA and correlation analysis of TNFa- and RANTES-producing microglia

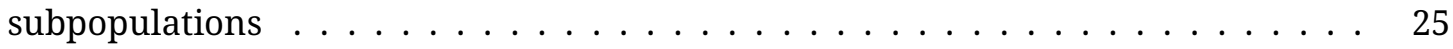

3.2 FACS analysis of TNFa- and RANTES-producing microglia subpopulations . . . . . . 27



3.4 Spot intensity and spot size $\ldots \ldots \ldots \ldots \ldots \ldots$

3.5 ELISpot analysis of TNFa-producing microglia populations after combined stimu-

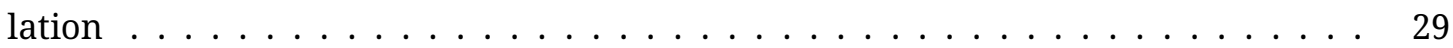

3.6 Density dependency of microglia subpopulations $\ldots \ldots \ldots \ldots \ldots$

3.7 Influence of the cell density on the microglia subpopulation sizes and cell death . . 33

3.8 Density dependent secretion per cell and subpopulation increase $\ldots \ldots \ldots \ldots$. . . 34

3.9 Composition of TNFa- and RANTES-producing microglia subpopulation sizes . . . . 35

3.10 Proliferation rate of challenged microglia isolated from WT and transgenic mice . 37

3.11 Determination of TNFa-producing and proliferating microglia subpopulation sizes $\quad 39$

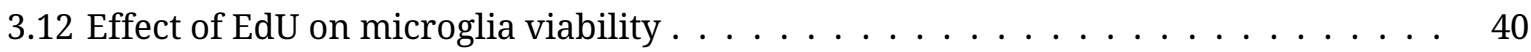

3.13 Determination of supernatant effect on TNFa- producing microglia subpopulation

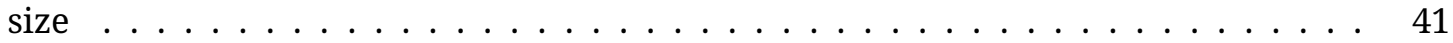

3.14 Cytokine levels inside and outside of inserts $\ldots \ldots \ldots \ldots \ldots$. . . . . . . 42

3.15 Supernatant transfer experiments $\ldots \ldots \ldots \ldots$. . . . . . . . . . . . . . . 43 
3.16 Time dependent effect of TNFa pre-incubations $\ldots \ldots \ldots \ldots$

3.17 Time dependent effect of RANTES pre-incubations $\ldots \ldots \ldots$. . . . . . . . . 47

3.18 Time dependent effect of MCP-1 pre-incubations $\ldots \ldots \ldots \ldots \ldots$. . . . . 48

3.19 Time dependent effect of MIP1a pre-incubations $\ldots \ldots \ldots$. . . . . . . . . . . 49

3.20 Effect of TNFa on microglia viability and RNA levels of cytokines . . . . . . . . . 50

3.21 TNFa pre-incubation effect on TNFa- and RANTES-producing microglia subpopula-



3.22 Effect of TNFa pre-incubation after 8 hours of LPS stimulation . . . . . . . . . . . 52 


\section{List of tables}

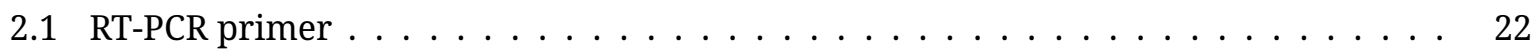

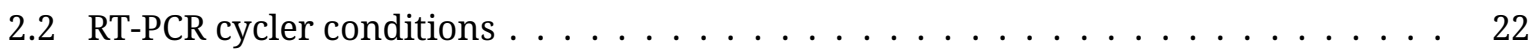

$3.1 \mathrm{R}^{2}$ of cytokine producing subpopulation sizes and cytokine secretion $\ldots \ldots \ldots$

$3.2 \mathrm{R}^{2}$ of TNFa producing subpopulation sizes vs. microglia density $\ldots \ldots \ldots$. . . . . 32 


\section{List of abbreviations}

\begin{tabular}{ll} 
BrdU & Bromodeoxyuridine \\
Cd11b-APC & CD11b-Allophycocyanin \\
CD11b-PB & CD11b-Pacific Blue \\
cDNA & Complementary deoxyribonucleic acid \\
CNS & central nervous system \\
CpG & cytidine-phosphate guanosine \\
DAMPs & damage/danger associated molecular patterns \\
DMEM & Dulbecco's modified Eagle's medium \\
DNA & Deoxyribonucleic acid \\
DNase & deoxyribonuclease \\
dsRNA & double stranded RNA \\
EdU & 5-ethynyl-2'-deoxyuridine \\
ELISA & enzyme-linked immunosorbent assay \\
ELISpot & Enzyme Linked Immuno Spot Assay \\
FACS & Fluorescence-activated cell sorting \\
FCS & fetal calf serum \\
FN & Fibronectin \\
HBSS & Hanks balanced salt solution \\
IL-1 $\beta$ & interleukin 1 $\beta$ \\
IL-4 & interleukin 4 \\
IL12-p40 & Interleukin 12 \\
IL6 & interleukin 6 \\
IRF7 & interferon regulatory factor 7 \\
KC & keratinocyte-derived chemokine \\
LDH & Lactate dehydrogenase \\
LPS & Macterial lipopolysaccharide \\
MALP-2 & monocyte chemotactic protein 1 \\
\hline
\end{tabular}




$\begin{array}{ll}\text { MD2 } & \text { myeloid differentiation factor 2 } \\ \text { MHC } & \text { Major histocompatibility complex } \\ \text { MIP1a } & \text { macrophage inflammatory protein 1a } \\ \text { MyD88 } & \text { myeloid differentiation primary response gene 88 } \\ \text { NF-kB } & \text { nuclear factor kB } \\ \text { PAMPs } & \text { pathogen-associated molecular patterns } \\ \text { PBS } & \text { phosphate buffered saline } \\ \text { PLL } & \text { Poly-L-Lysin } \\ \text { PRRs } & \text { pattern-recognition receptors } \\ \text { RANTES } & \text { regulated upon activation normal T-cell expressed and presumably secreted } \\ \text { RANTES-PE } & \text { RANTES-Phycoerythrin } \\ \text { RD1 } & \text { reagent diluent 1 } \\ \text { RNA } & \text { Ribonucleic acid } \\ \text { RT } & \text { room temperature } \\ \text { RT-PCR } & \text { Real-Time Polymerase chain reaction } \\ \text { ssRNA } & \text { single stranded RNA } \\ \text { TIR } & \text { Toll-interleukin 1 (IL-1) receptor } \\ \text { TIRAP } & \text { TIR domain-containing adaptor protein } \\ \text { TLRs } & \text { Toll-like receptors } \\ \text { TNFa } & \text { Tumor necrosis factor a } \\ \text { TRAM } & \text { TRIF-related adaptor molecule } \\ \text { TREM2 } & \text { triggering receptor expressed on myeloid cells 2 } \\ \text { TRIF } & \text { TIR-domain containing adaptor protein inducing Interferon- } \beta \\ \text { UMG } & \text { University Medical Center Göttingen } \\ \text { WST-1 } & \text { water soluble tetrazolium salts } \\ & \end{array}$




\section{Introduction}

\subsection{Microglia}

Microglia are brain-resident myeloid cells and execute an enormous variety of different functions within the central nervous system (CNS) including surveillance of the tissue, nursing of synapses, phagocytosis of debris, cells or foreign material, and initiation of appropriate immune responses upon damage or infection. They are highly motile cells involved in proper brain development but also implicated with a variety of neurodegenerative and inflammatory diseases (Hanisch and Kettenmann, 2007; Kettenmann et al., 2011). Microglia vary by local densities and functional properties which makes them a very heterogeneous population of cells with the potential to perform their huge repertoire of functions simultaneously by multitasking and division of labor (Gertig and Hanisch, 2014; Hanisch and Kettenmann, 2007; Lawson et al., 1990).

\subsection{Origin of microglia}

Microglia are the prime innate immune cells of the CNS, comprising approximately $10 \%$ of its total cell number (Aguzzi et al., 2013). They form a robust, long living and self-renewing population of immune cells (Kierdorf et al., 2013) with no contribution from bone-marrow derived progenitors under physiological conditions (Ajami et al., 2007). In a healthy environment microglia turnover is rather rare, but if microglia are depleted from brain or spinal cord they are able to repopulate completely within 14 days from a small population of surviving microglia or nestin-expressing microglial progenitor cells (Bruttger et al., 2015; Elmore et al., 2014; Yao et al., 2016).

Unlike neurons and other glial cells which derive from the neuroectoderm, microglia are of mesodermal origin (Chan et al., 2007; Prinz and Mildner, 2011). They derive from erythromyeloid precursor cells in the yolk sac which transform into yolk sac macrophages during primitive hematopoiesis and subsequently migrate into the brain where they become microglia cells (Ginhoux et al., 2010; Hoeffel et al., 2015; Kierdorf et al., 2013; Masuda and Prinz, 2016). As 
microglia start to colonize the murine brain already at embryonic day 8.5-9.5 they play a crucial role in shaping the CNS during development (Ginhoux et al., 2010).

\subsection{Role of microglia in development}

During embryogenesis, microglia are already present in the brain when neurons are still in the process of migration, proliferation and differentiation. During this time, approximately $50 \%$ of the newborn neurons undergo programmed cell death and do not become mature (Schafer and Stevens, 2015). This process involves microglia which are responsible for phagocytosing these newly generated non-surviving cells, as it was shown by various studies conducted in mice, rats and zebrafish (Ferrer et al., 1990; Peri and Nüsslein-Volhard, 2008; Sierra et al., 2010).

Additionally, microglia regulate the number of neural precursor cells in the developing cerebral cortex by phagocytosing a proportion of viable cells with no signs of cell death, apoptosis or nuclear breakdown (Cunningham et al., 2013). In contrast, some findings also indicate a neuroprotective role of microglia necessary for the survival of specific types of neurons (Ueno et al., 2013). An in vitro study was even able to demonstrate the involvement of microglia on neural precursor cell differentiation (Aarum et al., 2003), which was later confirmed in vivo (Xavier et al., 2015).

Microglia are crucial for the synaptic maturation as well. Microglial processes reach developing synapses and remove redundant ones in a process called "synaptic pruning”. A deficiency in this function leads to a rather immature brain circuitry and is assumed to be involved in neurodevelopmental and neuropsychiatric disorders (Hoshiko et al., 2012; Paolicelli et al., 2011; Schafer et al., 2012; Squarzoni et al., 2014; Zhan et al., 2014). A similar function of microglia called "synaptic stripping" which persists beyond development removes synapses from injured neurons (Kettenmann et al., 2013). Studies on mice suggest an additional role of microglia in the modification and elimination of synaptic structures upon alterations in visual experience and multiple learning tasks, indicating a contribution to learning and memory (Parkhurst et al., 2013; Tremblay et al., 2010).

Other studies have shown that microglia support neurogenesis and oligodendrogenesis by 
secreting specific cytokine mixtures into the subventricular zone of developing rat brains (Shigemoto-Mogami et al., 2014). However, apart from their role in the developing brain, microglia exhibit also important functions to maintain the CNS homeostasis through lifetime. The support of neurogenesis, for instance, lasts even beyond embryogenesis since microglia are required for proper adult neurogenesis as well (Ziv et al., 2006).

\subsection{Microglia functions}

Under normal physiological conditions microglia have a ramified appearance that is characterized by extended, branched processes. Even though the somata are rather fixed, their processes are highly motile. They extend and withdraw continually, allowing the microglia to scan the whole brain parenchyma for signs of disturbance within hours (Davalos et al., 2005; Nimmerjahn et al., 2005). Simultaneously, they monitor synapse function of neurons and are involved in their elimination and restoration (Wake et al., 2009).

It is assumed that once microglia recognize an injured cell, debris or even a dying cell, they respond appropriately by repair, support or phagocytosis (Hanisch and Kettenmann, 2007; Nimmerjahn et al., 2005; Streit, 2002). However, when microglia recognize tissue injury or infection, they shift rapidly from their surveilling state to a rather activated phenotype (Davalos et al., 2005). In this state, microglia have a more amoeboid appearance which allows them to migrate actively to the side of injury or infection by following chemotactic gradients (Davalos et al., 2005; Haynes et al., 2006; Honda et al., 2001; Koizumi et al., 2007; Liu et al., 2009). Once microglia arrive at the site of disruption, they start to initiate a range of situation-specific activities like phagocytosis, proliferation, antigen presentation or secretion of cytokines and chemokines in order to recruit additional immune cells from the periphery (Buttini et al., 1996; Graeber et al., 1988; Hanisch, 2002; Kettenmann et al., 2011; Streit, 2002; Streit et al., 1989). To be able to detect all kinds of threats and to adapt a proper immune response, microglia express a vast range of different receptors allowing this discrimination. 


\subsection{Microglia signaling}

In order to sense any threat in form of pathogen-associated molecular patterns (PAMPs) or damage/danger associated molecular patterns (DAMPs), microglia express pattern-recognition receptors (PRRs) (Saijo and Glass, 2011). These receptors are able to recognize lipids, lipoproteins, proteins and nucleic acids derived from bacteria, viruses, parasites and fungi as well as intra- or extracellular proteins released by injured cells. One form of these PRRs are Toll-like receptors (TLRs), which are also present in microglia.

TLRs are transmembrane proteins that have leucine-rich repeats on their extracellular domains to recognize PAMPs and Toll-interleukin 1 (IL-1) receptor (TIR) domains on their intracellular domains to transduct downstream signaling (Kawai and Akira, 2010). There are 12 functional TLRs in mouse (TLR1-TLR9; TLR11-TLR13) and 10 in humans (TLR1-TLR10) (Akira et al., 2006).

TLRs divide into two subgroups depending on their cellular localization and the patterns they recognize. The first group resides on the cell surface and consists of TLR1, TLR2, TLR4, TLR5, TLR6 and TLR11. Those TLRs recognize mainly microbial membrane components. The second group comprising TLR3, TLR7, TLR8 and TLR9 are exclusively intracellular, expressed on endosomes, the endoplasmic reticulum, endolysosomes or lysosomes, where they recognize mainly microbial nucleic acids. TLRs can signal through two routes, they use either the MyD88 (myeloid differentiation primary response gene 88) dependent signalling pathway which is used by all TLRs except for TLR3 or the TRIF (TIR-domain containing adaptor protein inducing Interferon$\beta$ ) dependent signaling pathway which is used by TLR4 and TLR3. TLR4 is thereby an exception, since it is able to signal through both pathways (Takeda and Akira, 2004).

TLR4 recognizes bacterial lipopolysaccharide (LPS), a cell wall component of gram-negative bacteria such as escherichia coli (Hoshino et al., 1999; Poltorak et al., 1998). To do so, the receptor forms a complex with MD2 (myeloid differentiation factor 2), which allows binding of LPS (Schromm et al., 2001; Shimazu et al., 1999). A multidimer composed of two copies of the afore mentioned LPS-receptor complex recruits the TIR domain-containing adaptor protein (TIRAP) and MyD88 which initiates the signaling for early-phase activation of NF-kB (nuclear factor $\mathrm{kB}$ (Akira et al., 2006). A subsequent internalization of the receptor complex recruits TRAM 
(TRIF-related adaptor molecule) and TRIF and initiates late-phase activation of NF- $\mathrm{KB}$ and induction of type I interferons. Activation of NF- $\mathrm{kB}$ through the described MyD88- and TRIF-dependent pathways regulates inflammatory processes by the induction of inflammatory cytokines (see figure 1.1, p.7) (Kawai and Akira, 2010) .

TLR2 forms heterodimers with either TLR1 or TLR6 (Ozinsky et al., 2000). Upon binding of triacyl-lipopeptides from Gram-negative bacteria and mycoplasma (TLR2-TLR1 heterodimer) or of diacyl-lipopeptides from Gram-positive bacteria and mycoplasma (TLR2-TLR6 heterodimer), both heterodimers signal through the MyD88-dependent pathway to induce the production of inflammatory cytokines (Jin et al., 2007; Kawai and Akira, 2010; Schwandner et al., 1999; Takeuchi et al., 1999).

TLR3 recognizes mainly double stranded RNA (dsRNA) from viruses and virus infected cells (Alexopoulou et al., 2001). It signals through the TRIF-dependent pathway to express type I interferons and inflammatory cytokines (Akira et al., 2006).

TLR5 is known to recognize flagellin, a protein produced by flagellated bacteria. It forms a homodimer and induces NF-kB-dependent production of cytokines by the recruitment of MyD88 (Hayashi et al., 2001).

TLR7 and TLR8 detect single stranded RNA (ssRNA) from viruses and recruit MyD88 for the induction of cytokine secretion and interferon regulatory factor 7 (IRF7) for the induction of type I interferons (Heil et al., 2004; Hemmi et al., 2002).

TLR9 signals the same way as TLR7 and TLR8 but detects unmethylated cytidine-phosphate guanosine (CpG) DNA from bacteria and viruses (Hemmi et al., 2000).

This repertoire of different receptors is necessary to induce situation specific immune responses by microglia. However, a whole range of other receptor proteins besides TLRs are expressed in microglia, but not all receptors are expressed in each cell which leads ultimately to the formation of subpopulations carrying different receptor combinations. This heterogeneity of microglia is not restricted to the expression of receptors, in fact, microglia heterogeneity can be found in nearly each area of investigation. 


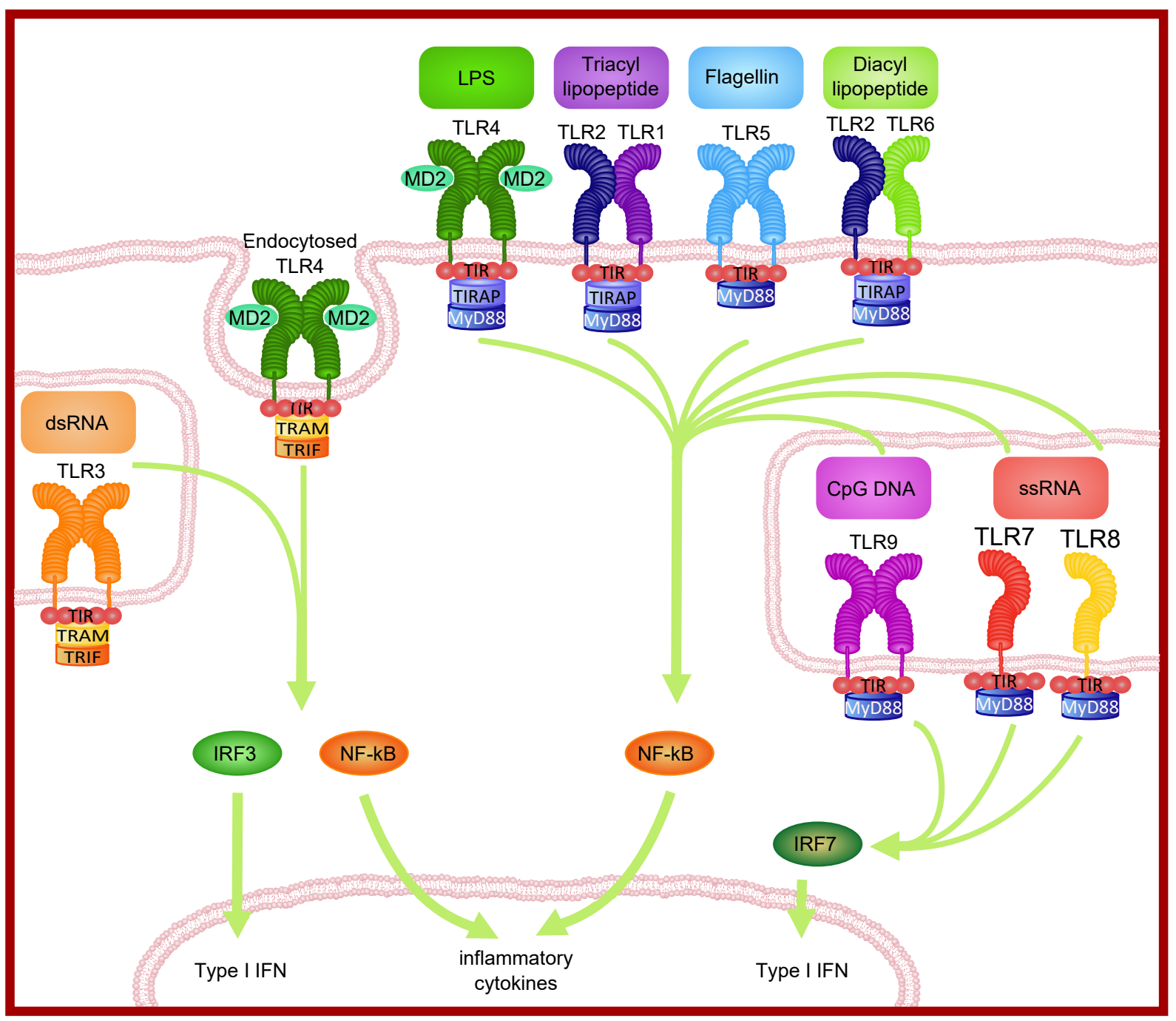

Figure 1.1 - Toll-like receptor signaling: TLR4 binds LPS by forming a complex with MD2. A multidimer composed of two copies of this LPS-receptor complex recruits the TIR domain-containing adaptors TIRAP and MyD88 which initiates the signaling for activation of NF- $\mathrm{kB}$. A subsequent internalization of the receptor complex recruits TRAM and TRIF and initiates once more activation of NF- $\mathrm{B}$ which leads to the induction of inflammatory cytokines. Additional activation of IRF3 induces inductions of type I interferon. TLR2 forms heterodimers with either TLR1 or TLR6. Both heterodimers signal through the MyD88-dependent pathway to induce the production of inflammatory cytokines upon binding of triacyl lipopeptides or diacyl lipopeptides. TLR3 recognizes mainly dsRNA. It signals through the TRIF dependent pathway to recruit type I interferons and inflammatory cytokines. TLR5 forms a homodimer after recognition of flagellin. It induces NF- $\mathrm{B}$ dependent production of cytokines by the recruitment of MyD88. TLR7 and TLR8 detect ssRNA whilst TRL9 detects CpG DNA. They recruit MyD88 and IRF7 to induce cytokine secretion and production of type I interferons, respectively. 


\subsection{Microglia heterogeneity}

Microglia contribute simultaneously to proliferation, maturation, survival and cell death within the CNS. To perform these various activities, it is likely that microglia fall into subpopulations depending on brain region and/or function.

\subsubsection{Regional heterogeneity}

Even though microglia are present in the whole CNS they are not uniformly distributed (Lawson et al., 1990). As a result, microglia densities vary highly within the individual brain regions. It was already shown in 1990 that mouse microglia compose approximately $5 \%$ of all cells in the cerebral cortex and corpus callosum. In the whole brain, this proportion rises regiondependently up to $12 \%$ as seen in the substantia nigra (Lawson et al., 1990). This regiondependent microglia density was also demonstrated in the healthy human brain parenchyma where the proportion of microglia ranged from $0.5 \%$ up to $16 \%$ (Mittelbronn et al., 2001). Recent data show that there is not only regional heterogeneity in microglia density but also in their gene expression profile (Grabert et al., 2016). Genome-wide analysis of microglia from cerebellum, hippocampus, cortex, and striatum of mice indicate that microglia from the first two regions have a more immune-alerted state than the latter two, and that they show a greater expression of genes involved in energy metabolism (Grabert et al., 2016).

It was also shown, that neurons of different brain regions show diverse susceptibility to LPSinduced neurotoxicity which is most probably related to the different abundance of microglia in those regions (Kim et al., 2000). Other studies which investigated the expression of various microglial immunoregulatory markers indicated that the expression level of each marker was highly heterogeneous even though all of them were expressed within the tested brain regions (de Haas et al., 2008; Ren et al., 1999; Smith et al., 2013). Investigation of several microglial purinergic P2X receptors revealed not only a brain-region specific heterogeneity of their expression levels, but also an age- and even a sex-specific dependency (Crain and Watters, 2015). The high complexity of regional microglia heterogeneity was even more validated by a study conducted in the olfactory bulb of rats which could demonstrate that microglia expression levels of 
various markers differed even between the different layers of the tissue, highlighting the impact of the microenvironment (Schmid et al., 2009; Wu et al., 1997).

\subsubsection{Functional heterogeneity}

Even though it was already shown in the 1990s that only subpopulations of microglia express immunologically important molecules like Major histocompatibility complex (MHC) class II (Streit and Graeber, 1993) or specific neurotrophins (Elkabes et al., 1996), it was long believed that all microglia behave or react rather homogeneously. The functional heterogeneity became more and more evident within the recent years and draws increasing attention.

As such, it is nowadays known that the most essential microglial functions are performed by mere subsets of microglia rather than the entire population at once. The phagocytosis of myelin is only one example of these functions, performed by a subpopulation of microglia and plastic in regards to the size of their population (Venkatesan et al., 2010). Microglia can also be classified into proliferative and non-proliferative populations with differing sizes, dependent on the respective brain region and on the surrounding cellular environment (Marshall et al., 2014).

The expression patterns of receptors were found to be organized in a subpopulational manner as well since only a fraction of microglia in rodents express $\mathrm{GABA}_{\mathrm{B}}$, glutamate-, dopamineor noradrenaline-receptors (Färber et al., 2005; Kuhn et al., 2004; Noda et al., 2000). Interestingly, the fraction of cells that express these receptors varies with microglial activity. In accordance, the highly important immunological factor TREM2 (triggering receptor expressed on myeloid cells 2) is only expressed in subpopulations of resting microglia and its expression is brain-region- and most likely microenvironment-dependent (Schmid et al., 2002).

Microglia sensitivity towards neurotransmitters and neurohormones like histamine, serotonine, somatostatin, angiotension II or dopamine is conveyed via subpopulations as well. The neurotransmitter/neurohormone-dependent subpopulations can represent up to $20 \%$ of all microglia, and while some of the subpopulations are just able to react to one substance, some can even react to two or three substances after sequential exposure (Pannell et al., 2014b).

An advancing knowledge on microglia subpopulations may also increase our understanding of 
a variety of central nervous diseases. In a mouse model of Alzheimer's disease, for instance, application of IL-4 (interleukin 4) leads to the expression of CD11c in a subpopulation of microglia that specifically counteracts the inhibitory effects of beta-amyloid on microglia leading to improved plaque phagocytosis and increased neurogenesis (Butovsky et al., 2006). In an ischemic stroke mouse model, researchers identified distinct subpopulations of microglia producing TNFa (Tumor necrosis factor $\alpha$ ) or IL-1 $\beta$ (interleukin $1 \beta$ ) respectively after stroke induction (Clausen et al., 2008). Keratan sulfate, an inhibitor of axonal regeneration, was also demonstrated to be expressed by a microglia subpopulation in a mouse model of Wallerian degeneration (Shinjo et al., 2014). In Hoxb8 mutant mice, the transgene is expressed by a subpopulation of about $40 \%$ of microglia and a loss of function mutation in this gene leads to excessive grooming and sensory defects. This phenotype is similar to the obsessive-compulsive disorder which was observed in humans (Chen et al., 2010). Taken together, studies regarding microglia subsets strongly suggest a vast impact of microglia heterogeneity on the CNS immune system but also subsequent functions of the CNS (Baalman et al., 2015; Bisht et al., 2016; Fang et al., 2014; Kobayashi et al., 2013; Kocur et al., 2015; Pannell et al., 2014a; Pepe et al., 2014; Trias et al., 2013; Wlodarczyk et al., 2015).

Apparently, the findings on functional heterogeneity in microglia are not limited to rodents, as the complexity of microglial organization was successfully demonstrated in a study using microglia cultures from different human donors as well, since the culture cells showed similar gene profiles but very diverse responses to a challenge. The differences elicited by the same challenge even resulted in opposed gene expression changes between the cultures (Meeuwsen et al., 2005).

The heterogeneity of microglia is not only restricted to areas, functionalities or expression patterns, but also dependents on age. Studies conducted in young vs. old mice showed an altered pro-inflammatory profile that comes with age (Godbout et al., 2005; Ritzel et al., 2015; Sierra et al., 2007). These age-dependent alterations, which include microglial functions, self-renewal and vitality are collectively termed as "microglial senescense" (Streit, 2006). Interestingly, this phenomenon as well as other microglia features are linked to a variety of neurodegenerative diseases in which microglia seem to play an essential role (Block and Hong, 2005; Hickman et al., 2008; Prokop et al., 2013). 


\subsection{Microglia and diseases}

Microglia are constantly screening the brain parenchyma for threats and disturbances (Nimmerjahn et al., 2005). Once they encounter a disruption of homeostasis, i.e. due to a pathology, they try to resolve it (Davalos et al., 2005). Though, microglia stay activated as long as the pathology sustains this could switch beneficial to detrimental outcomes or vice versa. In Alzheimer's disease for instance, microglia are able to clear the toxic amyloid beta that accumulates in affected brains. However, if they are confronted with amyloid beta continuously, they lose the ability to clear it. This results in microglial activation, a massive release of pro-inflammatory cytokines and production of reactive oxygen species, that ultimately lead to neurotoxicity and neurodegeneration (Gold and Khoury, 2015; Hickman et al., 2008). This over-activation of microglia was also demonstrated in other neurodegenerative diseases like multiple sclerosis, frontotemporal lobe dementia, Parkinson's disease, hypoxia, stroke and amyotrophic lateral sclerosis (reviewed in Block and Hong, 2005).

Even though the total impact of microglial activation on those diseases is still under investigation, it is already clear that targeting microglia to oppose the diseases is very promising (Chen et al., 2014; Wes et al., 2016). Nonetheless, using anti-inflammatory drugs has a lot of side-effects since they are targeting the whole system, outmaneuvering even the beneficial functions (Biber et al., 2016; Olah et al., 2012). Thus, understanding microglia as a heterogeneous population of immune cells and identification of respective markers to differentiate between the subgroups would be crucial to counteract the strong inflammatory response in those diseases. The development of drugs or tools that are specifically targeting subpopulations of interest while leaving the others unaffected would be of most benefit (Ferreira and Bernardino, 2015). 


\subsection{Aim of the thesis}

Even though the consciousness of microglial heterogeneity is advancing, research specifically investigating microglial subpopulations is still in it's infancy. So far, most studies that identified microglial subgroups were primarily interested to address completely different issues. Thus, the existence of microglial subpopulations is known and mostly accepted, their organization and behavior, however, is completely unknown. Thus, this study aimed to investigate the organization of specific subpopulations and their behavior in different conditions. By focusing on two main functions of microglia, namely cytokine secretion and proliferation, I addressed the following research questions:

1. How do microglia subpopulations behave in front of a challenge? Is an increased cytokine release caused by an increased cell number or by a higher cytokine production per cell?

2. Can microglia subpopulations secrete multiple cytokines? How are the cytokine-specific subgroups organized?

3. Occurs secretion and proliferation simultaneously in the same microglia cell?

4. How are microglia subpopulation sizes organized? 


\section{Methods}

\subsection{Animals}

C57Bl/6J wildtype mice were bred and housed under normal conditions in the Central Animal Facility of the University Medical Center Göttingen (UMG). Mutant strains (myd88-/- and trif ${ }^{l p s}$, both on $\mathrm{C} 57 \mathrm{Bl} / 6 \mathrm{~J}$ background) were bred and maintained under specific pathogen-free condi-

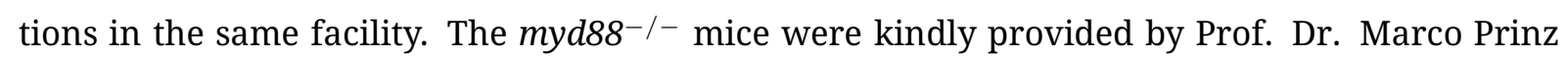
(Department of Neuropathology, University of Freiburg, Germany) and described by Adachi and colleagues (Adachi et al., 1998). The trif ${ }^{p s 2}$ mice were provided by Dr. Bruce Beutler (University of Texas Southwestern Medical Center, USA), carrying a mutation in the C terminus of TRIF which abolishes TRIF-mediated functions (Hoebe et al., 2003). All animals were supplied with food and water ad libitum. Experiments were performed in accordance with the guidelines for animal welfare and protection of the University Göttingen and the German Animal Protection Law.

\subsection{Primary Mouse Microglia Preparation}

Primary cultures were prepared from whole brains of newborn (P0/P1) wildtype C57Bl/6J, or myd88-/- and trif $f^{p s 2}$ mutant mice as described by Regen et al. 2011. Mice were decapitated, the brains were extracted and immediately transferred into petri-dishes containing ice-cold Hanks balanced salt solution (HBSS) (Biochrom; \#L2012). Following the removal of blood vessels and meninges from the brains with help of a binocular stereo microscope, brain tissue was collected in a Falcon tube containing ice-cold HBSS. After being washed trice, the brains were digested with $100 \mu \mathrm{l}$ 2.5\% Trypsin/brain (Biochrom; \#L2133). The tubes were gently vortexed and incubated for 10 minutes in a $37^{\circ} \mathrm{C}$ water bath with additional vortexing in between. The digestion was stopped by addition of $1 \mathrm{ml} / \mathrm{brain}$ medium (Dulbecco's modified Eagle's medium (DMEM) (Gibco; \#41965-062)) containing 10\% fetal calf serum (FCS) (Invitrogen), 100 U/ml penicillin, and 
$100 \mu \mathrm{g} / \mathrm{ml}$ streptomycin (Biochrom; \#A2213). Next, $40 \mu \mathrm{l} / \mathrm{brain}$ deoxyribonuclease (DNase) (Worthington; \#2139) was added to avoid excessive DNA aggregation. Brain structures were dissolved carefully by pipetting up and down several times. A subsequent incubation for 3 minutes at $37^{\circ} \mathrm{C}$ in a water bath ensured a complete decomposition of the tissue. Afterwards, samples were centrifuged at $900 \mathrm{rpm}\left(163 \mathrm{~g}\right.$ ) for 10 minutes at $4^{\circ} \mathrm{C}$. For culturing, the cells were resuspended in 15 $\mathrm{ml}$ medium/2 brains in a F75 cell culture flask (Greiner; \#658175). Flasks were pre-coated with Poly-L-Lysin (PLL) (Sigma Aldrich; \#P1274). To pre-coat the flasks with PLL, they were incubated for 20 minutes with $10 \mathrm{ml}$ PLL and washed thrice with sterile ddH2O and once with medium. Flasks containing cells were incubated overnight at $37^{\circ} \mathrm{C}$ with $5 \% \mathrm{CO}_{2}$.

The next day, cells were washed trice with phosphate buffered saline (PBS) (Gibco; \#14190-094) and once with medium. Cells were washed again with medium on day 3 and 4. On day 9, cells were shaken for 20 minutes at $37^{\circ} \mathrm{C}$ at $901 / \mathrm{min}$ and the medium was substituted with $10 \mathrm{ml}$ fresh medium and $5 \mathrm{ml}$ conditioned L-929 medium. The first microglia could be harvested on day 14 . Therefore, flasks were shaken for at least $30 \mathrm{~min}$ at $37^{\circ} \mathrm{C}$ at $900 \mathrm{rpm}(163 \mathrm{~g})$ and the microglia containing supernatant was transferred into a falcon tube. The remaining cells in the flask were supplied with $10 \mathrm{ml}$ medium and $5 \mathrm{ml} \mathrm{L}-929$ medium and incubated at $37^{\circ} \mathrm{C}$ with $5 \% \mathrm{CO}_{2}$ till the next harvest. The tube containing the microglia was centrifuged at $4{ }^{\circ} \mathrm{C}$ and $900 \mathrm{rpm}$ (163 g) for $10 \mathrm{~min}$. The supernatant was discarded and cells were resuspended in a small amount of medium. Afterwards, cells were counted (cellomoterTM Auto T4; Nexcelom Bioscience), resuspended and plated (cell-culture plates CellstartR, Greiner bio-one) at the desired densities needed for the individual experiments. Additional harvests were performed on day 16, 21 and 23.

The purity of microglia preparations was routinely $>98 \%$, based on nuclear staining (DAPI), immunocytochemistry for CD11b, Iba1 and Griffonia simplicifolia isolectin B4 (Regen et al., 2011; Scheffel et al., 2012). 


\subsection{L929 fibroblast cultures}

To stimulate microglial proliferation in culture, cells were provided with supernatant from L929 fibroblasts as described by Regen et al. 2011. Fibroblasts were cultured in medium (DMEM containing 10\% FCS; $100 \mathrm{U} / \mathrm{ml}$ penicillin and $100 \mu \mathrm{g} / \mathrm{ml}$ streptomycin) and passaged (1:5) every 2 weeks. After 14 days of cultivation, the medium was collected and centrifuged at $1200 \mathrm{rpm}$ (290 g) for $10 \mathrm{~min}$ at $4^{\circ} \mathrm{C}$. Finally, the cell pellet was discarded and the supernatant was stored at $-20^{\circ} \mathrm{C}$ until usage. Fibroblasts were discarded after 30 passages and fresh L929 cultures were established.

\subsection{Cytokine and chemokine measurements}

The amount of secreted cytokines and chemokines by microglia was measured by using enzymelinked immunosorbent assay (ELISA). For ELISA, the DuoSet ELISA Development Kits (R\&D systems) were used for IL6 (interleukin 6) (\#DY406), MCP-1 (monocyte chemotactic protein 1) ( \#DY471), MIP1a (macrophage inflammatory protein 1a) (\#DY450), RANTES (regulated upon activation normal T-cell expressed and presumably secreted) (\#DY478) and KC (keratinocyte-derived chemokine) (\#DY453). For TNFa and for IL12-p40 (Interleukin 12), antibody pairs from BioLegend (\#430902) and eBioscience (\#88-7120-88) were used respectively.

In brief, microglia were plated in a 96-well plate with a density of 15000 cells/well. After incubation overnight at $37^{\circ} \mathrm{C}$ with $5 \% \mathrm{CO}_{2}$, cells were stimulated for 18 hours (if not stated differently) at $37^{\circ} \mathrm{C}$ with $5 \% \mathrm{CO}_{2}$. Subsequently, supernatants were transferred into new 96-well plates (Sarstedt; \#82.1581) and stored at $4^{\circ} \mathrm{C}$ or $-20^{\circ} \mathrm{C}$ for immediate or later processing, respectively. ELISA plates (Maxisorp; Nunc; \#456537) were coated with $50 \mu \mathrm{l} /$ well of the specific capture antibody solutions and incubated overnight at room temperature (RT) with a gentle agitation. Then, plates were washed 3 or 4 times (antibody dependent) with wash buffer (0.5\% Tween 20 in PBS) and blocked with $300 \mu \mathrm{l} /$ well of reagent diluent 1 (RD1) (1\% BSA in PBS) for $1 \mathrm{hr}$ at RT or overnight at $4^{\circ} \mathrm{C}$. Supernatants were diluted 1:10 (IL6; MCP-1; IL12-p40; RANTES; TNFa) or 1:20 (KC; MIP1a) in RD1 in dilution plates (Sarstedt; \#82.1582) and series of standard solutions provided by the kits were prepared. After discarding the block solution, plates were washed as mentioned above and 
$50 \mu \mathrm{l} /$ well of the diluted supernatants as well as the standards were added and incubated for 2 hours at RT or overnight at $4^{\circ} \mathrm{C}$. Next, plates were washed and $50 \mu \mathrm{l} /$ well of the detection antibody solution was added for $2 \mathrm{~h}$ at RT. After washing, wells received $50 \mu \mathrm{l}$ of a Streptavidin-HRP solution (R\&D; \#893975) for 20 minutes at RT protected from light. Following the next washing, $100 \mu \mathrm{l}$ substrate solution (TMB; Moss Inc. \#TMBUS-1000) was added and the color development was stopped by addition of $50 \mu \mathrm{l} /$ well of $1 \mathrm{~N} \mathrm{H}_{2} \mathrm{SO}_{4}$. Absorbance was measured at $450 \mathrm{~nm}$ with 540 $\mathrm{nm}$ reference by an iMark microplate reader (Bio-Rad). The amount of secreted cytokines and chemokines was calculated using a Microsoft Excel macro, specifically developed for this purpose by Dr. Jörg Scheffel and optimized at the department of neuropathology at the University Medical Center Göttingen.

\subsection{Cell viability assessment}

To determine any possible toxicity of the stimuli used for ELISA experiments on microglia, the cell viability test was performed routinely. To do so, microglia received fresh medium supplemented with $10 \%$ of water soluble tetrazolium salts (WST-1) reagent (Roche Applied Science) immediately after the transfer of the supernatant. The absorbance was quantified as a measure for viability after 3 hours of incubation at $37^{\circ} \mathrm{C}$ with $5 \% \mathrm{CO}_{2}$ using a microplate reader (iMark; BioRad) at $450 \mathrm{~nm}$ with $655 \mathrm{~nm}$ as the reference wavelength.

\subsection{Bromodeoxyuridine (BrdU) Cell Proliferation ELISA}

To assess microglia proliferation, the BrdU Cell Proliferation ELISA kit (Roche; \#11647229001) was used. Microglia were plated in a 96 well-plate with a density of 15000 cells/well and incubated for 24 hours at $37^{\circ} \mathrm{C}$ with $5 \% \mathrm{CO}_{2}$. Subsequently, the medium was replaced by various stimuli solutions including LPS (\#ALX-581-007, Alexis Biochemicals), $\mathrm{Pam}_{3} \mathrm{CSK}_{4}$ (\#165-066M002; Alexis Biochemicals) and Fibronectin (FN) (\#F-1141; Sigma) of different concentrations (0.01 ng/ml; $1 \mathrm{ng} / \mathrm{ml} ; 100 \mathrm{ng} / \mathrm{ml}$ for LPS and $\mathrm{Pam}_{3} \mathrm{CSK}_{4}, 25 \mu \mathrm{g} / \mathrm{ml} ; 50 \mu \mathrm{g} / \mathrm{ml} ; 100 \mu \mathrm{g} / \mathrm{ml}$ for FN) mixed with $10 \mu \mathrm{M}$ BrdU. Medium containing $10 \mu \mathrm{M}$ BrdU was used as control. Incubation for 6, 12, 24 or 48 hours followed by further processing as described by the BrdU Cell Proliferation 
ELISA assay. In brief, supernatants were removed and cells were fixed with $200 \mu \mathrm{l} /$ well FixDenat (provided by the assay) for 30 minutes at RT on a shaker. Next, supernatants were discarded and cells were incubated with $100 \mu \mathrm{l} /$ well anti-BrdU-POD working solution for 90 minutes at RT on a shaker. Cells were washed thrice with $200 \mu \mathrm{l} /$ well PBS and incubated with $100 \mu \mathrm{l} /$ well substrate solution for 5 minutes at RT. The reaction was stopped by addition of $25 \mu \mathrm{l} 2 \mathrm{~N} \mathrm{H}_{2} \mathrm{SO}_{4}$ for 1minute on a shaker. Absorbance was measured by an iMark microplate reader (Bio-Rad) at 450 $\mathrm{nm}$ (reference wavelength $690 \mathrm{~nm}$ or higher).

\subsection{Enzyme Linked Immuno Spot Assay (ELISpot)}

Production of TNFa and RANTES by individual microglia was quantified via Elispot.

For TNFa ELISpot experiments, the ELISpot assay against mouse TNFa (\# EL410; R\&D) or the TNFa/TNFSF1A ELISpot Development Module (R\&D; \#SEL410) were used. RANTES experiments were performed with the Mouse CCL5/RANTES ELISpot Development Module (R\&D; \#SEL478). Both development modules were combined with the ELISpot Blue Color Module (Strep-AP and BCIP-NBT) (R\&D \#SEL002) on Multiscreen HTS IP sterile plates (Millipore \#MSIPS4510).

The ELISpot plates were prepared as described by the manufacturer's instructions and a cell suspension mixed with different concentrations of LPS (Alexis Biochemicals; \#ALX-581-013), $\mathrm{Pam}_{3} \mathrm{CSK}_{4}$ (Alexis Biochemicals; \#165-066-M002) or MALP-2 (Macrophage-activating lipopeptide2) (Axxora/Apotech; \#APO-54N-018) was plated with a cell density of 1000, 2000 or 3000 cells/well. ELISpot plates were incubated at $37^{\circ} \mathrm{C}$ with $5 \% \mathrm{CO}_{2}$ for 24 hours and the development process was assessed according to the manufacturer's instructions. Spots were counted by the ELISpot reader ELI.Scan and analyzed using Eli.Analyse (both A.EL.VIS GmbH; Hannover). The obtained spot number was used for calculating the sizes of the subpopulations. The readouts for Spot intensities as well as the spot sizes were ranked in values between 1 to 17 and 1 to 10 , respectively. As controls, cells with the same density and stimulation conditions as used for the Elispot plate were plated on a separated plate (CellstartR, Greiner bio-one) simultaneously. This control plate was used for ELISA analysis of the cytokine secretion and viability tests. 


\subsection{Fluorescence-activated cell sorting (FACS)}

FACS was performed to quantify microglial subpopulations. For intracellular FACS staining of microglia, cells were plated at a density of 300000 cells/well in a 12-well plate unless otherwise stated. After an overnight incubation at $37^{\circ} \mathrm{C}$ with $5 \% \mathrm{CO}_{2}$ cells were stimulated with $0.01 \mathrm{ng} / \mathrm{ml}$; $0.1 \mathrm{ng} / \mathrm{ml} ; 1 \mathrm{ng} / \mathrm{ml} ; 10 \mathrm{ng} / \mathrm{ml} ; 100 \mathrm{ng} / \mathrm{ml}$ or $1000 \mathrm{ng} / \mathrm{ml}$ LPS (Alexis Biochemicals; \#ALX-581-013) for 8 hours.

The protein transport inhibitor Monensin (BioLegend, \#420701; 1:1000) was added after a stimulation period of $3 \mathrm{hr}$ (for RANTES staining or a RANTES/TNFa double staining) or $5 \mathrm{hr}$ (for TNFa staining) respectively. The stimulation was stopped by washing steps with medium and PBS. Cells were detached via incubation with $300 \mu \mathrm{l} 0.05 \%$ /0.02 \% Trypsin/EDTA (Biochrom; \#L2143) for 5 minutes at $37^{\circ} \mathrm{C}$. This reaction was stopped by adding $600 \mu \mathrm{l}$ medium. Cells were scraped carefully and transfered to $2 \mathrm{ml}$ Eppendorf tubes placed on ice. Subsequently, tubes were centrifugated at $800 \mathrm{~g}$ for $10 \mathrm{~min}$ at $4{ }^{\circ} \mathrm{C}$ and the supernatant was discarded. Cells were resuspended in $1 \mathrm{ml}$ FACS buffer (PBS containing 2\% FCS, $0.1 \%$ NaN3 and $0.01 \mathrm{M}$ EDTA pH 8.0) and centrifuged again. Supernatants were discarded and cells were incubated with $40 \mu \mathrm{l} \mathrm{Fc-Block} \mathrm{(BioLegend;}$ $\# 101310 ; 1: 100$ in FACS buffer) for $10 \mathrm{~min}$ at $4^{\circ} \mathrm{C}$ to prevent unspecific antibody binding. $40 \mu \mathrm{l}$ CD11b-Allophycocyanin (Cd11b-APC) (eBioscience; \#17-0112-83) or CD11b-Pacific Blue (CD11bPB) (BioLegend; \#101224) both diluted 1:100 in FACS buffer were added to the respective wells and incubated for $20 \mathrm{~min}$ at $4^{\circ} \mathrm{C}$ protected from light. Afterwards, cells were washed with 1 $\mathrm{ml} \mathrm{FACS}$ buffer and centrifuged at $800 \mathrm{~g}$ for $10 \mathrm{~min}$ at $4^{\circ} \mathrm{C}$. A resuspension of the cells in $200 \mu \mathrm{l}$ Cytofix/ Cytoperm (BD; \#51-2090KZ) was performed after discarding the supernatants. Cells were incubated for $20 \mathrm{~min}$ at $4^{\circ} \mathrm{C}$ protected from light, washed with $1 \mathrm{ml}$ saponin buffer $(0.1 \%$ Saponin (Sigma Aldrich; \#S-4521) in PBS) and centrifuged at $800 \mathrm{~g}$ for $10 \mathrm{~min}$ at $4^{\circ} \mathrm{C}$. Supernatants were removed and cells were incubated with $40 \mu \mathrm{l} \mathrm{Fc-Block} \mathrm{(1:100} \mathrm{in} \mathrm{saponin} \mathrm{buffer)} \mathrm{for} 5 \mathrm{~min}$ followed by addition of RANTES-Phycoerythrin (RANTES-PE) (BioLegend; \#149104), TNFa-PE (BioLegend; \#506305) or TNFa-Alexa Fluor 488 (BioLegend; \#506315), all $40 \mu \mathrm{l}, 1: 100$ in saponin buffer for 40 min at RT protected from light. Cells were washed with saponin buffer and centrifuged for at $800 \mathrm{~g}$ for $10 \mathrm{~min}$ at RT. Finally, cells were re-suspended in 170-200 $\mu \mathrm{l}$ FACS buffer and analyzed via the FACS Canto II (BD) and Flowjo (Tree Star, V10) software. For FACS analysis only Cd11b 
positive events were included.

For Zombie Aqua staining, cells were treated as mentioned above until the first wash step which was performed with PBS instead of FACS buffer. Cells were incubated with Zombie aqua (1:200 in PBS) for $10 \mathrm{~min}$ at RT protected from light followed by addition of the Fc-Block (1:100) and the CD11b (1:100) antibody.

To stain the proliferating cells, the Click-iT ${ }^{\circledR}$ EdU (5-ethynyl-2'-deoxyuridine) Alexa Fluor ${ }^{\circledR} 647$ Flow Cytometry Assay Kit (ThermoFischer SCIENTIFIC \#c-10419) was used. Cells were stimulated with $1 \mathrm{ng} / \mathrm{ml}$ LPS (Alexis Biochemicals; \#ALX-581-013) in combination with $5 \mu \mathrm{M}$ EdU (provided by the Kit) and harvested as mentioned before. Afterwards, cells were washed in $1 \mathrm{ml}$ of $1 \%$ BSA in PBS and incubated with Fc-Block and CD11b-PB prepared in 1\% BSA in PBS as well. Fixation, permeabilization, and EdU staining were performed as described by the proliferation kit. Intracellular antibodies were stained afterwards in Click-iT saponin based permeabilization and wash reagent instead of FACS buffer.

\subsection{Supernatant transfer experiment}

For the supernatant transfer experiment 200000 or 5000000 microglia cells were plated in a 12well plate and incubated over night at $37^{\circ} \mathrm{C}$ with $5 \% \mathrm{CO}_{2}$. The next day, wells received either fresh medium or LPS ( 1 or $10 \mathrm{ng} / \mathrm{ml}$ ) for 8 hours. Afterwards supernatants were transferred to wells containing 200000 unstimulated microglia. Microglia which received supernatant from LPS stimulated cells were left untouched for 8 hours. Microglia which received supernatant from unstimulated cells were stimulated with 1 or $10 \mathrm{ng} / \mathrm{ml}$ LPS for 8 hours.

This paradigm generated 3 different treatment groups. The first, the control group, consisted of 200000 unstimulated cells and 200000 cells that received the supernatant of 500000 unstimulated cells for 8 hours. The second group consisted of 200000 LPS-stimulated (1ng/ml) cells or 200000 cells with the supernatant of 500000 unstimulated cells that were stimulated after the supernatant transfer with $1 \mathrm{ng} / \mathrm{ml}$ LPS for 8 hours. The third group was made up by 200000 cells, treated for 8 hours with supernatants of 200000 or 500000 cells that were already stimulated with $1 \mathrm{ng} / \mathrm{ml}$ LPS for 8 hours beforehand. 
During the last incubation step microglia were supplemented with monensin for a subsepquent FACS analysis for TNFa and RANTES as previosly described.

\subsection{Insert experiment}

To evaluate the effect of the supernatant secreted by increasing numbers of microglia on microglia subpopulation sizes, an insert experiment was performed. Therefore, microglia were plated at a density of 200000 cells in a 12-well plate. Some of these wells received transmembrane cell-culture inserts (greiner bio-one


\#665640) containing addditional 100000, 200000 or 300000 microglia. After an overnight incubation at $37^{\circ} \mathrm{C}$ with $5 \% \mathrm{CO}_{2}$, microglia were stimulated with $1 \mathrm{ng} / \mathrm{ml}$ LPS for 8 hours. After the first three hours monensin was added to prevent cytokine secretion and after additional 5 hours microglia were further processed for FACS analysis of TNFa.

\subsection{Ribonucleic acid (RNA) extraction}

To extract RNA the RNeasy Mini Kit (Qiagen; \# 74104) was used. 800000 microglia/well were plated in a 6-well plate and stored at $37^{\circ} \mathrm{C}$ with $5 \% \mathrm{CO}_{2}$. Next, microglia were incubated with $10 \mathrm{ng} / \mathrm{ml} \mathrm{TNFa}$ for 1 or 12 hours at $37^{\circ} \mathrm{C}$ with $5 \% \mathrm{CO}_{2}$. Supernatants were discarded, cells were washed and incubated with $1 \mathrm{ng} / \mathrm{ml}$ LPS for 3 hours at $37^{\circ} \mathrm{C}$ with $5 \% \mathrm{CO}_{2}$. Afterwards, cells were washed with pre-warmed PBS and received $350 \mu$ RLT buffer (if not stated otherwise provided by the kit) containing $1 \% \beta$-Mercaptoethanol. Cells were scraped carefully, the lysates were transferred into Eppendorf-tubes and after resuspension into QIAshredder Mini Spin Columns (Qiagen; \#1011711). The columns were centrifuged at maximum speed for 2 minutes and $350 \mu \mathrm{l}$ 
$70 \% \mathrm{EtOH}$ was added to the lysate containing collection tubes. Lysates were transferred after resuspension to RNeasy Mini Spin Columns (Qiagen; \#1011708) and centrifuged for $30 \mathrm{sec}$ at 8000 g. Collection tubes were emptied, $350 \mu \mathrm{l}$ RW1 buffer was added on top of the spin columns and a centrifugation at $8000 \mathrm{~g}$ for $30 \mathrm{sec}$ followed. Then, $75 \mu \mathrm{l}$ DNase mix $(8,75 \mu \mathrm{l}$ DNase $+61,25 \mu \mathrm{l}$ RD buffer) was added on top of the spin column membranes and incubated for 15 minutes at RT. Subsequently, $250 \mu \mathrm{l}$ RW1 buffer was added and the column was centrifuged for $30 \mathrm{sec}$ at 8000 g. Collection tubes were emptied, $500 \mu \mathrm{l}$ RPE buffer was added and the column was centrifuges as before. Next, RPE buffer was added again and centrifuged for $3 \mathrm{~min}$ at $8000 \mathrm{~g}$. The collection tube was replaced by a new one and the columns were centrifuged for $1 \mathrm{~min}$ at maximum speed. Finally, columns were placed in Eppendorf tubes, $25 \mu \mathrm{l}$ nuclease-free water was added and centrifuged for $1 \mathrm{~min}$ at $8000 \mathrm{~g}$. This step was repeated one more time and the obtained RNA concentration was measured using the NanoDrop ND-1000 Spectrophotometer (PEQLAB) and the corresponding software version 3.8.1. Samples were stored at $-80^{\circ} \mathrm{C}$ until further processing.

\subsection{Complementary deoxyribonucleic acid (cDNA) synthesis}

For quantifying changes in gene expression upon stimulation, quantitative Real-Time Polymerase chain reaction (RT-PCR) was performed. cDNA for RT-PCR was synthesized by using the QuantiTect Reverse Transcription Kit (Qiagen; \#205311) as followed: the template RNA was thawed on ice and the genomic DNA (Deoxyribonucleic acid) elimination cocktail was prepared. The composition was RNA concentration dependent and contained $300 \mathrm{ng}$ RNA, $2 \mu \mathrm{l}$ wipeout buffer and RNase- free water to make a total volume of $15 \mu$ l. This mixture was incubated for 2 minutes at $42^{\circ} \mathrm{C}$ and immediately placed on ice afterwards. Next, the reverse transcription master mix containing $1 \mu \mathrm{l}$ Quantiscript Reverse Transcriptase, $4 \mu \mathrm{l}$ Quantiscript RT buffer (5x) and $1 \mu \mathrm{l}$ RT Primer mix per sample was prepared. $14 \mu \mathrm{l}$ of the DNA elimination cocktail was mixed with the reverse transcription master mix and incubation at $42^{\circ} \mathrm{C}$ for 15 minutes and $95^{\circ} \mathrm{C}$ for 3 minutes followed. Finally, $100 \mu \mathrm{l}$ RNase-free water was added to each sample (1:6 dilution) and samples were transferred to a 96-well plate and stored at $-20^{\circ} \mathrm{C}$ until further processing by real-time PCR. 


\subsection{RT-PCR}

For RT-PCR iTaq universal SYBR Green supermix (BIO-RAD; \#172-5121) and cDNA was thawed at RT. For each desired sample, $10 \mu \mathrm{l}$ iTaq universal SYBR Green supermix, $1 \mu \mathrm{l}$ forward and reverse primer (see table 2.1) and $4 \mu \mathrm{l} \mathrm{H} 2 \mathrm{O}$ was mixed. $15 \mu \mathrm{l}$ of this mixture was added in triplicates to a qPCR plate and $5 \mu \mathrm{l}$ of the appropriate cDNA was added to the mixture. The plate was sealed with an optically transparent sealing tape (Sarstedt; \#95.1994) and vortexed for $30 \mathrm{~s}$. Next, the plate was centrifuged at maximum speed for 5 minutes to remove air bubbles within the wells. The plate was inserted into the real-time cycler QuantStudio 7 Flex (life technologies) and processed as described (see table 2.2). Results were analyzed using the QuantStudio Realtime PCR Software (version 1.1; life technologies). Relative mRNA expression for MCP-1, MIP1a, RANTES, KC and TNFa was calculated by $2^{-\Delta \Delta C T}$ to medium controls with Gapdh as the control gene.

Table 2.1 - RT-PCR primer

\begin{tabular}{|c|c|c|}
\hline Name & Company & Number \\
\hline PrimePCR ${ }^{\mathrm{TM}}$ SYBR $^{\circledR}{ }^{\circledR}$ Green Assay: TNF, Mouse & BIO-RAD & qMmuCED0004141 \\
\hline PrimePCR $^{\mathrm{TM}}$ SYBR $^{\circledR}$ Green Assay: MIP1a, CCL3, Mouse & BIO-RAD & qMmuCED0003870 \\
\hline PrimePCR $^{\mathrm{TM}}$ SYBR $^{\circledR}$ Green Assay: RANTES, CCL5, Mouse & BIO-RAD & qMmuCID0021047 \\
\hline PrimePCR ${ }^{\mathrm{TM}}$ SYBR $^{\circledR}$ Green Assay: Gapdh, Mouse & BIO-RAD & qMmuCED0027497 \\
\hline PrimePCR ${ }^{\mathrm{TM}}$ SYBR $^{\circledR}$ Green Assay: KC, CXCL1, Mouse & BIO-RAD & qMmuCED0003898 \\
\hline
\end{tabular}

Table 2.2 - RT-PCR cycler conditions

\begin{tabular}{|c|c|c|l|}
\hline Cycles & Duration & Temperature & Step \\
\hline 1 & $2 \mathrm{~min}$ & $95^{\circ} \mathrm{C}$ & Activation \\
\hline 40 & $15 \mathrm{sec}$ & $95^{\circ} \mathrm{C}$ & Denaturation \\
\hline & $45 \mathrm{sec}$ & $60^{\circ} \mathrm{C}$ & Anneling/extension \\
\hline 1 & $1 \mathrm{~min}$ & $95^{\circ} \mathrm{C}$ & \\
\hline 1 & $1 \mathrm{~min}$ & $55^{\circ} \mathrm{C}$ & Melt curve \\
\hline 80 & $10 \mathrm{sec}$ & $55-94.5^{\circ} \mathrm{C}$ & increase set point temperature after cycle 2 by $0.5^{\circ} \mathrm{C}$ \\
\hline & hold & $4^{\circ} \mathrm{C}$ & \\
\hline
\end{tabular}




\subsection{Lactate dehydrogenase (LDH) assay}

The LDH Activity Assay kit (Sigma-Aldrich; \#MAK066) was used to assess the presence of damage or toxicity in microglia cells. The assay was performed according to the manufacturer's instructions. In brief, samples (microglia supernatants, taken after stimulation) were diluted 1:10 with LDH Assay buffer and the NADH standards were prepared in a 96-well plate with a volume of $50 \mu \mathrm{l} /$ sample or standard. Next, $50 \mu \mathrm{l}$ of the master reaction mix composed of $48 \mu \mathrm{l}$ LDH Assay Buffer and $2 \mu \mathrm{l}$ LDH Substrate Mix, was added on top and the plate incubated at RT on a horizontal shaker. After 2 minutes, the optical absorbance was measured at $450 \mathrm{~nm}$ by an iMark microplate reader (Bio-Rad) every five minutes until the value of the most active sample exceeded the value of the highest standard. Finally, the LDH activity was calculated as described by the manufacturer's instructions.

\subsection{Statistical analysis}

Data were presented as mean \pm SEM and analysed by GraphPad Prism ${ }^{\circledR} 6.01$ (Graphpad Software). Data sets were analyzed via the Mann-Whitney test, multiple t-test or ANOVA and Tukey's multiple comparison. Differences of $\mathrm{p}<0.05$ were considered as significant. 


\section{Results}

\subsection{Subpopulation sizes are dependent on the severity of the challenge}

The amount of cytokines like TNFa, released by microglia, correlates well with the severity of the challenge that they face, while only subsets of microglia are producing this respective cytokine (Regen et al., 2011; Scheffel et al., 2012). However, the principle of organization behind this phenomenon is still unknown. Here, I investigated the functional link between cytokine release and subpopulation size as well as a relative cytokine release per cell. Hence, I performed ELISpot and ELISA experiments for TNFa and RANTES on 1000 microglia cells mixed with increasing concentrations (0.01ng/ml; $0.1 \mathrm{ng} / \mathrm{ml} ; 1 \mathrm{ng} / \mathrm{ml}, 10 \mathrm{ng} / \mathrm{ml}, 100 \mathrm{ng} / \mathrm{ml}$ and $1000 \mathrm{ng} / \mathrm{ml})$ of LPS, $\mathrm{Pam}_{3} \mathrm{CSK}_{4}$ and MALP-2. The combination of both quantitative techniques allowed a correlation analysis between the cytokine secreting microglia number and the corresponding amount of secreted cytokines.

Evaluation of the ELISpot experiments for both TNFa and RANTES revealed that the cytokine secreting subpopulation size and the cytokine secretion increased signifcantly with increasing stimuli concentrations. At a certain stimuli concentration, the stimuli were not able to induce a further increase which resulted in a ceilling effect for both measured values (see figure 3.1A, p.25).

The microglia subpopulations which produced TNFa upon MALP-2 stimulation range from 41.3 $\%$ to $77.9 \%$ with a cytokine production of up to $372.6 \mathrm{pg} / \mathrm{ml}$. Stimulation with $\mathrm{Pam}_{3} \mathrm{CSK}_{4}$ showed an increase in the subpopulation size from about $32.5 \%$ to $79.1 \%$, while the total produced amount of TNFa rose to a maximum of $688.9 \mathrm{pg} / \mathrm{ml}$. LPS stimulation increased the microglia subpopulations from $45.1 \%$ to about $87.0 \%$ with a total production of up to $924.7 \mathrm{pg} / \mathrm{ml}$. The increases in the TNFa-producing subpopulations as well as the TNFa production reached a plateau at concentrations above $0.1 \mathrm{ng} / \mathrm{ml}$ for MALP-2 and LPS, and $10 \mathrm{ng} / \mathrm{ml}$ for $\mathrm{Pam}_{3} \mathrm{CSK}_{4}$ stimulation. 
A MALP-2 stimulation $\mathrm{Pam}_{3} \mathrm{CSK}_{4}$ stimulation LPS stimulation
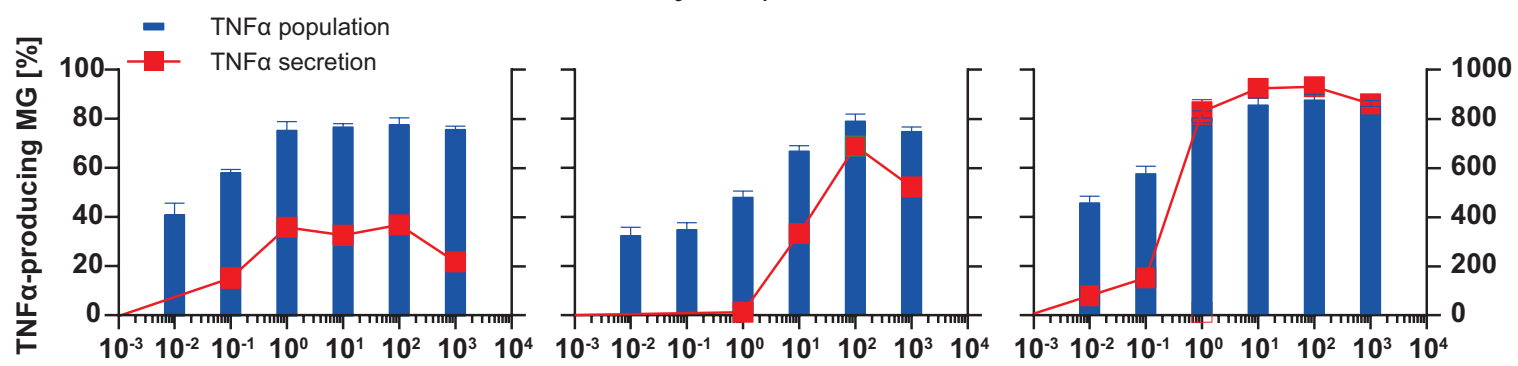

-1
2
0
0
0
0
0
0
0
0
0
0
$\overline{0}$
0
0
3
3

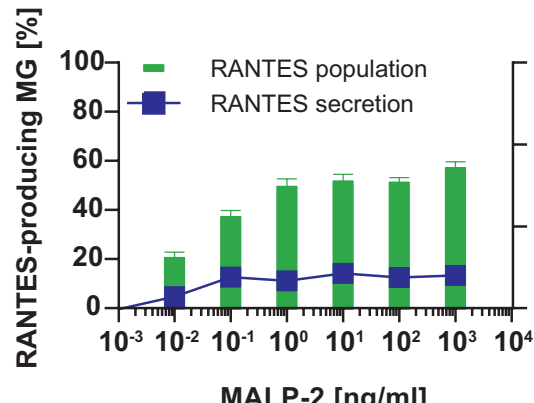

MALP-2 [ng/ml]




B

\section{C}

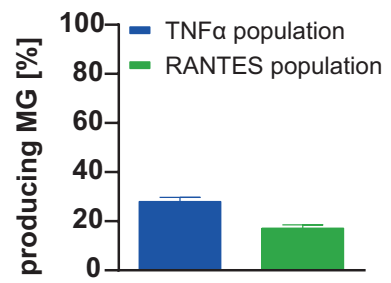

unstimulated cells



TNFa-producing MG [\%]

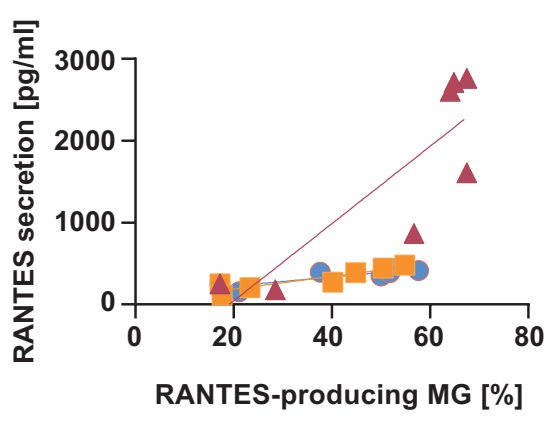

Figure 3.1 - ELISpot, ELISA and correlation analysis of TNFa and RANTES-producing microglia subpopulations: A) TNFa and RANTES producing subpopulation sizes obtained by ELISpot experiments for microglia plated at a density of 1000 cells/well. Microglia were stimulated with the indicated concentrations of LPS, $\mathrm{Pam}_{3} \mathrm{CSK}_{4}$ or MALP-2 for 24 hours (bars) and the corresponding amount of the secreted cytokines (lines) was obtained by ELISA. The stimuli dependent growth is significantly different for all tested conditions (ANOVA with $\mathrm{P}<0.0001$ for all subpopulations and TNFa secretions. RANTES secretion has $\mathrm{P}$ values of $\mathrm{P}_{M A L P-2}=0.0002, \mathrm{P}_{\text {Pam } 3 C S K 4}=0.0095$ and $\left.\mathrm{P}_{L P S}<0.0001\right)$. B) Corresponding TNFa and RANTES producing subpopulation sizes of unstimulated microglia. C) Stimuli-dependent correlation of the cytokine secretion vs. the associated subpopulation size for TNFa and RANTES analysed with pearson correlation. Data are presented mean \pm SEM. $n=8-16$ 
The microglia subpopulations positive for RANTES showed a similar pattern. MALP-2 stimulation led to an increase from $21.1 \%$ to about $57.6 \%$ with a maximum production of $440.9 \mathrm{pg} / \mathrm{ml}$. Stimulation with $\mathrm{Pam}_{3} \mathrm{CSK}_{4}$ showed an increase from about $17.7 \%$ to $54.8 \%$ and a total cytokine production of up to $480.2 \mathrm{pg} / \mathrm{ml}$. Stimulation with LPS increased the subpopulations from $28.4 \%$ to 67.5 and the RANTES secretion to $2763.2 \mathrm{pg} / \mathrm{ml}$. The plateau reached by the RANTESproducing subpopulations appeared at similar concentrations observed for the TNFa-producing subsets, while the cytokine secretion stagnates at concentrations above $0.1 \mathrm{ng} / \mathrm{ml}$ for MALP-2 and $10 \mathrm{ng} / \mathrm{ml}$ for $\mathrm{Pam}_{3} \mathrm{CSK}_{4}$ and LPS stimulation. The obtained results also revealed that a population of TNFa and RANTES producing microglia is already existing even if the cells were not exposed to a challenge (see figure 3.1B, p.25). While the subsets comprised $28.1 \%$ of all microglia for TNFa and $17.3 \%$ for RANTES, measurable cytokine secretion could not be demonstrated in the unstimulated cells.

The increase in both, subpopulation size and cytokine secretion highly correlates for all tested stimuli regarding TNFa, with $\mathrm{R}^{2}$ values above 0.9 (see figure 3.1C, p.25) (see table 3.1). A weaker, though still considerable correlation was found regarding RANTES with $\mathrm{R}^{2}$ values of 0.72 for MALP-2, 0.85 for $\mathrm{Pam}_{3} \mathrm{CSK}_{4}$ and 0.74 for LPS stimulation.

To validate the findings from ELISpot, FACS analysis was performed on microglia stimulated with the same concentrations of LPS. Thereby, I was able to confirm the concentration dependent cytokine producing subpopulation sizes for Table $3.1-\mathrm{R}^{2}$ of cytokine producing subpopulation sizes and cytokine secretion (obtained by pearson correlation)

\begin{tabular}{|l|c|c|}
\hline & TNFa & RANTES \\
\hline MALP-2 & 0.902 & 0.740 \\
\hline Pam $_{3}$ CSK $_{4}$ & 0.917 & 0.845 \\
\hline LPS & 0.938 & 0.720 \\
\hline
\end{tabular}
both, TNFa and RANTES (see figure 3.2, p.27). The subpopulations for TNFa-secreting microglia increased from $4.6 \%$ at $0.01 \mathrm{ng} / \mathrm{ml}$ to $59.5 \%$ while RANTESsecretion subpopulations increased from $4.7 \%$ to $50.3 \%$. The significant increase in both, TNFaand RANTES-producing subpopulations reached a plateau at LPS concentrations above $0.1 \mathrm{ng} / \mathrm{ml}$. A second factor besides subpopulation size that contributes to the overall cytokine release is the cytokine release per cell (see figure 3.3, p.27). TNFa secretion per cell increased significantly between MALP-2 concentrations of $0.01 \mathrm{ng} / \mathrm{ml}, 0.1 \mathrm{ng} / \mathrm{ml}$ and $1 \mathrm{ng} / \mathrm{ml}$ and stagnated at higher concentrations. LPS-stimulated microglia behaved similarly with significant increases between 


\section{TNFa-producing subpopulation size}

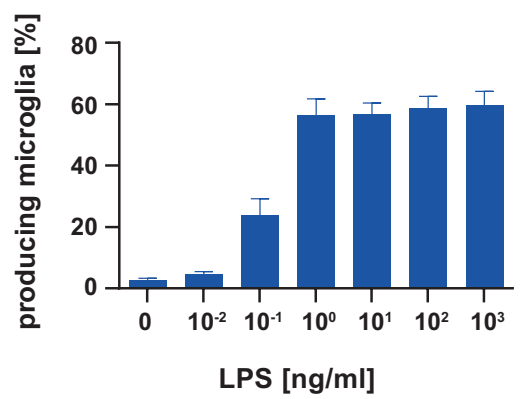

RANTES-producing subpopulation size



Figure 3.2 - FACS analysis of TNFa and RANTES-producing microglia subpopulations: FACS of microglia plated at a density of 300000 cells/well and stimulated with LPS for 8 hours was investigated for TNFa and RANTES producing subpopulation sizes. Subpopulation size progression is significant with $\mathrm{P}<0.0001$ for TNFa and RANTES (ANOVA). Data are presented as mean \pm SEM. $n=6$

\section{Cytokine secretion per secreting cell}
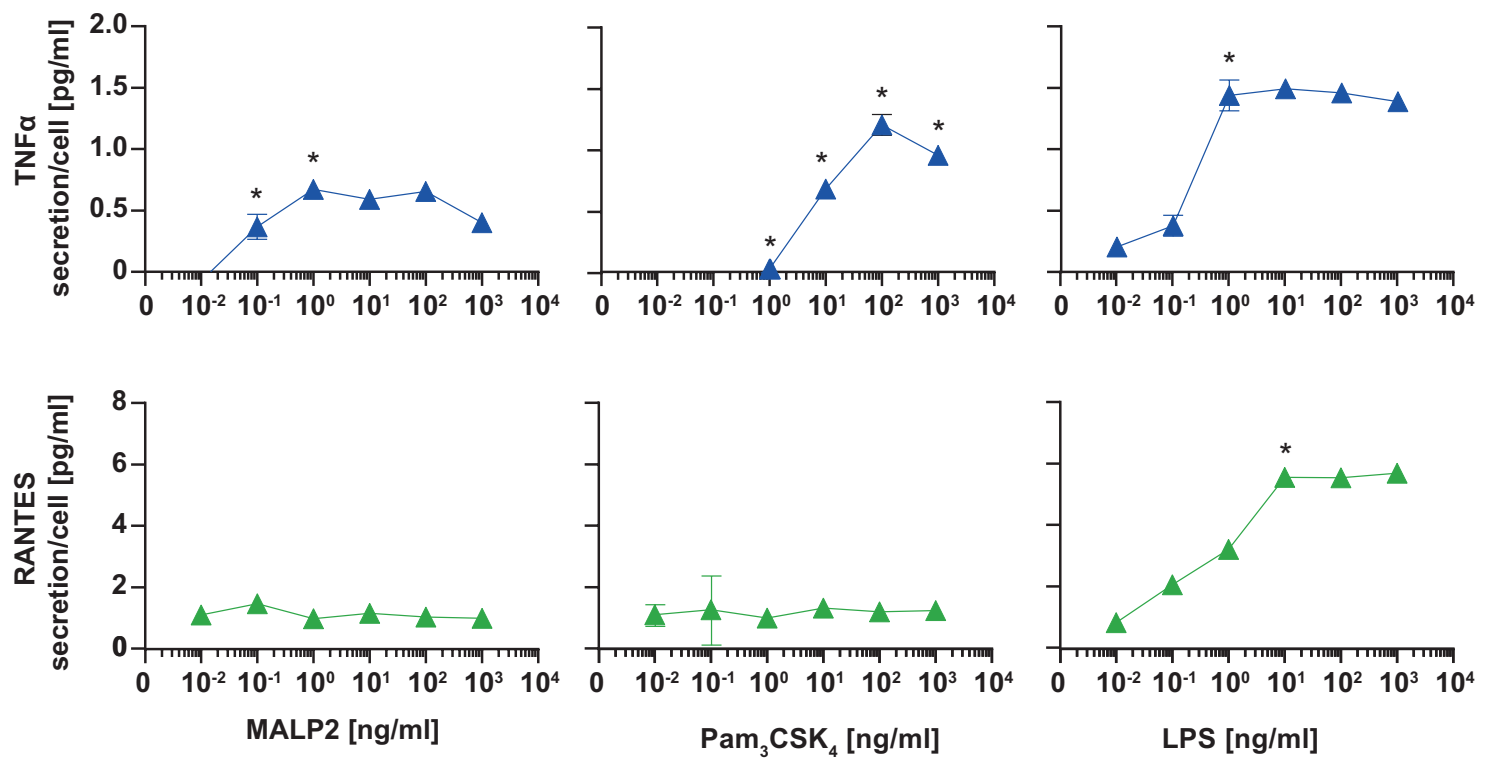

Figure 3.3 - Amount of cytokine secretion per cell of TNFa- and RANTES-producing microglia subpopulations. Asterisks indicate significant differences between the concentration and the next lower one. Significance was tested via ANOVA and Tukey's multiple comparison (ANOVA: $\mathrm{P}<0.0001$ for all TNFa and the RANTES LPS group).Data are presented as mean \pm SEM. $n=8-16$ )

0.1 and $1 \mathrm{ng} / \mathrm{ml}$ and a stabilization afterwards. Pam ${ }_{3} \mathrm{CSK}_{4}$ showed a rather dynamic progression with a gradual increase of secretion per cell and a peak around $100 \mathrm{ng} / \mathrm{ml}$. No change of the cytokine secretion per cell could be observed after stimulation with MALP-2 and $\mathrm{Pam}_{3} \mathrm{CSK}_{4}$ 
regarding RANTES. LPS showed a significant increase from $1 \mathrm{ng} / \mathrm{ml}$ to $10 \mathrm{ng} / \mathrm{ml}$ and a stable secretion level at higher concentrations.

A
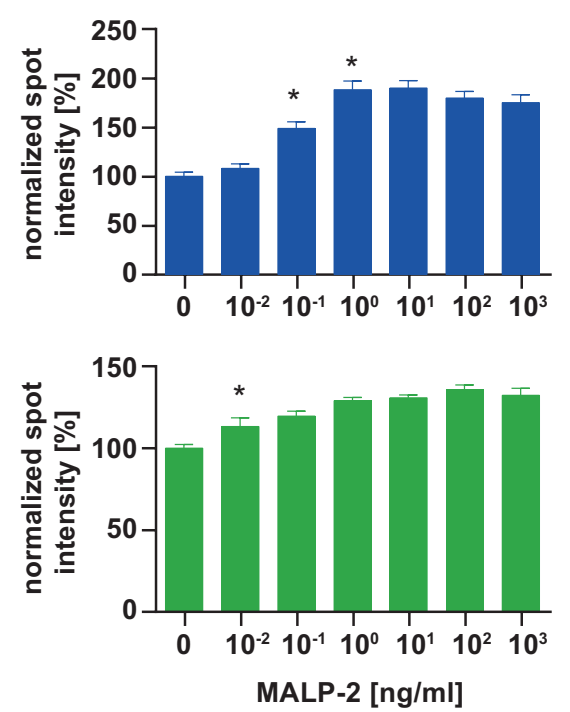

B
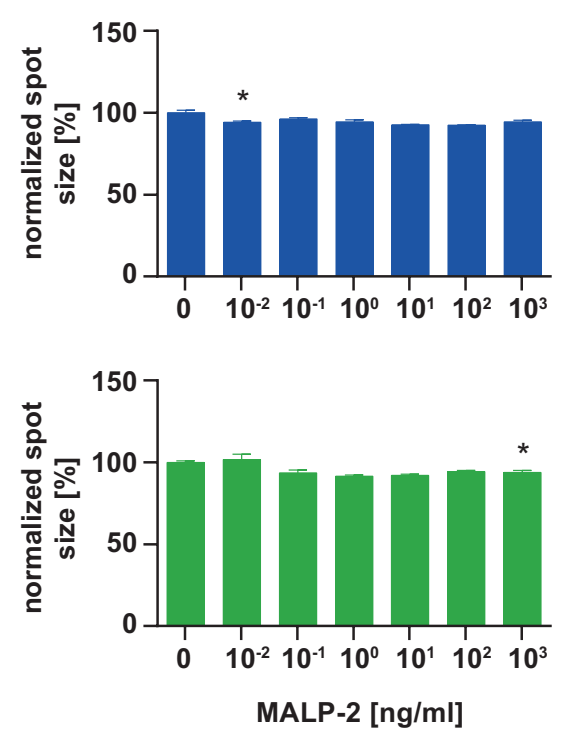

\section{ELISpot spot intensity}


ELISpot spot size
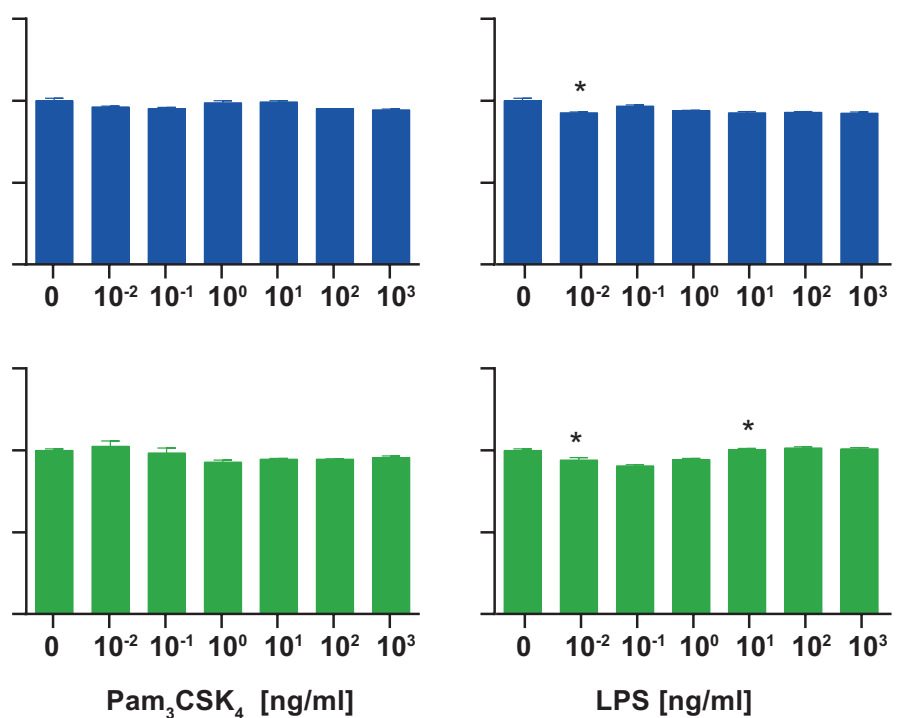
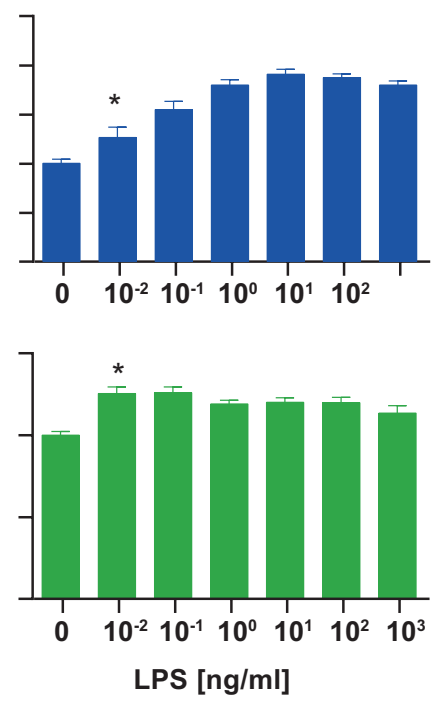

$10^{-2} 10^{-1} 10^{0} 10^{1} 10^{2} 10^{3}$

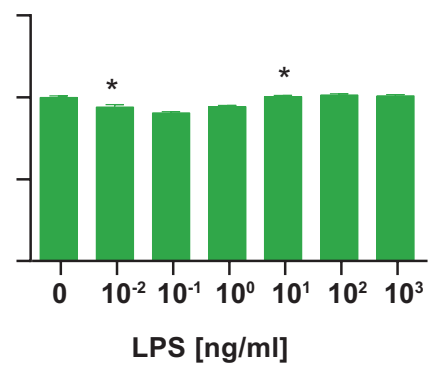

Figure 3.4 - Analysis of Spot intensity and Spot size of TNFa- and RANTES-producing microglia subpopulations: A) ELISpot intensities of the TNFa and RANTES spots normalized to medium control. B) ELISpot spot sizes for TNFa and RANTES normalized to medium control; $n=6$. Significance was tested via ANOVA and Tukey's multiple comparison. Asterisks indicate significant differences between the concentration and the next lower one with a P-value $\leq 0.05$. Data are presented as mean \pm SEM. $n=8-16$ 
The relationship between subpopulation size and secretion per cell could be validated by ELISpot as well. An elevation of the secreted amount could be reflected by an increase in spot size and/or spot intensity. Analysis of these factors revealed an increase in the TNFa ELISpot spot intensity for all stimuli and in the spot size for MALP-2 and LPS stimulation. The RANTES ELISPOT intensities as well as spot sizes grew significantly by MALP-2 and LPS stimulation. No effect of $\mathrm{Pam}_{3} \mathrm{CSK}_{4}$ could be detected considering RANTES (see figure 3.4, p.28). The TNFa spot intensity increased significantly between $0.01 \mathrm{ng} / \mathrm{ml}, 0.1 \mathrm{ng} / \mathrm{ml}$ and $1 \mathrm{ng} / \mathrm{ml}$ of MALP-2-stimulated microglia. $\mathrm{Pam}_{3} \mathrm{CSK}_{4}$ led to significant increases between $0.1 \mathrm{ng} / \mathrm{ml}, 1 \mathrm{ng} / \mathrm{ml}$ and $10 \mathrm{ng} / \mathrm{ml}$ and LPS between medium control and 0.01 ng/ml. RANTES-producing microglia stimulated with MALP-2 and LPS showed no significant differences in the spot intensity of $0.01 \mathrm{ng} / \mathrm{ml}$ compared to medium

\section{Combined stimulation}



Figure 3.5 - ELISpot analysis of TNFa-producing microglia populations after combined stimulation: TNFa and RANTES producing subpopulation sizes obtained by ELISpot experiments for microglia plated at a density of 1000 cells/well, stimulated with the indicated concentrations of LPS, $\mathrm{Pam}_{3} \mathrm{CSK}_{4}$, MALP-2or combinations of them for 24 hours; $n=8$. Asterisks indicate significant differences of LPS and MALP-2 or $\mathrm{Pam}_{3} \mathrm{CSK}_{4}$ combined stimulation to LPS only stimulation with p-values ${ }^{*} \leq 0.05 ;{ }^{* *} \leq 0.005 ;{ }^{* * *} \leq 0.0005 \mathrm{ob}$ tained by multiple t-test.Data are presented as mean \pm SEM. $\mathrm{n}=8$ )

only, while $\mathrm{Pam}_{3} \mathrm{CSK}_{4}$ could not elicit an increase in the spot intensities.

Analysis regarding ELISpot spot size revealed sparse differences only (see figure 3.4B, p.28). Significant differences for TNFa were detected for $0.01 \mathrm{ng} / \mathrm{ml}$ of MALP-2 and LPS stimulated microglia compared to medium control. RANTES-producing microglia stimulated with MALP2 showed significant differences between $100 \mathrm{ng} / \mathrm{ml}$ and $1000 \mathrm{ng} / \mathrm{ml}$ while the spot sizes of LPS 
stimulated cells differed between medium and $0.01 \mathrm{ng} / \mathrm{ml}$ as well as $1 \mathrm{ng} / \mathrm{ml}$ and $10 \mathrm{ng} / \mathrm{ml}$. No differences could be observed in $\mathrm{Pam}_{3} \mathrm{CSK}_{4}$ stimulated microglia, neither for TNFa nor for RANTES. Next, a similar experiment with parallel stimuli was performed to investigate whether simultaneous stimulation has an additive effect on TNFa producing microglia subpopulations. Combinations of LPS and Pam ${ }_{3} \mathrm{CSK}_{4}$, LPS and MALP-2 as well as Pam ${ }_{3} \mathrm{CSK}_{4}$ and MALP-2 at concentration of $0.01 \mathrm{ng} / \mathrm{ml}, 0,1 \mathrm{ng} / \mathrm{ml}$ and $1 \mathrm{ng} / \mathrm{ml}$ were used for this experimental approach. However, no additive effect on the subpopulation sizes could be observed (see figure 3.5, p.29). The combination of LPS with another stimuli in most cases even lead to a slight reduction of the TNFa producing subpopulation size than a LPS only stimulation. Combination of $\mathrm{Pam}_{3} \mathrm{CSK}_{4}$ and MALP-2 showed no effect at all.

In summary, I could demonstrate a stimuli-dependent increase in TNFa and RANTES-producing microglia subpopulations via ELISpot and FACS. The subpopulations do not rise infinetly but reach a plateau at higher stimuli concentrations. The maximal subpopulation sizes could not be raised any further by a combination of different stimuli. The increase in the subpopulation sizes correlates highly with an increase in the cytokine secretion, which is more pronounced for TNFa than RANTES in stimulated microglia. An increase in the cytokine secretion per cell could also be demonstrated at higher stimuli concentrations, despite this correlation. This increase was primarily demonstrated in TNFa producing subsets, but also in LPS stimulated RANTES subpopulations. The increase in the cytokine secretion per cell correlates with an increase in the intensity of the spots obtained by the ELISpot experiments, which validates this observation even further. 


\subsection{Microglial subpopulation sizes are density dependent}

Microglia are heterogeneously distributed throughout the brain, with densities reaching region dependently from 5 to $12 \%$ (Lawson et al., 1990). To test whether microglia densities affect the specific subpopulation sizes, I performed ELISpot measurements for TNFa and RANTES in a similar approach as previously described, though, with higher cell densities (2000 and 3000 cells/well). The high RANTES production exceeded the detection limit, thus a precise spot discrimination was impossible (data not shown). Data from the TNFa ELISpot experiment revealed

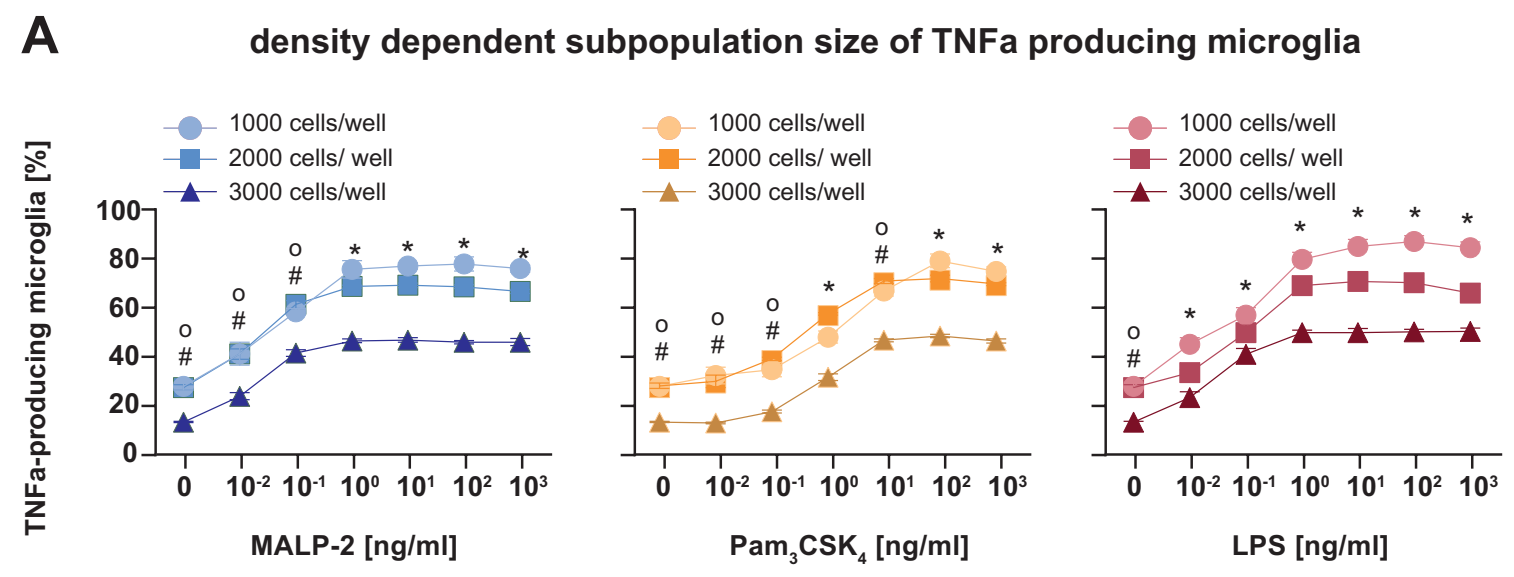

B

MALP-2 stimulation

$\mathrm{Pam}_{3} \mathrm{CSK}_{4}$ stimulation

LPS stimulation
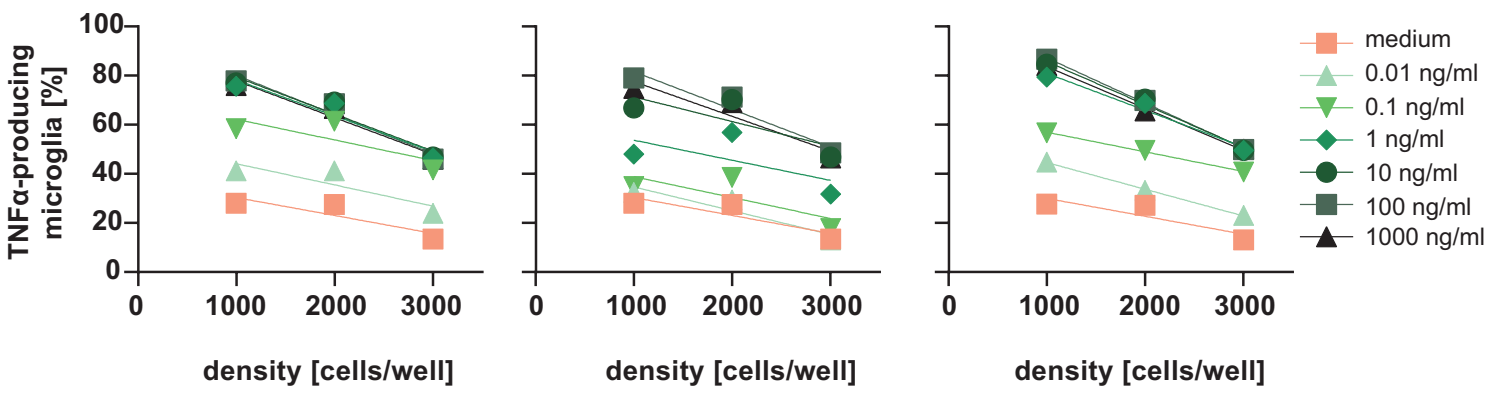

Figure 3.6 - Density dependency of microglia subpopulations analyzed by ELISpot: A) TNFa-producing microglia subpopulations obtained by ELISpot experiments with microglia densities of 1000, 2000 and 3000 cells/well. Significant differences obtained by ANOVA and Tukey's multiple comparison with a P-value $\leq$ 0.05 were indicated as followed: * difference between all densities; + differences between 1000 and 2000 cells/well; \# differences between 1000 and 3000 cells/well; o differences between 2000 and 3000 cells/well. B) Correlation between TNFa-producing subpopulation sizes and microglia density analyzed with pearson correlation. Data are presented as mean \pm SEM. $n=8$ 
Table 3.2 $-\mathrm{R}^{2}$ of TNFa producing subpopulation sizes vs. microglia density (obtained by pearson correlation)

\begin{tabular}{|l|c|c|c|c|c|c|c|}
\hline & medium & $\mathbf{0 . 0 1} \mathbf{~ n g} / \mathbf{m l}$ & $\mathbf{0 . 1} \mathbf{~ n g} / \mathbf{m l}$ & $\mathbf{1} \mathbf{~ n g} / \mathbf{m l}$ & $\mathbf{1 0} \mathbf{~ n g} / \mathbf{m l}$ & $\mathbf{1 0 0} \mathbf{~ n g} / \mathbf{m l}$ & $\mathbf{1 0 0 0} \mathbf{~ n g} / \mathbf{m l}$ \\
\hline MALP-2 & 0,774 & 0,7552 & 0,6189 & 0,9188 & 0,9290 & 0,9453 & 0,9536 \\
\hline Pam $_{3}$ CSK $_{4}$ & 0,7742 & 0,8684 & 0,5917 & 0,4120 & 0,6274 & 0,9270 & 0,8953 \\
\hline LPS & 0,7742 & 0,9992 & 0,9963 & 0,9744 & 0,9883 & 0,9973 & 0,9976 \\
\hline
\end{tabular}

that the TNFa-producing subpopulation sizes are negatively density-dependent as they increase with decreasing cell density (see figure 3.6, p.31). The previously observed concentration dependent increase in subpopulation size (see figure 3.1, p.25) was observed in densities of 2000 and 3000 cells/well as well, though, saturation was already reached at approximately $60 \%$ and $40 \%$ respectively. Significant differences between the densities could be demonstrated for all used stimuli. The differences could be either observed between 1000 and 2000 vs. 3000 cells, or between all densities which became more pronounced with higher stimuli concentrations (see figure 3.6A, p.31). Correlation analysis of the TNFa-producing microglia subpopulation size and the plated microglia density demonstrated a negative correlation for most conditions (see figure 3.6B, p.31) (see table 3.2). LPS stimulation resulted in $\mathrm{R}^{2}$ values above 0.97 for all conditions except medium $\left(\mathrm{R}^{2}=0.77\right)$. MALP-2 stimulation revealed $\mathrm{R}^{2}$ values of $0.774,0.7552$ and 0.6189 for medium control and the lower concentrations and $\mathrm{R}^{2}$ values above 0.91 for the higher ones. The $\mathrm{R}^{2}$ values for $\mathrm{Pam}_{3} \mathrm{CSK}_{4}$ were much more heterogeneous reaching from 0.41 up to 0.93 .

FACS experiments performed with increasing microglia densities (200000, 300000, 400000 and 500000 cells/well) for TNFa validated these results by showing a similar pattern (see figure 3.7A, p.33). The higher the density, the lower the secreting subpopulation size which declines at the highest density to about $82 \%$ of the lowest one. A similar correlation can be observed for RANTES positive microglia populations, since a significant difference could be demonstrated for 200000 and 300000 cells/well vs. 500000 cells/well; with a decrease to about $75 \%$ of the lowest density. To rule out a possible effect of increased cell death caused by the relatively high cell densities, I stained for Zombie, a marker for dead cells. As seen in figure 3.7B, no differences among the various cell densities could be measured, confirming that none of the used cell densities biased the observed phenomenon by increased cell death. 

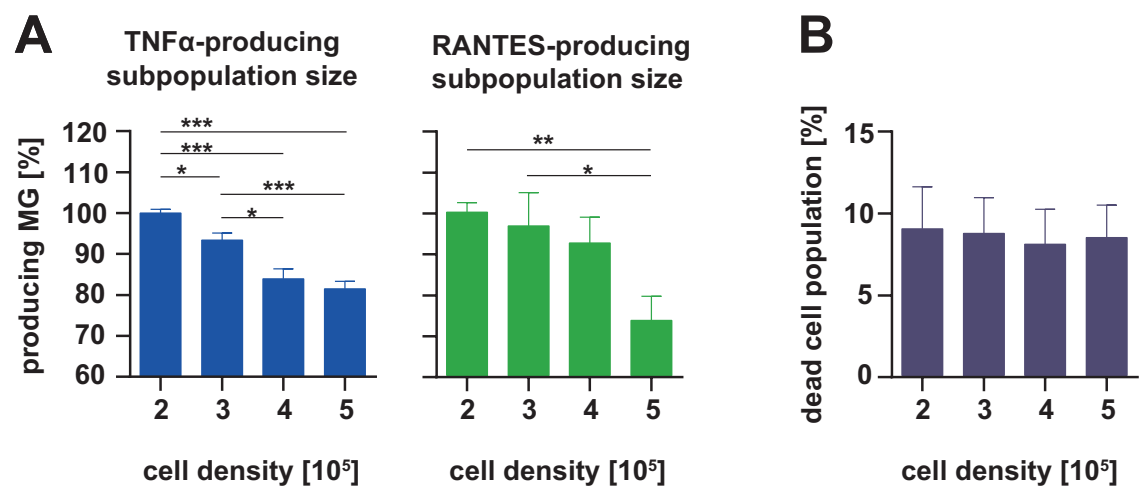

Figure 3.7 - Influence of the cell density on the microglia subpopulation sizes and cell death analyzed by FACS: FACS analysis of microglia plated at 200000, 300000, 400000 or 500000 cells/well. Microglia were stimulated with $1 \mathrm{ng} / \mathrm{ml}$ LPS for 8 hours and received monensin after 3 hours. Cells were screened for TNFa, RANTES (A) and Zombie (B) a marker for dead cells. Data was normalized to 200000 cells/well. Data was analyzed via Mann-Whitney test. Asterisks indicate p-values $* \leq 0.05 ;^{* *} \leq 0.005 ; * * * \leq 0.0005$. B) .Data are presented as mean \pm SEM. $n=6$

As I was able to confirm my hypothesis on density dependent subpopulation sizes of microglia, it was now of utmost interest to investigate the principle of organization. Thus, I investigated the progression of the subpopulation size increase for all densities. The analysis revealed that the relative increase of the subpopulation size is quite similar regardless of the densities with just few variations (see figure 3.8A, p.34). For MALP-2-stimulated microglia, significant differences between cell densities were primarily observed for the medium control and concentrations of 0.01 and $1 \mathrm{ng} / \mathrm{ml}$ while all the higher concentrations show no density-dependent differences. LPS-stimulated microglia show a similar increase, with significant differences for medium control, $0.1 \mathrm{ng} / \mathrm{ml}$ and $1 \mathrm{ng} / \mathrm{ml}$. $\mathrm{Pam}_{3} \mathrm{CSK}_{4}$-stimulated microglia showed a more heterogeneous increase and a rather sigmoidal growth curve. Significant differences between the densities were observed till a concentration of $10 \mathrm{ng} / \mathrm{ml}$.

Furthermore, I wanted to investigate the interplay between subpopulation size and the amount of secreted TNFa upon a cellular threat. I hypothesized that all secreting microglia secrete the same amount of TNFa and that microglia regardless of the density reach the same amount of total TNFa when facing the same threat. By assuming so, a higher microglia density should have a lower proportion of TNFa-secreting cells than lower microglia densities. However, analysis of the TNFa-secretion per cell revealed a density dependent secretion as well but in a reversed pattern (see figure 3.8B, p.34). The results showed that TNFa secretion per cell decreased with 
A

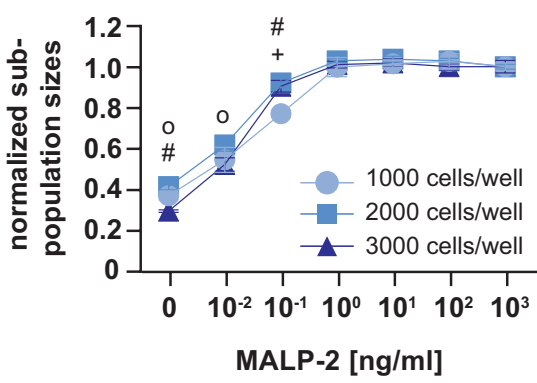

subpopulation size increase

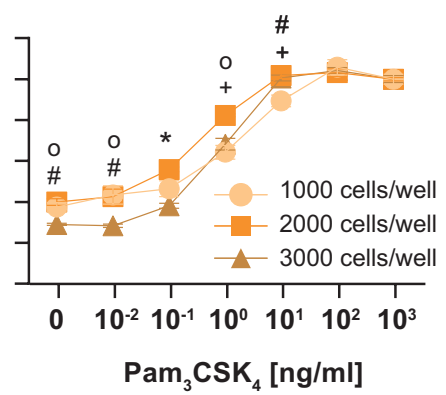

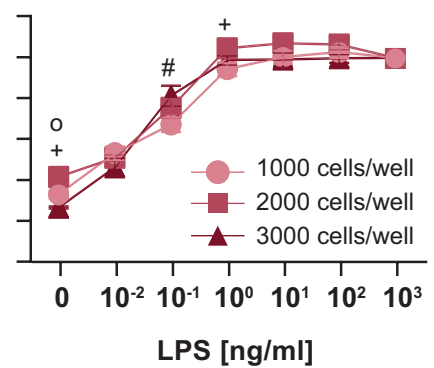

B

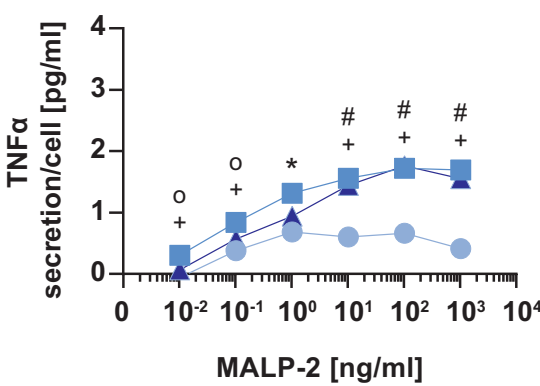

density dependent TNFa secretion per cell
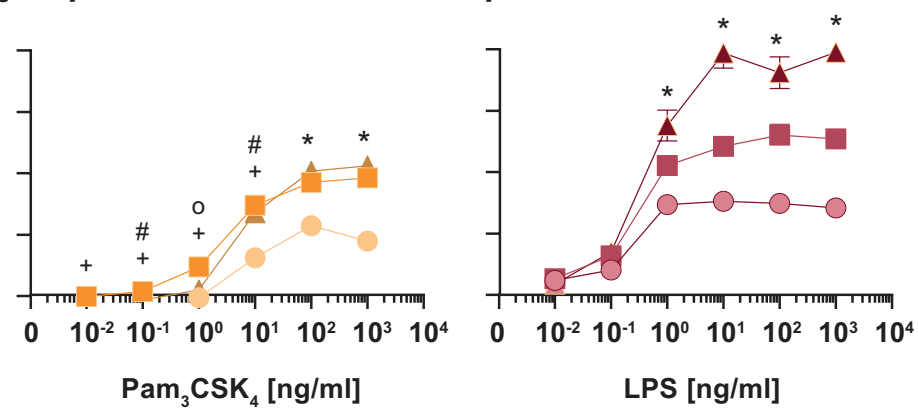

Figure 3.8 - Density dependent secretion per cell and subpopulation increase: A) Increase of the subpopulation sizes normalized to the highest value obtained for each stimuli and density. B) Denstiy dependent TNFa secretion per cell. Significant differences obtained via ANOVA and Tukey's multiple comparison with P-values $\leq 0.05$ were indicated as follows: * difference between all densities; + differences between 1000 and 2000 cells/well; \# differences between 1000 and 3000 cells/well; o differences between 2000 and 3000 cells/well. Data are presented as mean \pm SEM. $n=8$

decreasing microglia denstity. This was demonstrated in LPS-stimulated microglia at concentrations above $0.1 \mathrm{ng} / \mathrm{ml}$ between all densities. For MALP-2 and $\mathrm{Pam}_{3} \mathrm{CSK}_{4}$ stimulations differences were mostly significant between at least two of the densities with a similar increase in secretion for microglia plated at 2000 and 3000 cells/well and a lower one for 1000 cells/well.

Alltogether, it was shown that TNFa and RANTES-producing microglia subpopulation sizes depent on the microglia density, as the secreting subpopulation sizes decreased with increasing microglia denstity. The generell increase of the subpopulation sizes was barely affected by the density. Additionally, it was shown that microglia of higher densities produce less TNFa per cell than microglia of low densities. A corruption of the obtained data by increased cell death in higher densities could be excluded. 


\subsection{Microglia subpopulations overlap in their functions}

After identification of the TNFa and RANTES producing subpopulations, I investigated their composition via FACS analysis. The results show that subpopulations positive for TNFa, RANTES and both are already existing in unstimulated cells, though in relatively small proportions (see figure 3.9A).

A unstimulated control



B

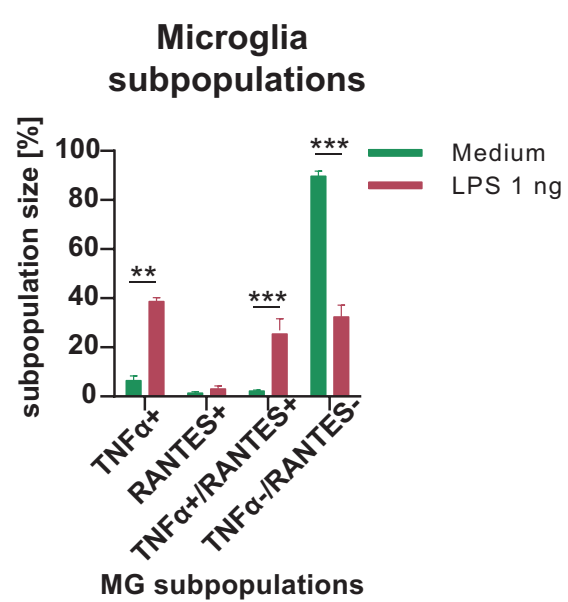

LPS stimulation

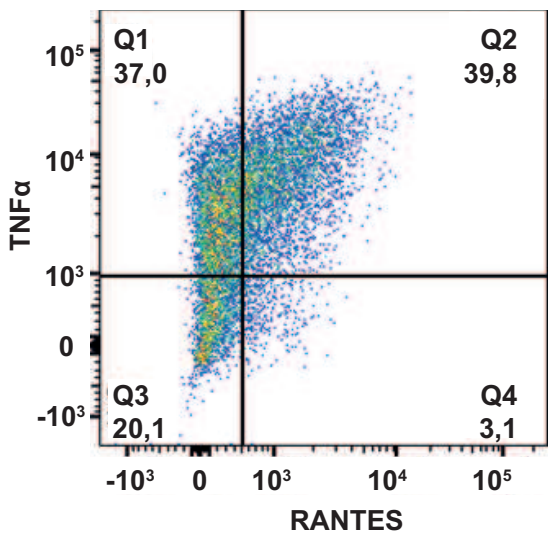

C

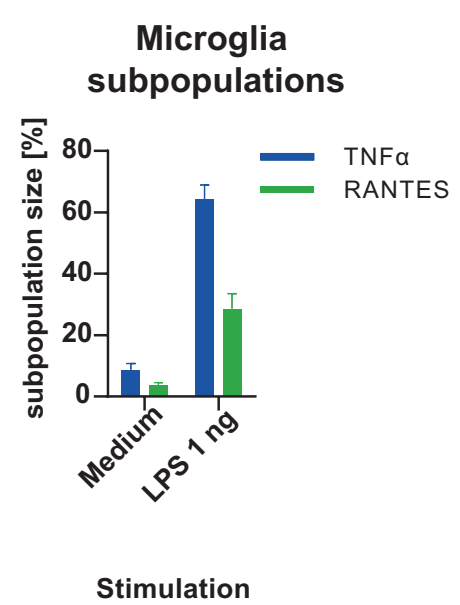

Figure 3.9 - Composition of TNFa- and RANTES-producing microglia subpopulation sizes analyzed via FACS: Microglia plated at 300000 cells/well were stimulated with medium or LPS $(1 \mathrm{ng} / \mathrm{ml})$ for 8 hours. TNFa- and RANTES-positive cells were analyzed via FACS. A) Representative FACS plots for unstimulated and stimulated microglia. B) Quantification of the individual subpopulations. C) Quantification of all TNFaand RANTES-positive cells. Data was analyzed via multiple t-test $\left({ }^{* *} \leq 0.005 ;{ }^{* * *} \leq 0.0005\right)$. Data are presented as mean \pm SEM. $n=6$ 
Once microglia are stimulated with $1 \mathrm{ng} / \mathrm{ml}$ LPS, the cytokine producing microglia population rises massively. Thereby, I could detect that TNFa-positive microglia are assembled by a population that is just positive for TNFa, which increases upon stimulation significantly from $6.4 \%$ to $38.8 \%$ and a subpopulation that is able to produce TNFa as well as RANTES, which is also increasing significantly upon stimulation from $2.3 \%$ to $25.5 \%$ (see figure $3.9 \mathrm{~B}, \mathrm{p} .35$ ). The RANTESpositive microglia population is also composed of a single and a double producing subpopulation of microglia. However, for RANTES the single positive microglia population stays more or less stable upon stimulation (increases insignificantly from $1.5 \%$ to $3.2 \%$ ) while the subopopulation which is able to produce both cytokines increases. The total amount of TNFa- and RANTESproducing microglia rises after stimulation from about $8.7 \%$ to $64.3 \%$ and $3.7 \%$ to nearly 28.7 $\%$, respectively (see figure 3.9C, p.35).

Thus, I could demonstrate that cytokine producing microglia are composed of microglia that secrete the investigated cytokine only and those that secrete multiple cytokines at once. The increase in cytokine release upon LPS stimulation is thereby heterogeneously regulated, as upregulation of RANTES is only conveyed by the subpopulation which secreted multiple cytokines while for TNFa the TNFa specific subpopulation increased as well. 


\subsection{Microglia proliferation and cytokine secretion}

As I could demonstrate that microglia are able to secrete multiple cytokines as once, I investigated how microglia subpopulations are composed in case of diverse functions, i.e. TNFa secretion and proliferation.
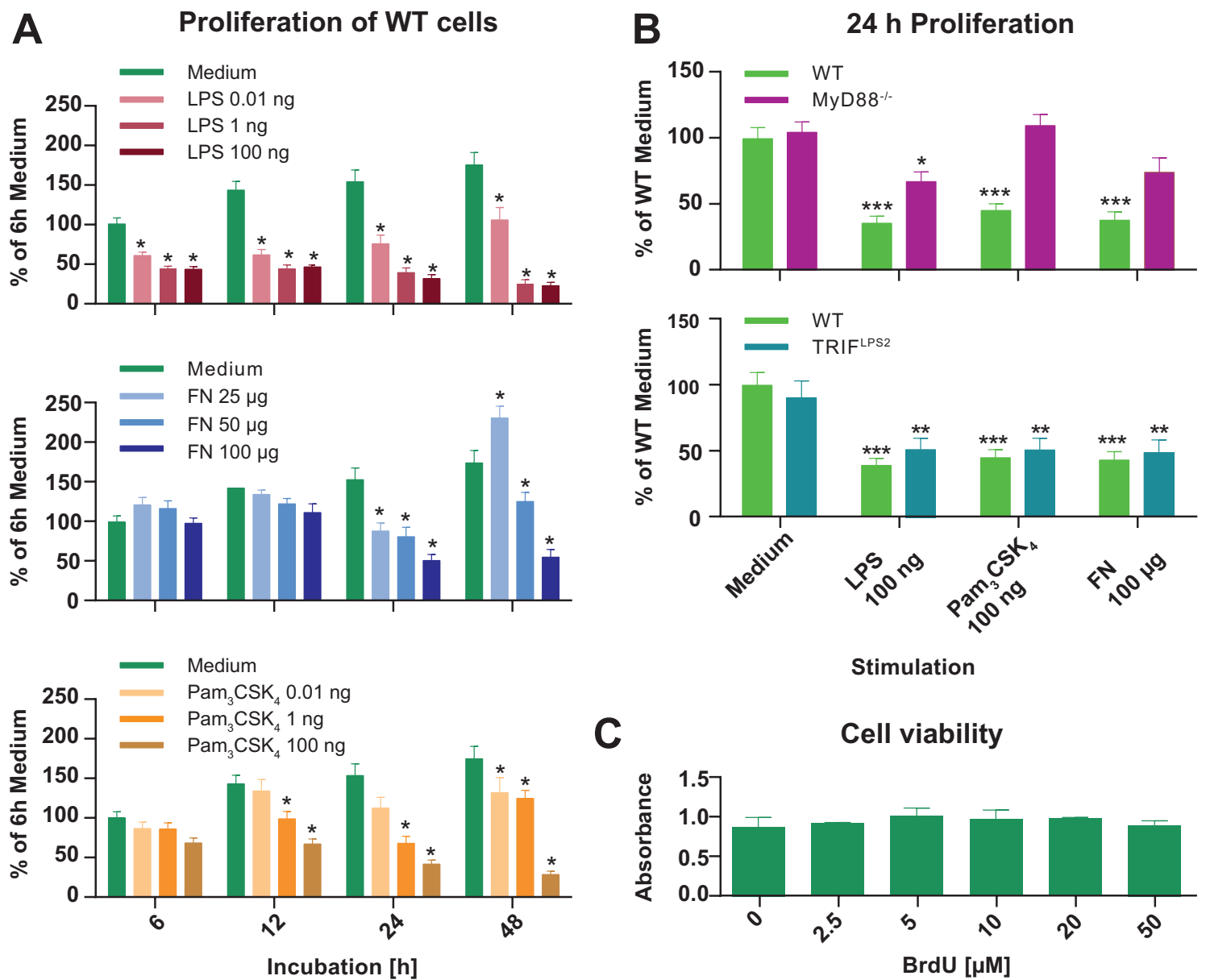

Figure 3.10 - Proliferation rate of challenged microglia isolated from WT and transgenic mice. A) Proliferation rate of WT microglia stimulated with $0.01 \mathrm{ng} / \mathrm{ml}, 1 \mathrm{ng} / \mathrm{ml}$ or $100 \mathrm{ng} / \mathrm{ml}$ of LPS or Pam ${ }_{3} \mathrm{CSK}_{4}$ or $25 \mu \mathrm{g} / \mathrm{ml}$, $50 \mu \mathrm{g} / \mathrm{ml}$ and $100 \mu \mathrm{g} / \mathrm{ml}$ of Fibronectin (FN); n= 14-20. B) Proliferation rate of myd88 ${ }^{-/-}$and trif ${ }^{\text {lps } 2}$ cells stimulated with $100 \mathrm{ng} / \mathrm{ml}$ of LPS and $\mathrm{Pam}_{3} \mathrm{CSK}_{4}$ or $100 \mu \mathrm{g} / \mathrm{ml}$ of FN for 24 hours. $\mathrm{n}=30-48$.The difference to WT medium levels was analyzed via 2-way ANOVA and Tukey's multiple comparison with asterisks indicating p-values $* 0.05 ;^{* *} \leq 0.005 ;{ }^{* * *} \leq 0.0005$. C) Viability assessment via the WST-1 assay of Microglia treated with increasing concentrations of BrdU for 24 hours. $n=6$. Data was analyzed via multiple t-test. Data are presented as mean \pm SEM. 
Since previous studies reported very controversial results on microglia proliferation upon challenges such as LPS stimulation (Gebicke-Haerter et al., 1989; Chen et al., 2012; Fukushima et al., 2015; George et al., 2015), I first examined the effect of any challenge on microglia proliferation, before investigating the temporal orchestration of proliferation and cytokine secretion. Therefore, I stimulated microglia with increasing concentrations of LPS, $\mathrm{Pam}_{3} \mathrm{CSK}_{4}$ (both $0.01 \mathrm{ng} / \mathrm{ml} ; 1$ ng/ml; $100 \mathrm{ng} / \mathrm{ml}$ ), and Fibronectin (FN) (25 $\mu \mathrm{g} ; 50 \mu \mathrm{g} ; 100 \mu \mathrm{g}$ ) for up to 48 hours and performed subsequently an ELISA based BrdU cell proliferation assay.

Analysis revealed that challenged microglia proliferate significantly less compared to untreated controls, and that this reduction becomes more pronounced the longer the challenge lasts (see figure 3.10A, p.37). Microglia stimulated with LPS showed a significant reduction in the proliferation rate for all tested incubation times and concentrations, ranging to a minimum of $22.1 \%$ compared to medium control. The FN-stimulated microglia showed a first impairment of proliferation after 24 hours for all used concentrations. At 48 hours, the decreasing effect seems to be reversed for low levels of FN ( $25 \mu \mathrm{g} / \mathrm{ml})$ but stayed consistent for the other concentrations. Here, the minimum proliferation rate reached $51.6 \%$ of the medium control. Microglia stimulated with $\mathrm{Pam}_{3} \mathrm{CSK}_{4}$ showed first reductions in the proliferation rate after 12 hours of incubation with $1 \mathrm{ng} / \mathrm{ml}$ and $100 \mathrm{ng} / \mathrm{ml}$. After 24 hours, the proliferation rate was significantly reduced for all used concentrations with a minimum of $28.6 \%$.

After demonstrating this reduction in the proliferation rate, I wanted to identify the responsible signaling pathway. Thus, I performed additional proliferation assays in myd88-/- and trif ${ }^{l p s 2}$ KO cells. For this purpose, I stimulated WT and KO microglia with high concentrations of the previously used stimuli for 24 hours (see figure 3.10B, p.37). A comparison between WT and

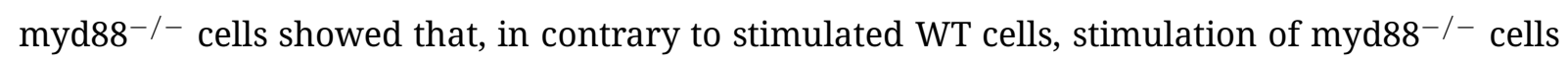
with $\mathrm{Pam}_{3} \mathrm{CSK}_{4}$ and Fibronectin did not reduce the proliferation rate significantly as it was observed in stimulated WT cells. Only LPS-stimulated KO cells showed a significant reduction compared to unstimulated WT cells. This reduction, however, was much weaker than in LPS-treated WT cells. In contrast to MyD88-/- cells, trif ${ }^{l p s 2}$ cells behaved similar to WT cells. The significant reduction after stimulation was demonstrated for all used stimuli. 
Additionally, I performed an WST-1 viability experiment with increasing concentrations of BrdU to exclude toxic effects of BrdU, which could bias the obtained proliferation data (see figure 3.10C, p.37). Microglia incubated with increasing concentrations of BrdU for 24 hours showed no differences in their viability compared to untreated control cells.

After assessment of the general proliferative behavior of challenged microglia, I tested if microglia proliferation and TNFa secretion occur simultaneously in the same cell once they are stimulated.

A unstimulated control

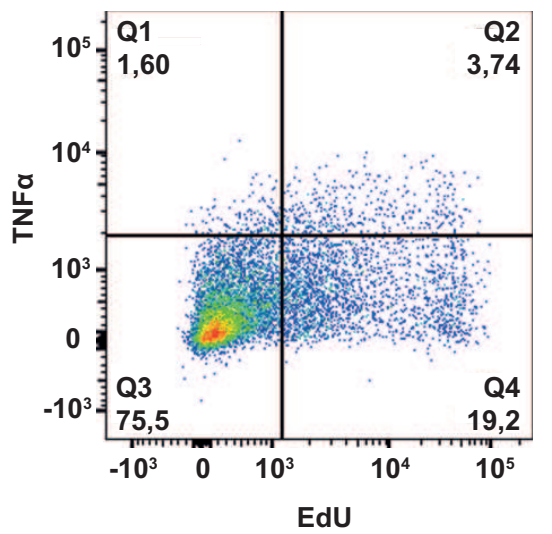

B

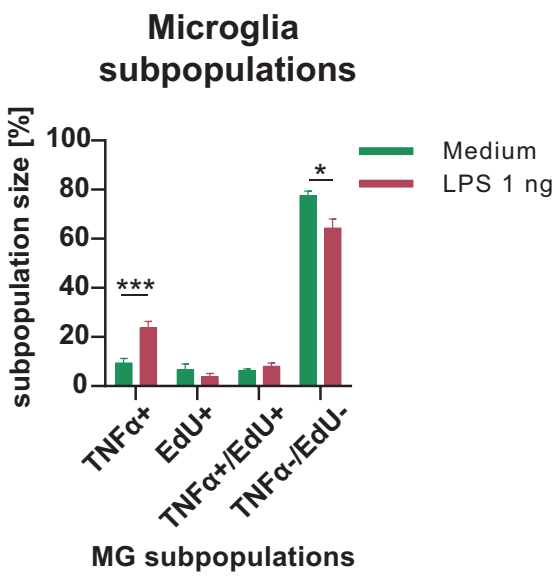

LPS stimulation

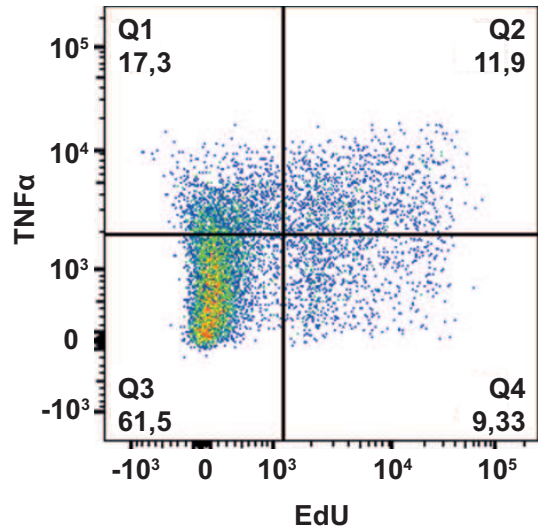

C

Microglia subpopulations

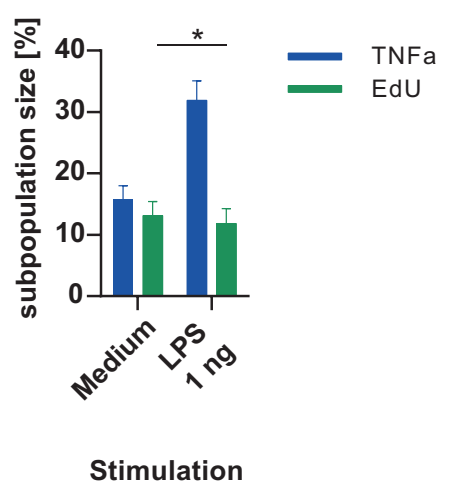

Figure 3.11 - Determination of TNFa-producing and proliferating microglia subpopulation sizes via FACS analysis: Microglia plated at 300000 cells/well were stimulated with medium or LPS ( $1 \mathrm{ng} / \mathrm{ml}$ ) containing 5 $\mu \mathrm{M}$ EdU for 8 hours. TNFa and EdU positive cells were analyzed via FACS. A) Representative FACS plots for unstimulated and stimulated microglia. B) Quantification of the individual subpopulations. C) Quantification of all TNFa and EdU positive cells. Data was analyzed via multiple t-test $\left(* \leq 0.05 ;{ }^{* * *} \leq 0.0005\right)$. Data are presented as mean \pm SEM. $n=7-8$ 
Hence, I performed combined FACS stainings for TNFa and EdU, a marker for proliferating cells. To do so, I stimulated microglia with $1 \mathrm{ng} / \mathrm{ml}$ LPS mixed with $5 \mu \mathrm{M}$ EdU for 8 hours. The obtained results of this double staining behaved quite similar to the previously performed one for TNFa and RANTES (see figure 3.11A, p.39). Unstimulated, TNFa-positive microglia are composed of a small population of TNFa secreting microglia (10.4\%) and a small population that is positive for both, secretion and proliferation (6.4 \%) (see figure 3.11B, p.39). This composition was also observed for the proliferating microglia subpopulation which included a subpopulation of dividing microglia with a size of $6.8 \%$.

LPS stimulation increased the TNFa producing subpopulation to $23.95 \%$, whereas the double positive microglia population was stable at a size of $8 \%$. The proliferating microglia subpopulation was not significantly affected by LPS stimulation but was slightly reduced to $3.8 \%$. However, the total percentage of proliferating microglia decreased significantly upon LPS stimulation from approximately $13.1 \%$ to 11.8 \% (see figure 3.11C, p.39), which con-

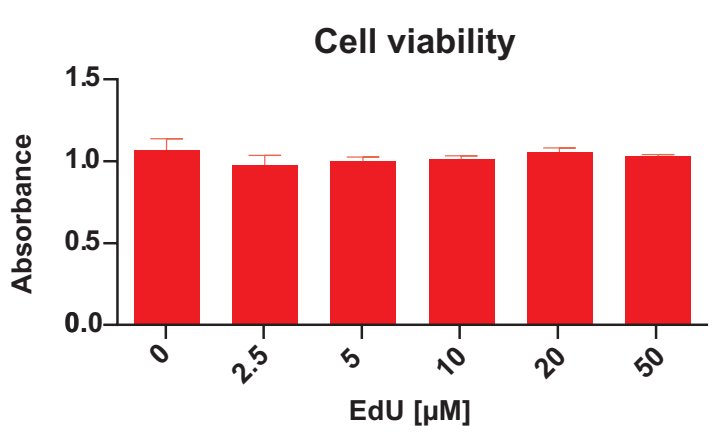

Figure 3.12 - Effect of EdU on microglia viability: microglia were treated with increasing concentrations of EdU for 24 hours. Viability was assessed via the WST-1 assay.Data was analyzed via multiple t-test. Data are presented as mean \pm SEM. $n=6$ firms my data obtained from the BrdU proliferation assay. The viability of microglia remained unchanged after a stimulation with increasing concentrations of EdU (see figure 3.12).

In summary, the proliferation rates in LPS, $\mathrm{Pam}_{3} \mathrm{CSK}_{4}$ and FN challenged microglia were reduced, primariliy driven by the Myd88 signaling pathway. In addition, microglia can multitask as some cells are able to perform proliferation and cytokine secretion simultaneously. A corruption of these results by a toxic effect of the used cell division markers could be excluded. 


\subsection{Effect of environmental cues on microglia subpopulation sizes}

Knowing that microglia subpopulation sizes are density dependent, the underlying mechanism conveying this effect is yet to be discovered. As microglia have mostly non-overlapping territories (Parkhurst and Gan, 2010) which excludes a contact-dependent organization, it is more likely that the secretion of certain substances could mediate the subpopulation sizes instead. As more microglia could produce higher amounts of a certain substance this increase in concentration could adjust the subpopuation sizes.

To test this hypothesis, I performed an insert experiment by plating 200000 microglia with or without transmembrane inserts containing additional 100000, 200000 or 300000 microglia cells. Stimulation with LPS results thus in supernatants produced by $200000-500000$ cells. Investigation of the plated 200000 cells via FACS showed that the TNFa-positive microglia proportion was not affected by the supernatants of increasing microglia densities (see figure 3.13). Since an absent effect could also be caused by an insufficient mixing of the supernatants, the cytokine production in- and outside of the inserts was compared as well. Significant differences for the tested factors were found for RANTES only. Here, supernatants from inside the inserts with 100000 and 200000 cells contained lower RANTES protein compared to supernatants from outside (see figure 3.14, p.42).

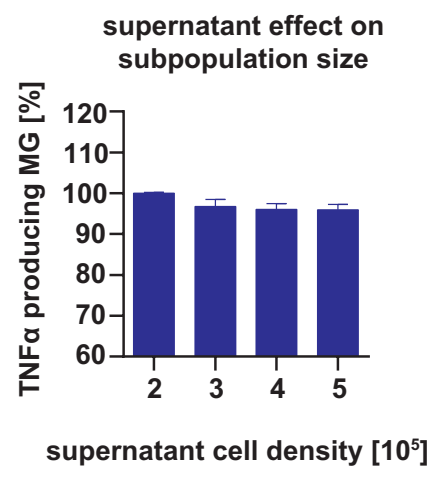

Figure 3.13 - Determination of supernatant effect on TNFa- producing microglia subpopulation size via FACS analysis: 200000 Microglia/well were plated with and without the presence of an insert containing additional 100000, 200000 or 300000 cells/insert and stimulated with $1 \mathrm{ng} / \mathrm{ml}$ LPS for 8 hours. The plated 200000 cells were subsequently analyzed for TNFa. FACS analysis of TNFa-producing microglia subpopulation size was normalized to values obtained from 200000 cells without inserts. Data was analyzed via multiple t-test. Data are presented as mean \pm SEM. $\mathrm{n}=8$ 
IL6

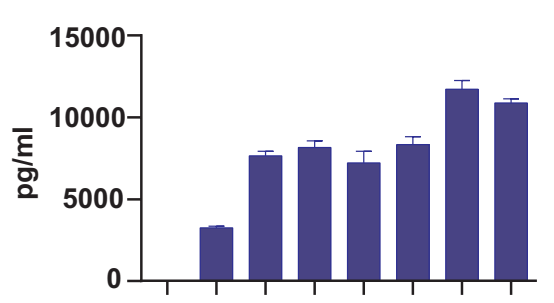

MIP1a

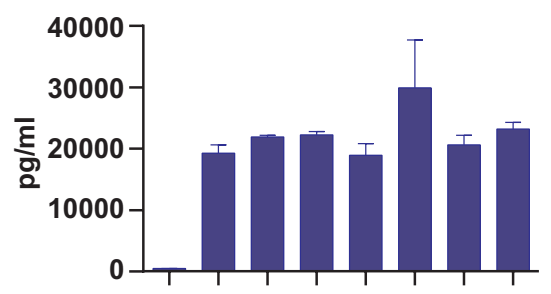

TNFa

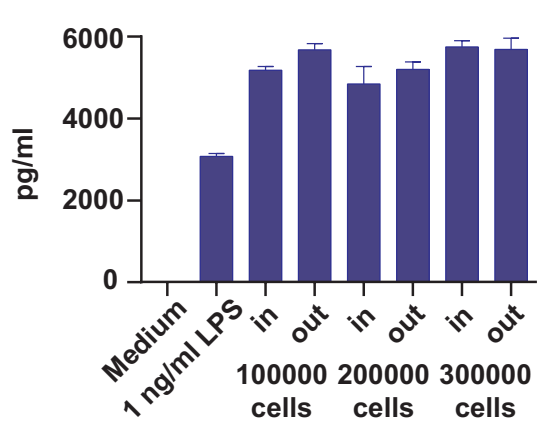

IL12-p40

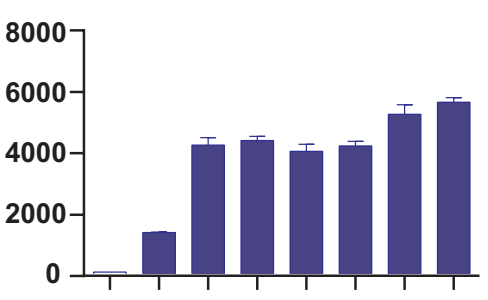

MCP1

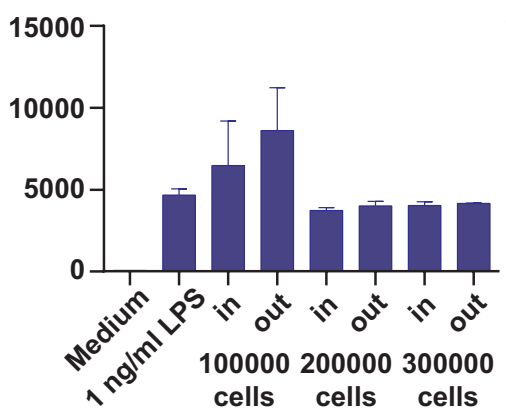

KC

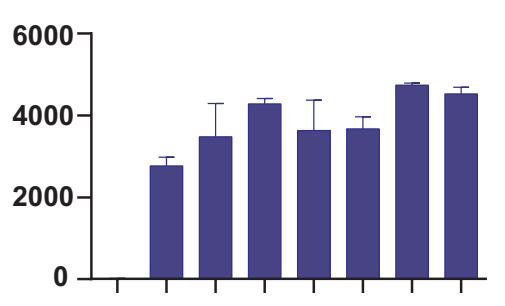

RANTES

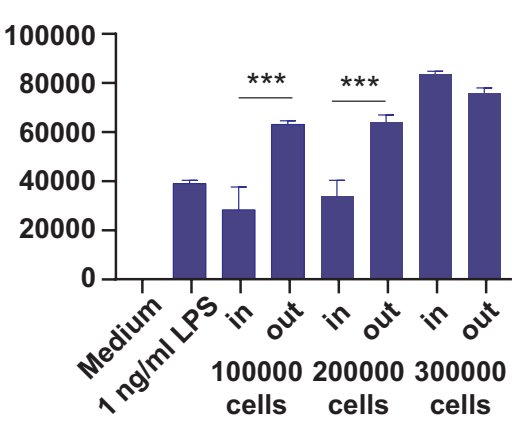

Figure 3.14 - Cytokine levels inside and outside of inserts: Supernatant from inside and outside the insert of the insert experiment were analysed via ELISA to determine the cytokine secretion of IL6, IL12-p40, KC, MIP1a, MCP-1, RANTES and TNFa. Data was analyzed via ANOVA and Tukey's multiple comparison with asterisks indicating $\mathrm{p}$-values ${ }^{* * *} \leq 0.0005$. Data are presented as mean \pm SEM. $\mathrm{n}=4$

To avoid the limitations caused by the insert experiment, an supernatant transfer experiment with supernatants from different densities with different treatments was performed. The first experiment revealed no differences in the TNFa-producing subpopulation size between the conditions but a significant difference between the LPS-treated groups (see figure 3.15A, p.43). The subpopulation sizes were significantly smaller in the groups that received supernatant from the cells that were already stimulated for 8 hours with LPS compared to the groups that received LPS after the supernatant transfer. A comparison of the supernatants from 200000 and 500000 cells for each group shows no effect on the subpopulation size. 
To exclude that this decline is caused by a possible exhaustion of the LPS amount in the prestimulated condition, the experiment was repeated with a higher LPS concentration $(10 \mathrm{ng} / \mathrm{ml})$ and subpopulation sizes of TNFa-and RANTES-producing microglia were investigated. These repetitions were performed together with the master student Robin Hindmarsh. Again, the subpopulation size regarding TNFa did not change between the 200000 cells and the 200000 cells that received supernatant from 500000 cells, independent of the stimulation paradigm (see figure 3.15B), which also holds true for RANTES (see figure 3.15C). The TNFa-producing subpopulation sizes of microglia that were stimulated with LPS after the supernatant transfer (approximately $77 \%$ differed significantly from the sizes of microglia supplemented with already stimulated supernatant (approximately $55 \%$ ). This effect of the pre-stimulation could not be observed for RANTES.

\section{A TNFa-producing subpopulation size}

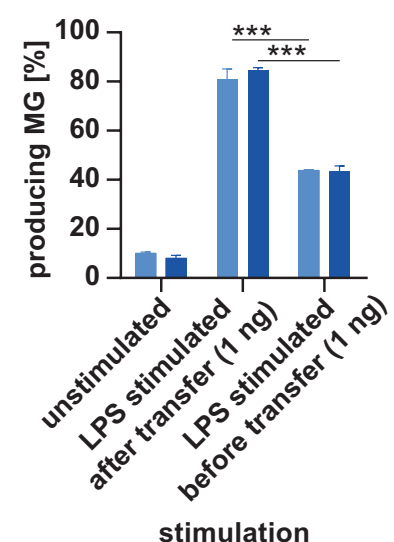

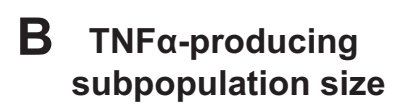

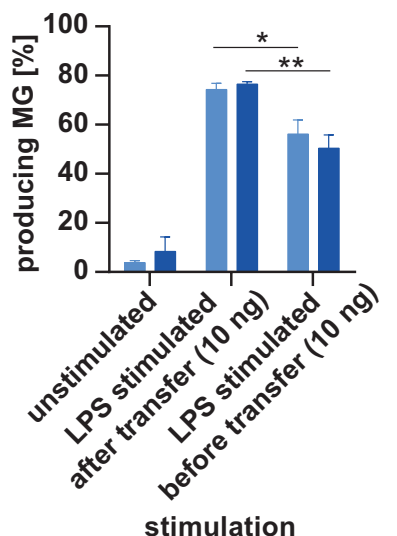

\section{RANTES-producing subpopulation size}

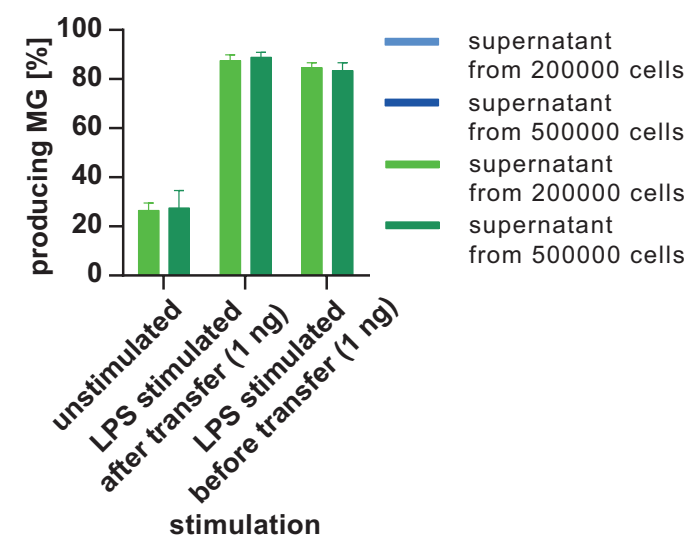

Figure 3.15 - Supernatant transfer experiment: 200000 or 5000000 microglia cells plated in 12-well plates were treated as the following: 200000 cells and 200000 cells that received the supernatant of 500000 unstimulated cells for 8 hours served as unstimulated controls. 200000 cells and 200000 cells with the supernatant of 500000 unstimulated cells were stimulated with LPS for 8 hours. 200000 cells were supplemented for 8 hours with the supernatants of 200000 or 500000 cells that were already stimulated with LPS for 8 hours beforehand. A) Microglia were stimulated with $1 \mathrm{ng} / \mathrm{ml} \mathrm{LPS} \mathrm{and} \mathrm{analyzed} \mathrm{for} \mathrm{the} \mathrm{TNFa-producing}$ subpopulation size. $\mathrm{n}=2$. B) Microglia were stimulated with $10 \mathrm{ng} / \mathrm{ml}$ LPS and analyzed for the TNFaproducing subpopulation and C) the RANTES-producing subpopulation size. $\mathrm{n}=8$. Data was analyzed via ANOVA and Tukey's multiple comparison with asterisks indicating significant differences between LPS stimulated groups with p-values $* \leq 0.05{ }^{* *} \leq 0.005 ; * * * \leq 0.0005$. Data are presented as mean \pm SEM. These experiments were partially performed together with the master student Robin Hindmarsh. 
In conclusion, it was demonstrated that the supernatant of LPS stimulated microglia can but doesn't have to affect the microglia subpopulation sizes, while supernatants of different densities seems to have no effect. 


\subsection{TNFa effect on TNFa secretion}

As the secreted cytokines themselves could affect their own secretion or secretion of other cytokines in a feedback loop, leading to a reduction or increase in production over time I examined this possible effect as well. To test this, ELISA experiments of microglia pre-stimulated with TNFa for different durations, ranging from 5 minutes to 24 hours with a subsequent LPS stimulation were perfomed. Pre-stimulated microglia that received medium instead of LPS served as controls. The analysis of the amounts of secreted cytokines revealed that TNFa pre-incubation alone was not able to induce significant cytokine secretion. However, with a successive LPS stimulation, TNFa affected the production of most measured cytokines after a pre-incubation period of more than 6 hours (see figure 3.16, p.46). The cytokines RANTES, IL12-p40, TNFa and IL6 are significantly reduced after a pre-incubation period of 6 hours or longer. A significant reduction for KC emerges at 12 hours. MCP-1 was the only measured cytokine which was unaffected by the TNFa pre-stimulation. Only a single cytokine showed a significant increase in release after incubation periods of 6 hours and higher, namely MIP1a. After observing this effect of TNFa preincubation on cytokine secretion, the experiment was repeated with pre-incubations of RANTES, MCP-1 and MIP1a. These repetitions were carried out together with the bachelor student Timo Pauw.

As seen in figure 3.17, pre-incubation with RANTES showed no effect on the cytokine secretion. While the pre-incubation alone was not able to induce cytokine secretion, the combination with LPS also had no effect on the amount of secreted cytokines.

Pre-incubation with MCP-1 showed minor effects on all cytokines except RANTES (see figure 3.18, p.48). A pre-incubation with MCP-1 alone induced no cytokine production, but the combination with LPS stimulation induced various changes. IL6, IL12-p40 and KC showed a significant reduction in their secretion after 24 hours of pre-incubation time. For KC an additional increase was observed after 3 hours which was absent again at longer incubation periods. TNFa and MIP1a showed significant increases after $3 \mathrm{~h}$ and $6 \mathrm{~h}$, respectively. MCP-1 levels showed differences at 10 minutes, 25 minutes and 6 hours and RANTES secretion was not affected by any pre-incubation period. 
IL6

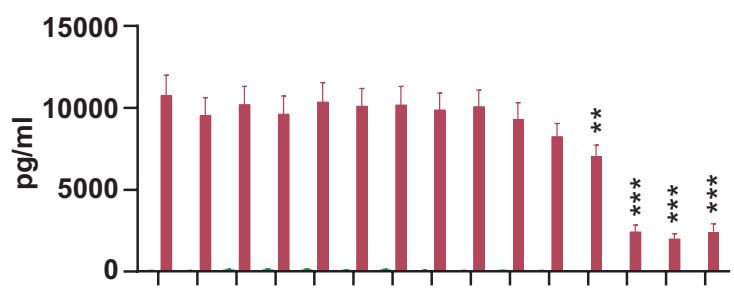

KC

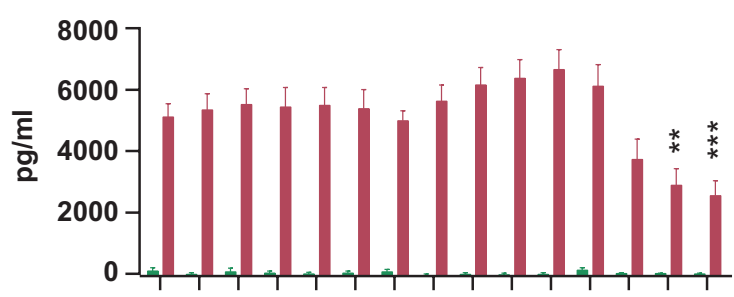

TNFa

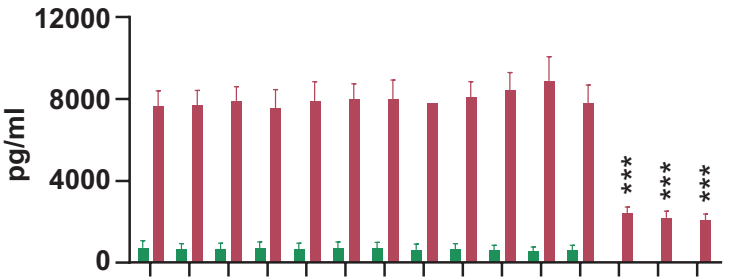

MCP-1

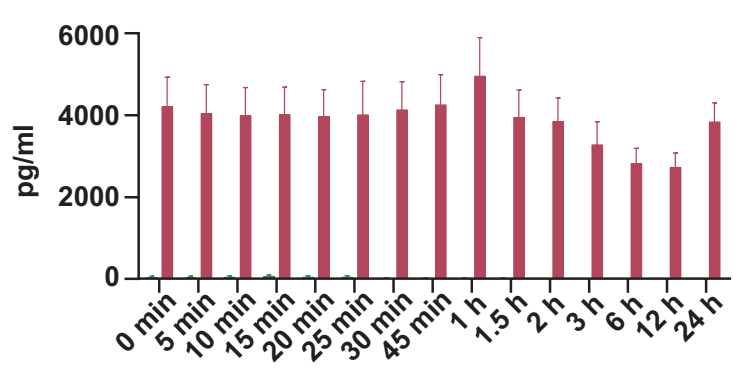

pre-incubation time
IL12-p40



RANTES

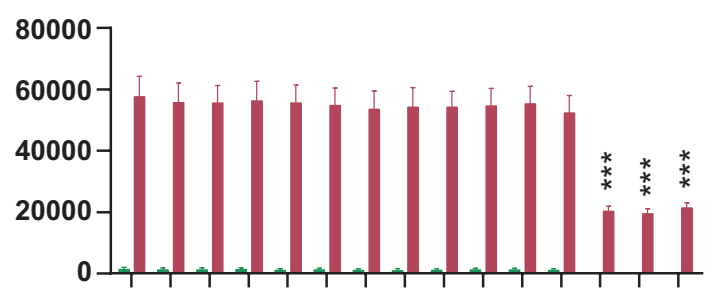

MIP1a

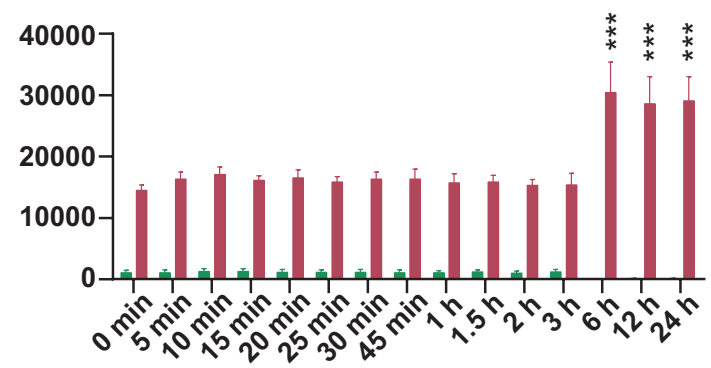

pre-incubation time

Medium

Figure 3.16 - Time dependent effect of TNFa pre-incubation on microglial cytokine and chemokine production in general and upon LPS stimulation: Microglia were pre-incubated with $10 \mathrm{ng} / \mathrm{ml}$ TNFa for the indicated time points. After removing TNFa cells were subsequently incubated with fresh medium or stimulated with LPS ( $1 \mathrm{ng} / \mathrm{ml})$ for 18 hours. Chemokine and Cytokine production was assessed using ELISA. Data was analyzed via Two Way ANOVA and Tukey's multiple comparison with asterisks indicating significant differences between LPS stimulated groups with p-values ${ }^{* *} \leq 0.005 ;{ }^{* *} \leq 0.0005$. Data are presented as mean \pm SEM. $\mathrm{n}=16$ 
IL6
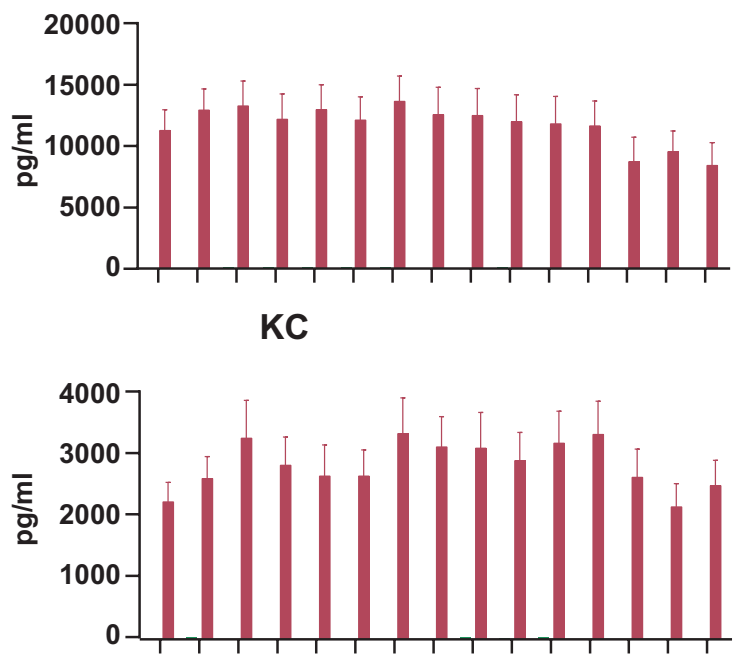

TNFa

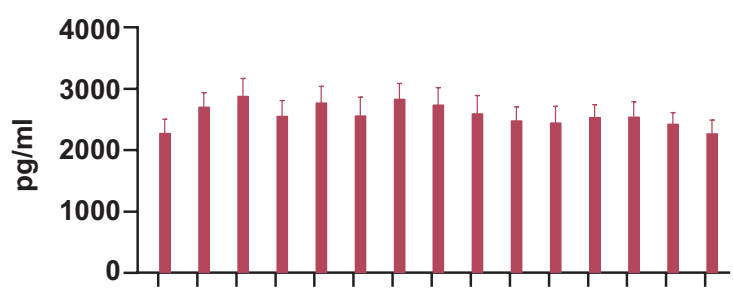

MCP-1



IL12-p40



RANTES

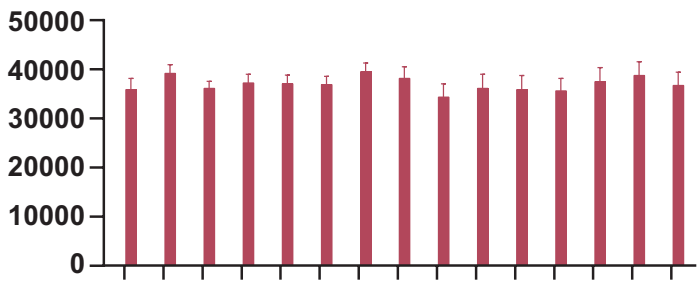

MIP1a

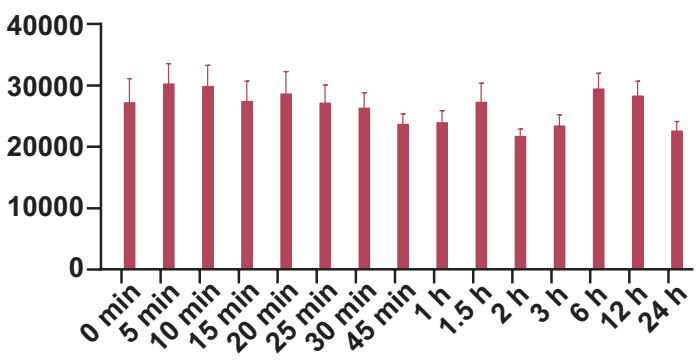

Figure 3.17 - Time dependent effect of RANTES pre-incubation on microglial cytokine and chemokine production in general and upon LPS stimulation: Microglia were pre-incubated with $0.1 \mathrm{ng} / \mathrm{ml}$ RANTES for the indicated time points. After removing RANTES cells were subsequently incubated with fresh medium or stimulated with LPS ( $1 \mathrm{ng} / \mathrm{ml})$ for 18 hours. Chemokine and Cytokine production was assessed using ELISA. Data was analyzed via Two Way ANOVA. Data are presented as mean \pm SEM. $n=16$. This experiment was carried out together with the bachelor student Timo Pauw. 
IL6

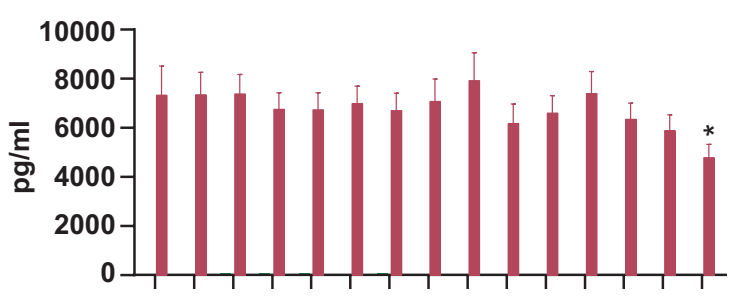

KC

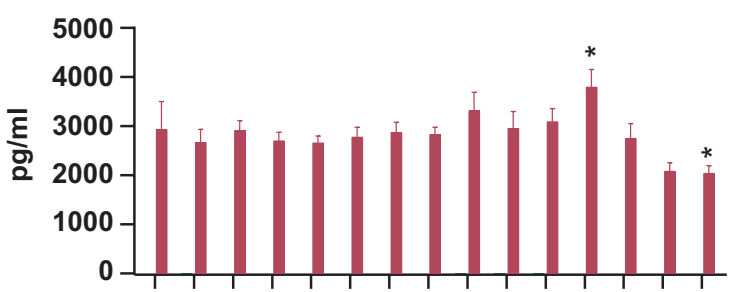

TNF $\alpha$

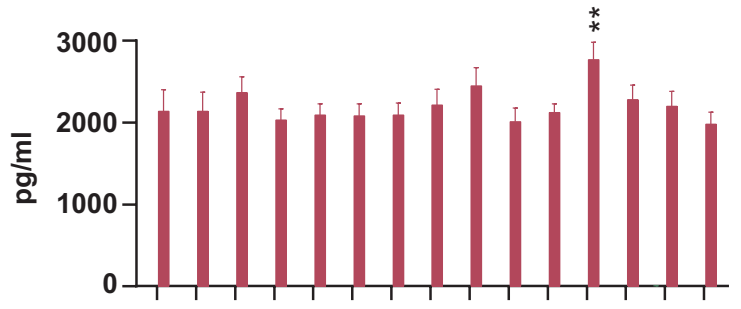

MCP-1

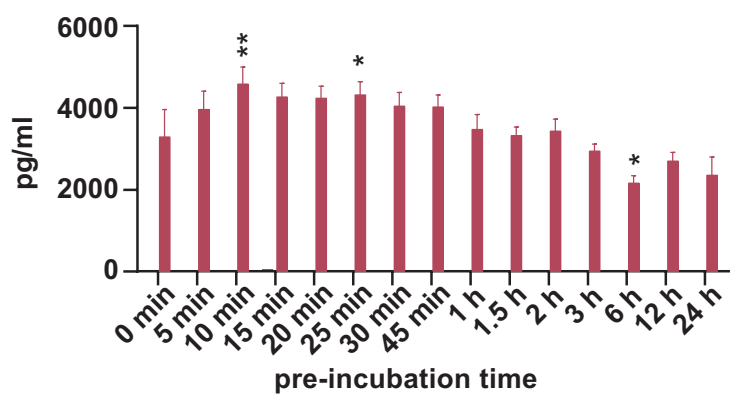

IL12-p40

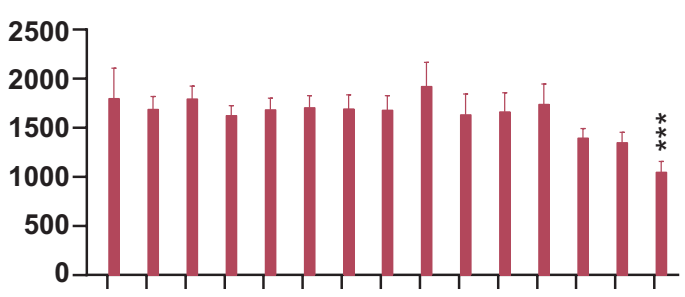

RANTES

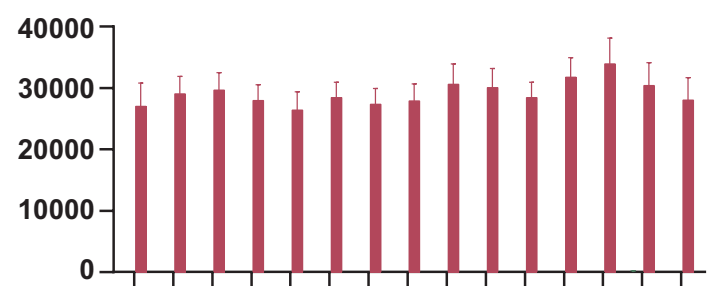

MIP1a

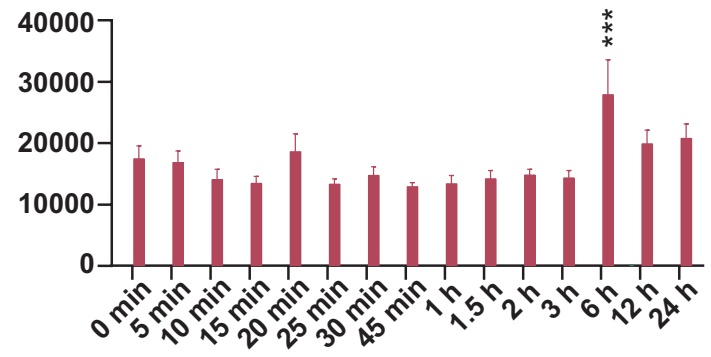

pre-incubation time

Medium LPS $1 \mathrm{ng}$

Figure 3.18 - Time dependent effect of MCP-1 pre-incubation on microglial cytokine and chemokine production in general and upon LPS stimulation: Microglia were pre-incubated with $0.1 \mathrm{ng} / \mathrm{ml} \mathrm{MCP-1}$ for the indicated time points. After removing MCP-1 cells were subsequently incubated with fresh medium or stimulated with LPS (1 ng/ml) for 18 hours. Chemokine and Cytokine production was assessed using ELISA. Data was analyzed via Two Way ANOVA and Tukey's multiple comparison with asterisks indicating significant differences between LPS stimulated groups with $p$-values $* * \leq 0.005 ; * * * \leq 0.0005$. Data are presented as mean \pm SEM. $n=16$. This experiment was carried out together with the bachelor student Timo Pauw 
IL6

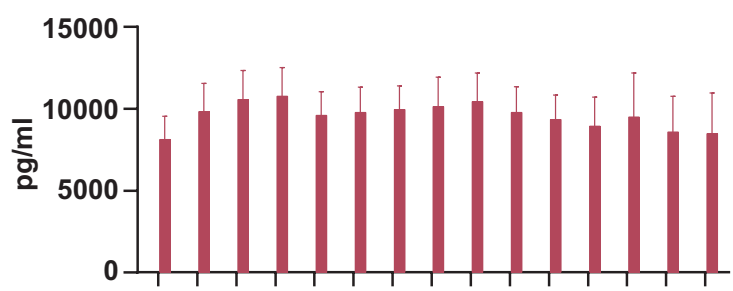

KC

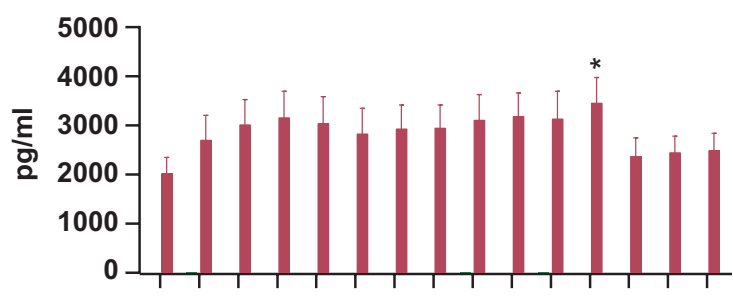

TNFa

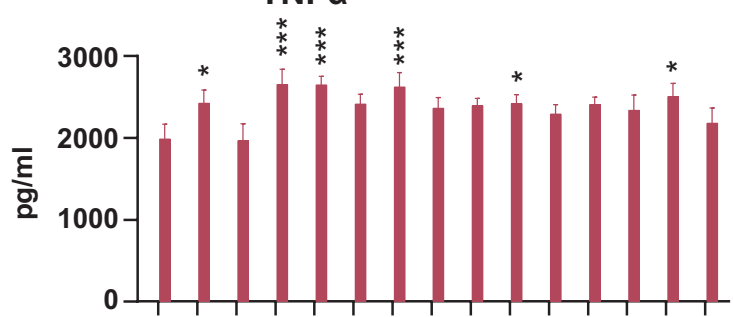

MCP-1

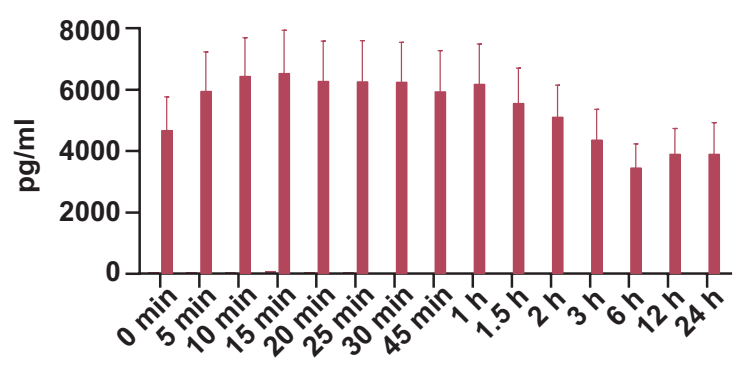

IL12-p40

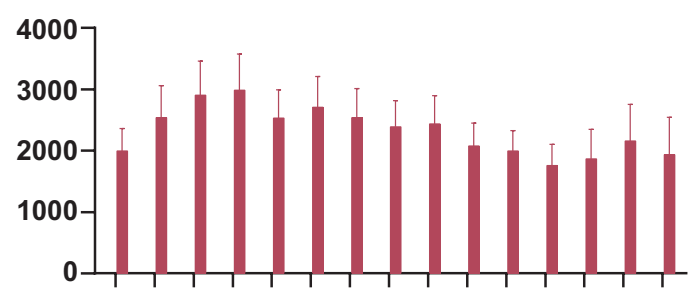

RANTES



MIP1a

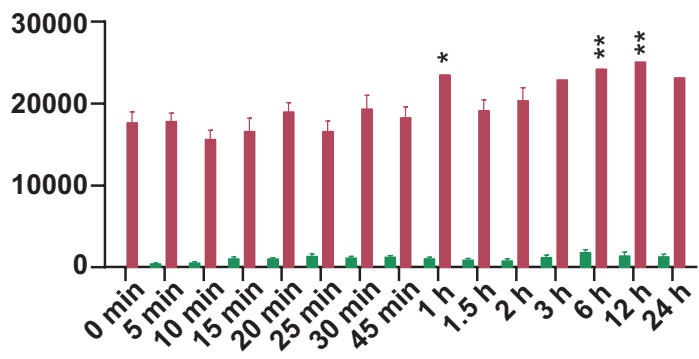

pre-incubation time

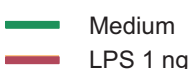

Figure 3.19 - Time dependent effect of MCP-1 pre-incubation on microglial cytokine and chemokine production in general and upon LPS stimulation: Microglia were pre-incubated with $100 \mathrm{ng} / \mathrm{ml} \mathrm{MIP1a}$ for the indicated time points. After removing MIP1a cells were subsequently incubated with fresh medium or stimulated with LPS (1 ng/ml) for 18 hours. Chemokine and Cytokine production was assessed using ELISA. Data was analyzed via Two Way ANOVA and Tukey's multiple comparison with asterisks indicating significant differences between LPS stimulated groups with p-values ${ }^{* *} \leq 0.005 ;{ }^{* * *} \leq 0.0005$. Data are presented as mean \pm SEM. $n=16$. together with the bachelor student Timo Pauw. 
A pre-incubation with MIP1a in combination with LPS stimulation affected the secretion of KC, RANTES, TNFa and MIP1a (see figure 3.19, p.49). While IL6, IL12-p40 and MCP-1 secretions were unaffected, the secretion of the other cytokines was elevated. KC levels were increased at 3 hours of pre-incubation time. RANTES levels increased significantly after 5 minutes and stayed relatively stable afterwards. TNFa showed a significant increase at 5, 15, 20 and 30 minutes as well as 1.5 and 12 hours of pre-incubation time. MIP1a pre-incubation enhanced its own secretion after $1 \mathrm{~h}, 6 \mathrm{~h}$ and 12 hours of pre-incubation time. Cells pre-incubated with MIP1a that received medium instead of LPS had no significant effect on the tested cytokines.

Since TNFa was the only tested cytokine which was able to induce a strong and consistent effect on the cytokine levels, I investigated the effect of TNFa in more detail. First, an LDH assay was perfomed to
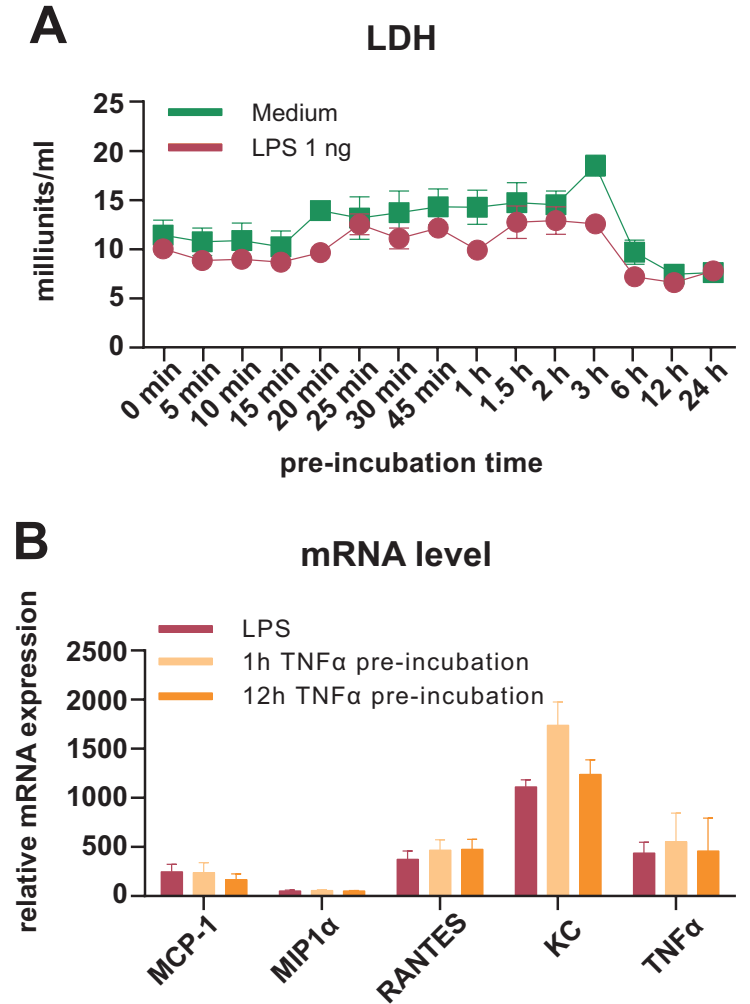

Figure 3.20 - Effect of TNFa on microglia viability and RNA levels of cytokines: A) Supernatants from the TNFa pre-incubation experiments were used to determine the viability of microglia via the LDH assay. B) RNA was extracted from microglia prestimulated with $10 \mathrm{ng} / \mathrm{ml}$ TNFa for $0 \mathrm{~h}, 1 \mathrm{~h}$ and $12 \mathrm{~h}$ and stimulated with $1 \mathrm{ng} / \mathrm{ml}$ LPS for 3 hours. mRNA levels were measured by RT-PCR, relative mRNA expression for MCP-1, MIP1a, RANTES, KC and TNFa was calculated by $2^{-\triangle \Delta C T}$ to medium controls with Gapdh as the control gene. Data was analyzed via ANOVA. Data are presented as mean \pm SEM. $n=4$

exclude a toxicity effect of TNFa on the microglial cells due to an excessinve incubation. Upon tissue damage or toxicity, cells release LDH which leads to an increase in its concentration. No significant increases between the 0-minute control and the other pre-incubation times for the LPS stimulated microglia could be detected (see figure 3.20A). Pre-stimulated microglia that received medium instead of LPS afterwards also showed no changes in their viability. 

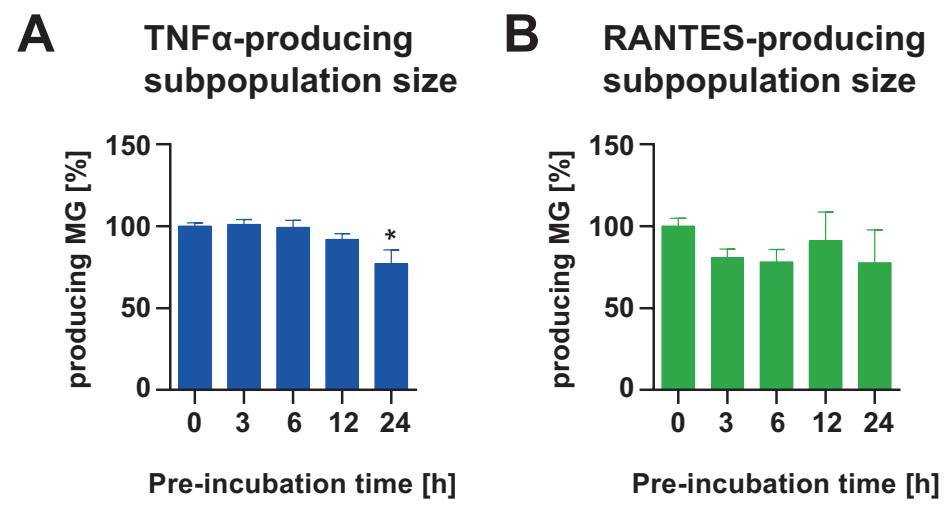

Figure 3.21 - FACS analysis of TNFa pre-incubation effect on TNFa- and RANTES-producing microglia subpopulation sizes: Microglia were pre-stimulated with $10 \mathrm{ng} / \mathrm{ml}$ TNFa for $0 \mathrm{~h}, 1 \mathrm{~h}, 3 \mathrm{~h}, 6 \mathrm{~h}, 12 \mathrm{~h}$ and stimulated with $1 \mathrm{ng} / \mathrm{ml}$ LPS for 8 hours. Data was normalized to values obtained from $0 \mathrm{~h}$ control cells and analyzed via ANOVA and Tukey's multiple comparison with asterisks indicating significant differences between LPS stimulated groups with $p$-values $* \leq 0.05$. Data are presented as mean \pm SEM. $n=8$

Next, I investigated if the observed TNFa effect on cytokine secretion is also detectable on the level of transcription using qPCR. Therefore, microglia were pre-incubated with $10 \mathrm{ng} / \mathrm{ml}$ TNFa for 0,1 and 12 hours and subsequently stimulated with LPS for 3 hours. The mRNA levels of the tested cytokines relative to the levels obtained from the negative control revealed no differences between the three treatment groups of all tested cytokines (see figure 3.20B, p.50). I also wanted to see if the observed effect of TNFa could affect the cytokine secretion by changing the size of the producing subpopulation. Thus, I repeated the pre-incubation experiment in a FACS approach for up to 24 hours of pre-incubation time and $8 \mathrm{~h}$ LPS stimulation. The data revealed a reduction in the TNFa-producing subpopulation size to $77.1 \%$ of the control after a pre-incubation period of 24 hours (see figure 3.21A). The RANTES-producing subpopulation size was not affected by the TNFa pre-incubation (see figure 3.21B). Since ELISA and FACS experiments were performed with different incubation times for LPS (18 and 8 hours, repectively), the observed insignificant change might be caused by an insufficient incubation time with LPS. Thus, a control ELISA experiment was performed with TNFa pre-stimulated microglia, stimulated with LPS for 8 hours. As it is shown in figure $3.22,8$ hours of LPS incubation is insufficient to elicit the same effect that was observed with 18 hours of LPS stimulation on TNFa and RANTES secretion (see figure 3.22, p.52). While IL6, IL12-p40 and MCP-1 showed a reduction in secretion after 12 hours of pre-incubation time, the levels of KC, MIP1a, RANTES and TNFa were not affected at any preincubation duration. 
IL6


IL12-p40

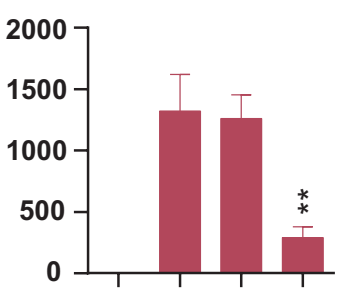

RANTES

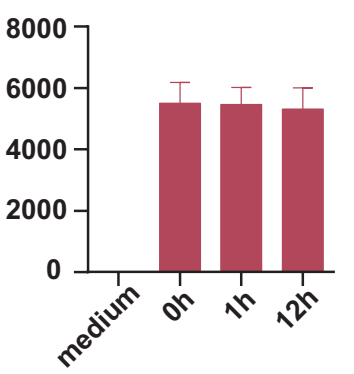

KC

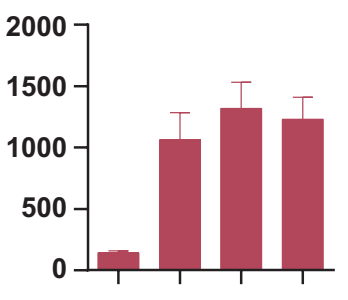

TNFa

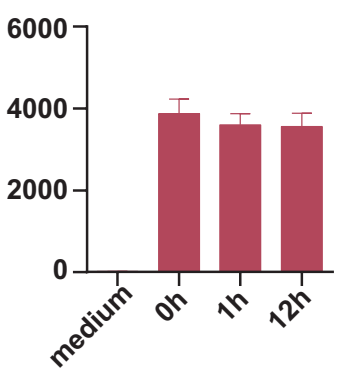

MCP-1



pre-incubation time

pre-incubation time

Figure 3.22 - Effect of TNFa pre-incubation on microglial cytokine and chemokine production after 8 hours of LPS stimulation: Microglia plated at a density of 15000 cells in a 96-well format were pre-incubated with $10 \mathrm{ng} / \mathrm{ml} \mathrm{TNFa}$ for $0 \mathrm{~h}, 1 \mathrm{~h}$ and 12 hours and subsequently stimulated with LPS ( $1 \mathrm{ng} / \mathrm{ml})$ for 8 hours. Microglia without pre-stimulation that received fresh medium instead of LPS served as medium controls. Chemokine and Cytokine production was assessed using ELISA. Data was analyzed via ANOVA and Tukey's multiple comparison with asterisks indicating $\mathrm{p}$-values ${ }^{* *} \leq 0.005 ;{ }^{* * *} \leq 0.0005$. Data are presented as mean \pm SEM. $\mathrm{n}=16$

Taken together, only a fraction of cytokines was able to regulate and self-adjust cytokine secretion levels. TNFa had the strongest effect of all tested cytokines even though this effect was absent on mRNA level. FACS analysis demonstrated an influence of TNFa pre-incubation on the TNFa-producing microglial subpopulations exclusively. A possible cause for the variations in the effect of the ELISA and FACS experiments could be the incubation time for LPS, as the effect of TNFa pre-incubation on some cytokines could be only observed after 18 hours but not after 8 hours of LPS stimulation. 


\section{Discussion}

Microglia are known to be implicated in neurodevelopmental, neuroinflammatory, neurodegenerative and neuropsychiatric diseases ((reviewed in Block and Hong, 2005). Immune dysregulation caused by the diseased environment leads to over-activation, exaggerated and/ or inhibited functions of microglia, which are in most cases associated with neuroinflammation, worsening the diseases (Maezawa and Jin, 2010; Suzuki K et al., 2013). Treatments aiming to control this neuroinflammation as therapeutic approaches to oppose the diseases are thus under extensive investigation. And indeed, antipsychotics, for instance ones used for schizophrenia, are known to decrease the secretion of several pro-inflammatory factors like TNFa or nitric oxide, suggesting a potentially useful therapeutic effect (Kato et al., 2008, 2007; Kowalski et al., 2003; Sugino et al., 2009). However, some antipsychotics increase the amount of other pro-inflammatory cytokines as well (Himmerich et al., 2011; Kluge et al., 2009). This phenomenon and the fact that microglia also display beneficial, immune-resolving functions, questions the usefulness of unspecific anti-inflammatory drugs (Biber et al., 2016). Thus, with the emerging awareness of microglia subpopulations, it would be of utmost importance to identify drugs which specifically target the aversive microglia while leaving the protective ones unaffected or even supported (Kamigaki et al., 2016; London et al., 2013). An understanding of microglia subpopulations and their organization would be the requirement to accomplish the identification of specific drugs. Though, research addressing this issue is scarcely existing so far. This study investigated the behavior and organization of different microglia subpopulations to shed some light on this so far unknown but highly important subject. 


\subsection{Microglia subpopulation sizes are dynamically adapted in response to different challenges}

Microglia are able to respond to specific stimuli in a dose dependent manner. This was shown for LPS, $\mathrm{Pam}_{3} \mathrm{CSK}_{4}$, staphylococcus aureus, escherichia coli, fibronectin and Lipid A conducted in in vivo and/or in vitro studies for a broad range of cytokines like TNFa (Janova et al., 2016; Kielian et al., 2002; Lund et al., 2006; Mildner et al., 2007; Regen et al., 2011). Even though, the underlying signaling mechanisms are known in most cases, the organizational principle of the change in the secreted amount was not investigated so far. The lack of investment can be explained by the former assumption that microglia are a homogenous population of immune cells (reviewed by Hanisch, 2013). This assumption would allow just one explanation for the change in the cytokine levels - the secretion per cell has to be altered. However, research from the recent decade strongly suggests a rather heterogeneous character of microglia, offering another hypothesis which might explain the change in cytokine quantities: Alterations in the relative portion of secreting cells (Baalman et al., 2015; Butovsky et al., 2006; Chen et al., 2010; Clausen et al., 2008; Fang et al., 2014; Färber et al., 2005; Gertig and Hanisch, 2014; Kawahara et al., 2009; Kobayashi et al., 2013; Kocur et al., 2015; Kuhn et al., 2004; Marshall et al., 2014; Noda et al., 2000; Pannell et al., 2014b; Pepe et al., 2014; Schmid et al., 2002; Shinjo et al., 2014; Trias et al., 2013; Venkatesan et al., 2010; Wlodarczyk et al., 2015). In the present study, I addressed this question of how the change in cytokine secretion is organized by investigating TNFa, a cytokine which was already demonstrated to be produced by a subpopulation of microglia only, and RANTES, a cytokine that was not investigated regarding subpopulations so far (Scheffel et al., 2012).

By testing the effect of increasing concentrations of LPS, Pam $_{3} \mathrm{CSK}_{4}$ and MALP-2 on TNFa- and RANTES-producing microglia I was able to demonstrate a variety of important features. Both TNFa- and RANTES were secreted by microglia subpopulations, which confirms the findings from Scheffel et al. and adds RANTES production to the list of subpopulational features. As both randomly chosen cytokines characterize subpopulations of microglia, this characterization might be a general feature of all cytokines, as it was shown for Interleukin 1 beta as well (Clausen et al., 2008). Thus, at the first glance, the development of drugs specifically targeting cytokine producing subpopulations would be feasible. 
In addition, I showed that subpopulation sizes can be modulated in response to a challenge, for instance by LPS, MALP-2 and $\mathrm{Pam}_{3} \mathrm{CSK}_{4}$. In regard to LPS, this phenomenon was already confirmed in the context of microglial neurotransmitters and neurohormone responsiveness (Pannell et al., 2014b). There, LPS stimulation lead to an up- or downregulation of the responding microglia subpopulation sizes.

The effect on subpopulation size, however, experiences a ceiling effect at a certain point of concentration, depending on the respective cytokine. This ceiling effect might resemble a physiological limitation of the immune reaction in regard to LPS and other immunoreactive substances. In an intact central nervous immune system, this limitation could prevent an overproduction of cytokines, which would result in a disruption of homeostasis and finally neurodegeneration. As dysregulation of microglial cells is considered as part of the pathology in neurodegenerative diseases, this physiological limitation might be affected as well leading to exacerbated inflammatory responses (von Bernhardi et al., 2015).

Furthermore, I have shown that the increase in subpopulation size correlates with cytokine secretion. Hereby, the degree of influence strongly depends on the respective cytokine and might be limited to certain stimuli. Thus, the increases in cytokine release upon stronger challenges are caused by an increase in the amount of secreting microglia cells. Though studies in that field of research are sparse, a single study demonstrated a similar effect in mouse fibroblasts (Tay et al., 2010). There, single cell analysis of TNFa induced activation revealed that higher doses activate more cells than lower ones which supports the here demonstrated correlation. The authors were even able to develop a stochastic mathematical model which reproduces the demonstrated dynamics quite precisely. As microglia subpopulations behave similarly, it is likely that their dynamics can be fitted to a model as well, which would be a big step forward in understanding how this biological system operates.

Though I demonstrated a correlation between microglia subpopulation sizes and cytokine secretion, calculation of the cytokine secretion per cell revealed an increase as well, which seems to be cytokine- as well as stimuli-dependent. While the TNFa secretion per cell increased with increasing stimuli concentrations, the RANTES secretion rate altered upon LPS stimulation only. Interestingly, these findings are in line with macrophage research, showing that the transcription factor Gli-3 is expressed by a subpopulation of macrophages only (Ravasi et al., 2002). This 
subpopulation as well as the expression level per cell increased significantly upon LPS stimulation. Even though, these experiments were performed on mRNA and not on protein level, they endorse the previously described results.

In conclusion, the relationship between the subpopulation size and stimuli strength might be uniform while the cytokine secretion per cell is cytokine as well as stimuli specific. Thus, a general conclusion answering the initial question if an increase in the amount of cytokines is caused by an increase in secreting cells and/ or an increase in secretion per cell cannot be made. It is possible that there is just an increase in the number of microglia but it is also possible that there is an additional increase in the amount of secretion per cell. These results highlight the complex organization behind microglia subpopulations which seems to be stimulus as well as cytokine dependent. Of course, it cannot be excluded that there are also subpopulations that vary just in the secreted cytokine amount per cell, but to elucidate this additional research on other subpopulations is needed. Though, the results imply that microglia subpopulations are very plastic which would exclude the small possibility that microglia subsets are arising during a late step in development after tissue invasion. Of course, this origin would make it quite easy to identify subsets and subset-specific properties which could be targeted by a drug, but reality seems to be more complicated. 


\subsection{Microglia subpopulation sizes and behavior are density dependent}

Different brain regions are known to vary highly in their microglia numbers, which was demonstrated for mice as well as for humans (Lawson et al., 1990; Mittelbronn et al., 2001). Microglia numbers differ from $5 \%$ to $12 \%$ of all cells in the murine brain and from $0.5 \%$ to $16 \%$ in human. The distinct brain regions are known to display highly diverse characteristics, ranging from microglial gene expression profiles to receptor abundance (Crain and Watters, 2015; Grabert et al., 2016). Important factors that play a role in shaping these interregional differences, thereby affecting the microglia, are the microenvironment and the expression of immunoregulatory markers (de Haas et al., 2008; Ren et al., 1999; Schmid et al., 2009; Smith et al., 2013; Wu et al., 1997). Whether the density of microglia by itself contributes to this variation is yet unknown. Though there is evidence for a density dependent effect of microglia, as differences in their abundance affect LPS-induced neurotoxicity of neurons in vitro (Kim et al., 2000).

Thus, I hypothesized that microglia densities play a role in shaping microglia subpopulations as well. By investigating the TNFa- and RANTES- producing microglia subpopulation sizes in microglia of increasing densities, I demonstrated a negative correlation between subpopulation size and density upon LPS, MALP-2 or $\mathrm{Pam}_{3} \mathrm{CSK}_{4}$ stimulation.

I assumed that this dampening in microglia subpopulation size increase might be caused by a self-regulatory mechanism, preventing the microglia from TNFa overproduction. However, analysis revealed a positive correlation between density and secretion per cell as well. It can thus be concluded that denser microglia have a smaller proportion of TNFa producing cells, though, with a significantly higher relative TNFa production. As a result, denser microglia populations produce significantly more TNFa than populations of lower density when facing an equal threat. The potential inflammatory consequences of this phenomenon are intense as a shift in cell density by a factor of 3 might result in a fourfold higher cytokine concentration.

The relationship between microglia abundance and cytokine secretion, which likely applies for cytokines in general, has therefore a very strong impact on the force of the immune reaction, which appears to be more aggressive in higher densities. As microglia densities vary within 
brain regions, it could be hypothesized that the different susceptibilities to an immune challenge between the regions are caused by this density dependent intensity of the immune reaction. This hypothesis would fit to an observation made by Kim at al. (2000) as they described an increased LPS-induced neurotoxicity for increasing microglia:neuron ratios. An overproduction of cytokines is at certain concentrations toxic to neurons. The correlation between the microglia abundance and the increase in TNFa secretion might be a reasonable explanation. This hypothesis is supported by the fact, that microglia expand their number during chronic neurodegenerative disease progression, which is linked to an increased neuronal degeneration (Gómez-Nicola et al., 2013).

Interestingly, the described density dependence had no effect on the relative increase of the subpopulation size, which was similar between all tested densities. This observation would fit to a hypothesis made by Ravasi and Hume, who claimed that macrophage heterogeneity arises from transcriptional probability (Ravasi et al., 2002). Each gene would thus have a specific activation probability which rises upon challenges like LPS stimulation. Assuming that this hypothesis applies for microglia as well the uniform increase in subpopulation sizes regarding different cell densities might be caused by a static expression probability of the TNFa gene, regardless of the relative cell abundance.

It can thus be concluded that the microglia density has a huge effect on shaping microglia subpopulations in size and effect intensity, though not on the stimuli induced progression, which instead might be organized in a probabilistic manner. The blockage of microglia expansion could serve as a useful therapeutic approach, by limiting the microglia density, thereby delaying disease progression. Indeed, pharmacological treatment which inhibits microglial proliferation was just recently shown to prevent the progression of Alzheimer's-like pathology in mice (Olmos-Alonso et al., 2016). 


\subsection{Microglia subpopulations are highly complex organized}

Microglia perform a whole set of different functions. Recent research could demonstrate that they are organized in functional subpopulations to achieve this diversity (Marshall et al., 2014; Venkatesan et al., 2010). As demonstrated here and elsewhere, secretion of cytokines is performed by microglia subsets as well (Scheffel et al., 2012). However, how the secretion of multiple cytokines is organized is largely unknown. Only a single study gave evidence that TNFa and interleukin 1 beta are produced by largely non-overlapping microglia subpopulations after ischemic stroke in mice (Clausen et al., 2008). However, no further research addressing this topic has been conducted ever since. Thus and to gain further insight into that topic, I investigated the organization of TNFa and RANTES secreting subpopulations. Since both substances can be secreted by more than half of the microglia population, I hypothesized that specific subpopulations are likely to overlap. Analysis of unstimulated and LPS stimulated microglia subpopulations showed that, indeed, a microglia subset producing both TNFa and RANTES simultaneously exists. Cytokine-specific microglia were present as well, though behaved rather heterogeneous in response to a challenge, indicating an organization of those cells in even further subsets. While the proportion of TNFa positive and double positive microglia rose significantly upon stimulation, the RANTES positive population remained stable. Thus, it appears that there exists a more general microglia population, able to produce multiple cytokines and to adapt their size in response to challenges. In addition, there are more specialized subpopulations producing one cytokine only. Those subpopulations can, depending on the cytokine they produce, also adapt their size.

This complex organization of microglia subpopulations was also demonstrated by other studies in regard to receptor expression. Investigation of microglial CXCR4 and CXCR7 receptors revealed a subpopulation of $55 \%$ which co-expressed those receptors and subpopulations of 20 $\%$ and $6 \%$ expressing only CXCR4 or CXCR7 respectively (Lipfert et al., 2013). Consecutive application of galanin, somatostation and angiotensin II to neonatal mouse microglia resulted in microglia subsets of $31 \%$ responding to one stimulus, $8 \%$ to two stimuli and $2 \%$ that responded to all stimuli (Pannell et al., 2014b). They could also demonstrate a large number of microglia 
subpopulations expressing different subsets of neurotransmitter receptors leading to a huge diversity in their sensitivity.

In summary, cytokine secreting microglia subpopulations are composed of different subpopulations producing different cytokines or combinations, leading to heterogeneity within heterogeneity, finally resulting into an almost infinite organization complexity. My data support the possibility for transcriptional probability dependent heterogeneity. If LPS increases the possibility of certain genes to be expressed in each cell, a random landscape of microglia heterogeneity would emerge with each cell expressing different sets of genes. Giving our current knowledge on microglia heterogeneity, this complexity, however, makes a targeted drug development rather difficult, as specific cytokines are not exclusively produced by specific subsets but a combination of them. Investigating heterogeneity in microglia even further, identifying the whole variety within the "microgliome" is thus of utmost importance for future research. 


\subsection{Microglia are multitasking capable as they perform cy- tokine secretion and proliferation simultaneously}

So far, the capability of microglia to perform various different functions was termed multitasking (Casano and Peri, 2015). Multitasking, however, would indicate that microglia are able to perform several functions simultaneously, a feature which was not demonstrated for microglia so far, as most of the investigations were performed via bulk measurement. Investigation of microglia on single-cell level would be necessary to validate or disprove their multitasking abilities. Thus, I decided to investigate this issue by addressing proliferating microglia. As proliferation is one of the cells' most important and thus tightly controlled functions, it would be of great interest to know whether dividing microglia perform other tasks besides proliferation itself simultaneously. Therefore, I examined whether microglia are able to perform proliferation and TNFa secretion upon a challenge with LPS at once.

The effect of LPS on microglia proliferation is controversially issued in the literature. Microglia proliferation was shown to be either unaffected, increased, decreased or even both upon LPS stimulation, depending on the brain region (Chen et al., 2012; Fukushima et al., 2015; GebickeHaerter et al., 1989; George et al., 2015).

To gain further insight on this debate, I investigated the effect of LPS on microglia proliferation beforehand. The obtained results showed a reduction in the proliferation rate of microglia upon LPS stimulation, which confirms the findings of Gebicke-Haerter et al. (1989). This reduction, which was observed for immune challenges with $\mathrm{Pam}_{3} \mathrm{CSK}_{4}$ and fibronectin as well, might be initiated to prevent an excessive immune response caused by higher microglia densities. As microglia proliferation increases in chronic neurodegenerative diseases, this preventive mechanism is likely to become disrupted in those disorders.

Next, I investigated un- and LPS stimulated microglia for TNFa-secreting and proliferating subpopulations. Analysis revealed that microglia are able to perform both tasks simultaneously. Again, subpopulations positive for just one of the investigated functions were discovered as well. LPS stimulation lead, as expected, to an increase of the TNFa producing subpopulation. 
The subpopulations positive for both functions or proliferation only were not affected significantly. However, the previously observed reduction in microglia proliferation upon stimulation was confirmed in this approach as well.

Thus, it can be concluded that microglia are indeed capable of multitasking as some cells can proliferate and secrete cytokines at once. This capability might be a general feature, allowing the conduction of all possible functions simultaneously with at least a second one. This organization again supports the hypothesis that heterogeneity arises from transcriptional probability. 


\subsection{Microglia subpopulation size is influenced by environ- mental cues}

As microglia subpopulation sizes are dependent on the microglia density, the question arises how this effect is mediated. Since microglia have mostly non-overlapping territories a contactdependent organization is doubtful, leaving a substance-dependent organization as a possible alternative (Parkhurst and Gan, 2010). Since microglia functions are demonstrated to be influenced by the microenvironment, this explanation would be quite likely (Schmid et al., 2009; Wu et al., 1997).

Therefore, I hypothesized that microglia subpopulations are organized by one or more substances that are secreted by denser microglia in a heterogeneous manner. To proof this, I performed an insert experiment exposing microglia to supernatants from different microglia densities upon LPS stimulation. Unfortunately though, this method was not able to demonstrate any effect on the TNFa-producing microglia subpopulation size. Further research demonstrated that not all substances can pass through the insert membrane unhindered. Therefore, a more adequate experimental approach was needed for testing a supernatant induced effect.

To mimic a more accurate microenvironment, I thus performed a supernatant transfer experiment. Microglia, which were incubated with supernatants of increasing microglia densities, showed no density dependent differences in the TNFa- and RANTES-producing microglia subpopulation sizes. LPS stimulation which followed this supernatant transfer showed a similar outcome. These results indicate that the supernatant of unstimulated microglia has no effect, independent of the microglia density, suggesting a similar composition of the substances within the supernatants.

Microglia supplemented with supernatants of LPS stimulated microglia of increasing densities showed a reduced proportion of TNFa producing cells compared to microglia that were directly LPS stimulated. Thereby, the subpopulation sizes were independent from the density, presumably as the concentrations of signaling molecules already reached saturation. This effect was absent in RANTES-producing microglia, indicating a cytokine-specific regulation of subpopulations. 
This regulation is likely caused by an immune challenge and is not necessarily defined beforehand. However, further studies investigating a time course of regulatory signals are necessary to unravel a differential effect by microenvironment compositions and to clarify the underlying mechanisms. 


\subsection{Organization of microglia subpopulation sizes is partially self-regulated}

The demonstrated impact of the microenvironment on microglial subpopulations gave rise to new questions on the exact factors modulating this effect. Signaling of certain cytokines like IL6 leads to the suppression of its own production and/ or the production of other cytokines (Greenhalgh and Hilton, 2001). I hypothesized that cytokine signaling not only encompasses this negative feedback loop but also has an effect on the cytokine secreting subpopulation sizes.

To investigate this possibility, I examined the effect of TNFa on its own secretion and the secretion of other cytokines, namely IL6, IL12-p40, KC, RANTES, MIP1a and MCP-1 induced by LPS exposure.

The analysis revealed a striking difference between cytokine levels of LPS stimulated microglia and LPS stimulated microglia that were pre-stimulated with TNFa for more than 6 hours. The pre-incubation caused a marked reduction in IL6-, IL12-p40-, KC-, RANTES- and TNFa secretion, while the secretion of MIP1a was upregulated and the secretion of MCP-1 unaffected. As higher doses of TNFa were shown to induce increased productions of TNFa and IL6, suggesting a positive feedback loop, these results are quite contradicting (Kuno et al., 2005). One possible explanation might be the difference in initial TNFa concentrations and LPS stimulation, highlighting a dynamic self-regulation by TNFa rather than an unidirectional feedback loop.

Similar experiments using different cytokines were not able to induce a similar significant outcome. While RANTES had no effect at all, MCP-1 had just minor, non-stable effects on some cytokines. MIP1a elicited an increased release of RANTES, TNFa and its own, though, the differences were marginal compared to the previously described TNFa pre-incubation.

This outcome indicates that some cytokines like TNFa have a special status as they are able to effectively regulate the secretion of other cytokines. Correspondingly, microglia activation upon LPS is partially mediated by the microglia-derived TNFa and in liver cells, TNFa regulates chemokine induction after infection with mycobacterium tuberculosis (Kuno et al., 2005; Roach et al., 2002). No evidence is given by the literature for regulatory effects of RANTES, MCP-1 or 
MIP1 $\alpha$ on cytokine secretion, underlining the special status of TNFa.

Based on these results, TNFa seemed to be the most promising cytokine that could affect the cytokine secreting subpopulation size. Even though, the differences elicited by TNFa preincubation on the cytokine release could not be observed on mRNA level, a slight reduction in the TNFa producing subpopulation size was present at a pre-incubation time of 24 hours. The RANTES-producing subpopulation size was not affected by the pre-incubation. The quantitative difference between the secreted cytokines and subpopulation size regarding TNFa preincubation might be a consequence from a differential duration of LPS stimulation.

Nevertheless, a small effect of TNFa on the TNFa-producing microglia subpopulation size was demonstrated, indicating a self-regulating property of TNFa. This regulatory effect can likely be expanded to other cytokines as well as it is indicated by the ELISA data. To confirm this, further research is needed.

This self-regulation might explain the density caused differences in the TNFa-producing microglia subpopulation sizes. As microglia of a higher density produce much more TNFa than microglia of a lower density, the secreted TNFa could have a stronger effect on the microglia subpopulation size leading to a more pronounced reduction. Conclusively, the increased secretion would lead to a smaller subpopulation size in denser microglia than in less dense microglia which would fit to the observed density effect. 


\section{Summary and Conclusion}

In this study, I investigated the functional heterogeneity of microglia using cytokine secretion as a hallmark for an immune response. I was able to show that not all microglia but mere subpopulations secrete cytokines upon an immune challenge. Thereby, increasing severity of the challenge correlated with the subpopulation size and the amount of the secreted cytokines. The immune response was strongly cytokine and stimuli dependent, indicating an enormous plasticity of the subsets. This plasticity was not only demonstrated regarding the population size but also the cytokine secretion per cell, which was cytokine specifically regulated as well. Furthermore, I identified the microglial density as an additional factor shaping microglia subpopulations in size and efficiency. An increase in microglia density lead to a lower proportion of subpopulations and simultaneously to a higher cytokine production per cell, which finally results in a much stronger immune response. Interestingly, cell density has no effect on the stimuli induced progression of the subpopulation size increase, which instead might be organized in a probabilistic manner. The general effect of microglia density on the subpopulation size is likely caused by an immune challenge and not necessarily defined beforehand. This effect is most probably conveyed via secreted substances, as I could identify TNFa as one of them. TNFa has a regulatory effect on its own secretion and the secretion of other cytokines, which affects the cytokine producing subpopulation size as well. As TNFa in most cases leads to a reduction of the cytokine secretion, it might be considered as a mechanism to resolve the immune response.

Additionally, I was able to demonstrate the complex organization of subpopulations and their ability to perform multitasking. Thus, microglia subsets perform a variety of functions that might partially be shared by different subpopulations. This organization was identified for similar functions like secretion of different cytokines but also for different functions like cytokine secretion and proliferation.

Altogether, I could demonstrate that microglia can be categorized in functional subpopulations, which can be adapted as a response to a challenge. This adaptation might be highly specific as it depends on function, stimuli and microenvironment. Additionally, function specific subpopulations are composed of even more specific "subsubpopulations", which provides a small insight 
into the high complexity and the infinite possibilities of microglia subsets. Further investigation of microglia heterogeneity to identify the whole variety within the "microgliome" is thus of utmost importance for future research. 


\section{Bibliography}

Aarum, J., Sandberg, K., Haeberlein, S. L. B., Persson, M. A. A., Dec. 2003. Migration and differentiation of neural precursor cells can be directed by microglia. Proceedings of the National Academy of Sciences of the United States of America 100 (26), 15983-15988.

Adachi, O., Kawai, T., Takeda, K., Matsumoto, M., Tsutsui, H., Sakagami, M., Nakanishi, K., Akira, S., Jul. 1998. Targeted Disruption of the MyD88 Gene Results in Loss of IL-1- and IL-18-Mediated Function. Immunity 9 (1), 143-150.

Aguzzi, A., Barres, B. A., Bennett, M. L., Nov. 2013. Microglia: Scapegoat, Saboteur, or Something Else? Science 339 (6116), 156-161.

Ajami, B., Bennett, J. L., Krieger, C., Tetzlaff, W., Rossi, F. M. V., Dec. 2007. Local self-renewal can sustain CNS microglia maintenance and function throughout adult life. Nature Neuroscience 10 (12), 1538-1543.

Akira, S., Uematsu, S., Takeuchi, O., Feb. 2006. Pathogen Recognition and Innate Immunity. Cell 124 (4), 783-801.

Alexopoulou, L., Holt, A. C., Medzhitov, R., Flavell, R. A., Oct. 2001. Recognition of double-stranded RNA and activation of NF-kB by Toll-like receptor 3. Nature 413 (6857), 732-738.

Baalman, K., Marin, M. A., Ho, T. S.-Y., Godoy, M., Cherian, L., Robertson, C., Rasband, M. N., Feb. 2015. Axon initial segment-associated microglia. The Journal of Neuroscience: The Official Journal of the Society for Neuroscience 35 (5), 2283-2292.

Biber, K., Möller, T., Boddeke, E., Prinz, M., Feb. 2016. Central nervous system myeloid cells as drug targets: current status and translational challenges. Nature Reviews Drug Discovery 15 (2), 110-124.

Bisht, K., Sharma, K. P., Lecours, C., Gabriela Sánchez, M., El Hajj, H., Milior, G., Olmos-Alonso, A., GómezNicola, D., Luheshi, G., Vallières, L., Branchi, I., Maggi, L., Limatola, C., Butovsky, O., Tremblay, M.-v., Jan. 2016. Dark microglia: A new phenotype predominantly associated with pathological states. Glia, n/a-n/a.

Block, M. L., Hong, J.-S., Jun. 2005. Microglia and inflammation-mediated neurodegeneration: Multiple triggers with a common mechanism. Progress in Neurobiology 76 (2), 77-98.

Bruttger, J., Karram, K., Wörtge, S., Regen, T., Marini, F., Hoppmann, N., Klein, M., Blank, T., Yona, S., Wolf, Y., Mack, M., Pinteaux, E., Müller, W., Zipp, F., Binder, H., Bopp, T., Prinz, M., Jung, S., Waisman, A., Jul. 
2015. Genetic Cell Ablation Reveals Clusters of Local Self-Renewing Microglia in the Mammalian Central Nervous System. Immunity 43 (1), 92-106.

Butovsky, O., Koronyo-Hamaoui, M., Kunis, G., Ophir, E., Landa, G., Cohen, H., Schwartz, M., Aug. 2006. Glatiramer acetate fights against Alzheimer's disease by inducing dendritic-like microglia expressing insulinlike growth factor 1. Proceedings of the National Academy of Sciences of the United States of America 103 (31), 11784-11789.

Buttini, M., Appel, K., Sauter, A., Gebicke-Haerter, P. J., Boddeke, H. W. G. M., Mar. 1996. Expression of tumor necrosis factor alpha after focal cerebral ischaemia in the rat. Neuroscience 71 (1), 1-16.

Casano, A. M., Peri, F., Feb. 2015. Microglia: Multitasking Specialists of the Brain. Developmental Cell 32 (4), 469-477.

Chan, W. Y., Kohsaka, S., Rezaie, P., Feb. 2007. The origin and cell lineage of microglia-New concepts. Brain Research Reviews 53 (2), 344-354.

Chen, S.-K., Tvrdik, P., Peden, E., Cho, S., Wu, S., Spangrude, G., Capecchi, M. R., May 2010. Hematopoietic Origin of Pathological Grooming in Hoxb8 Mutant Mice. Cell 141 (5), 775-785.

Chen, Y., Won, S., Xu, Y., Swanson, R., 2014. Targeting Microglial Activation in Stroke Therapy: Pharmacological Tools and Gender Effects. Current medicinal chemistry 21 (19), 2146-2155.

Chen, Z., Jalabi, W., Shpargel, K. B., Farabaugh, K. T., Dutta, R., Yin, X., Kidd, G. J., Bergmann, C. C., Stohlman, S. A., Trapp, B. D., Aug. 2012. Lipopolysaccharide-Induced Microglial Activation and Neuroprotection against Experimental Brain Injury Is Independent of Hematogenous TLR4. The Journal of Neuroscience 32 (34), 11706-11715.

Clausen, B. H., Lambertsen, K. L., Babcock, A. A., Holm, T. H., Dagnaes-Hansen, F., Finsen, B., Oct. 2008. Interleukin-1beta and tumor necrosis factor-alpha are expressed by different subsets of microglia and macrophages after ischemic stroke in mice. Journal of Neuroinflammation 5, 46.

Crain, J. M., Watters, J. J., Dec. 2015. Microglial P2 Purinergic Receptor and Immunomodulatory Gene Transcripts Vary By Region, Sex, and Age in the Healthy Mouse CNS. Transcriptomics: open access 3 (2).

Cunningham, C. L., Martínez-Cerdeño, V., Noctor, S. C., Mar. 2013. Microglia regulate the number of neural precursor cells in the developing cerebral cortex. The Journal of neuroscience : the official journal of the Society for Neuroscience 33 (10), 4216-4233. 
Davalos, D., Grutzendler, J., Yang, G., Kim, J. V., Zuo, Y., Jung, S., Littman, D. R., Dustin, M. L., Gan, W.-B., Jun. 2005. ATP mediates rapid microglial response to local brain injury in vivo. Nature Neuroscience 8 (6), $752-758$.

de Haas, A. H., Boddeke, H. W. G. M., Biber, K., Jun. 2008. Region-specific expression of immunoregulatory proteins on microglia in the healthy CNS. Glia 56 (8), 888-894.

Elkabes, S., DiCicco-Bloom, E. M., Black, I. B., Apr. 1996. Brain microglia/macrophages express neurotrophins that selectively regulate microglial proliferation and function. The Journal of Neuroscience: The Official Journal of the Society for Neuroscience 16 (8), 2508-2521.

Elmore, M. P., Najafi, A., Koike, M., Dagher, N., Spangenberg, E., Rice, R., Kitazawa, M., Matusow, B., Nguyen, H., West, B., Green, K., Apr. 2014. Colony-Stimulating Factor 1 Receptor Signaling Is Necessary for Microglia Viability, Unmasking a Microglia Progenitor Cell in the Adult Brain. Neuron 82 (2), 380-397.

Fang, H., Chen, J., Lin, S., Wang, P., Wang, Y., Xiong, X., Yang, Q., Jun. 2014. CD36-Mediated Hematoma Absorption following Intracerebral Hemorrhage: Negative Regulation by TLR4 Signaling. The Journal of Immunology Author Choice 192 (12), 5984-5992.

Ferreira, R., Bernardino, L., Feb. 2015. Dual role of microglia in health and disease: pushing the balance toward repair. Frontiers in Cellular Neuroscience 9.

Ferrer, I., Bernet, E., Soriano, E., Del Rio, T., Fonseca, M., 1990. Naturally occurring cell death in the cerebral cortex of the rat and removal of dead cells by transitory phagocytes. Neuroscience 39 (2), 451-458.

Färber, K., Pannasch, U., Kettenmann, H., May 2005. Dopamine and noradrenaline control distinct functions in rodent microglial cells. Molecular and cellular neurosciences 29 (1), 128-138.

Fukushima, S., Furube, E., Itoh, M., Nakashima, T., Miyata, S., Aug. 2015. Robust increase of microglia proliferation in the fornix of hippocampal axonal pathway after a single LPS stimulation. Journal of Neuroimmunology $285,31-40$.

Gebicke-Haerter, P. J., Bauer, J., Schobert, A., Northoff, H., Jan. 1989. Lipopolysaccharide-free conditions in primary astrocyte cultures allow growth and isolation of microglial cells. The Journal of Neuroscience 9 (1), 183-194.

George, J., Gonçalves, F. Q., Cristóvão, G., Rodrigues, L., Meyer Fernandes, J. R., Gonçalves, T., Cunha, R. A., Gomes, C. A., Sep. 2015. Different danger signals differently impact on microglial proliferation through alterations of ATP release and extracellular metabolism. Glia 63 (9), 1636-1645. 
Gertig, U., Hanisch, U.-K., 2014. Microglial diversity by responses and responders. Frontiers in Cellular Neuroscience 8, 101.

Ginhoux, F., Greter, M., Leboeuf, M., Nandi, S., See, P., Gokhan, S., Mehler, M. F., Conway, S. J., Ng, L. G., Stanley, E. R., Samokhvalov, I. M., Merad, M., May 2010. Fate Mapping Analysis Reveals That Adult Microglia Derive from Primitive Macrophages. Science 330 (6005), 841-845.

Gómez-Nicola, D., Fransen, N. L., Suzzi, S., Perry, V. H., Jun. 2013. Regulation of Microglial Proliferation during Chronic Neurodegeneration. The Journal of Neuroscience 33 (6), 2481-2493.

Godbout, J. P., Chen, J., Abraham, J., Richwine, A. F., Berg, B. M., Kelley, K. W., Johnson, R. W., Aug. 2005. Exaggerated neuroinflammation and sickness behavior in aged mice following activation of the peripheral innate immune system. FASEB journal: official publication of the Federation of American Societies for Experimental Biology 19 (10), 1329-1331.

Gold, M., Khoury, J. E., Aug. 2015. $\beta$-amyloid, microglia, and the inflammasome in Alzheimer's disease. Seminars in Immunopathology 37 (6), 607-611.

Grabert, K., Michoel, T., Karavolos, M. H., Clohisey, S., Baillie, J. K., Stevens, M. P., Freeman, T. C., Summers, K. M., McColl, B. W., Jan. 2016. Microglial brain region-dependent diversity and selective regional sensitivities to aging. Nature Neuroscience advance online publication.

Graeber, M. B., Tetzlaff, W., Streit, W. J., Kreutzberg, G. W., Mar. 1988. Microglial cells but not astrocytes undergo mitosis following rat facial nerve axotomy. Neuroscience Letters 85 (3), 317-321.

Greenhalgh, C. J., Hilton, D. J., Jan. 2001. Negative regulation of cytokine signaling. Journal of Leukocyte Biology 70 (3), 348-356.

Hanisch, U.-K., Nov. 2002. Microglia as a source and target of cytokines. Glia 40 (2), 140-155.

Hanisch, U.-K., May 2013. Functional diversity of microglia - how heterogeneous are they to begin with? Frontiers in Cellular Neuroscience 7.

Hanisch, U.-K., Kettenmann, H., Nov. 2007. Microglia: active sensor and versatile effector cells in the normal and pathologic brain. Nature Neuroscience 10 (11), 1387-1394.

Hayashi, F., Smith, K. D., Ozinsky, A., Hawn, T. R., Yi, E. C., Goodlett, D. R., Eng, J. K., Akira, S., Underhill, D. M., Aderem, A., Apr. 2001. The innate immune response to bacterial flagellin is mediated by Toll-like receptor 5. Nature 410 (6832), 1099-1103. 
Haynes, S. E., Hollopeter, G., Yang, G., Kurpius, D., Dailey, M. E., Gan, W.-B., Julius, D., Dec. 2006. The P2y12 receptor regulates microglial activation by extracellular nucleotides. Nature Neuroscience 9 (12), 15121519.

Heil, F., Hemmi, H., Hochrein, H., Ampenberger, F., Kirschning, C., Akira, S., Lipford, G., Wagner, H., Bauer, S., Mar. 2004. Species-Specific Recognition of Single-Stranded RNA via Toll-like Receptor 7 and 8. Science 303 (5663), 1526-1529.

Hemmi, H., Kaisho, T., Takeuchi, O., Sato, S., Sanjo, H., Hoshino, K., Horiuchi, T., Tomizawa, H., Takeda, K., Akira, S., Feb. 2002. Small anti-viral compounds activate immune cells via the TLR7 MyD88-dependent signaling pathway. Nature Immunology 3 (2), 196-200.

Hemmi, H., Takeuchi, O., Kawai, T., Kaisho, T., Sato, S., Sanjo, H., Matsumoto, M., Hoshino, K., Wagner, H., Takeda, K., Akira, S., Dec. 2000. A Toll-like receptor recognizes bacterial DNA. Nature 408 (6813), 740-745.

Hickman, S. E., Allison, E. K., Khoury, J. E., Aug. 2008. Microglial dysfunction and defective $\beta$-amyloid clearance pathways in aging Alzheimer's disease mice. The Journal of neuroscience : the official journal of the Society for Neuroscience 28 (33), 8354-8360.

Himmerich, H., Schönherr, J., Fulda, S., Sheldrick, A. J., Bauer, K., Sack, U., Oct. 2011. Impact of antipsychotics on cytokine production in-vitro. Journal of Psychiatric Research 45 (10), 1358-1365.

Hoebe, K., Du, X., Georgel, P., Janssen, E., Tabeta, K., Kim, S. O., Goode, J., Lin, P., Mann, N., Mudd, S., Crozat, K., Sovath, S., Han, J., Beutler, B., Aug. 2003. Identification of Lps2 as a key transducer of MyD88independent TIR signalling. Nature 424 (6950), 743-748.

Hoeffel, G., Chen, J., Lavin, Y., Low, D., Almeida, F., See, P., Beaudin, A., Lum, J., Low, I., Forsberg, E. C., Poidinger, M., Zolezzi, F., Larbi, A., Ng, L., Chan, J. Y., Greter, M., Becher, B., Samokhvalov, I., Merad, M., Ginhoux, F., Apr. 2015. C-Myb+ Erythro-Myeloid Progenitor-Derived Fetal Monocytes Give Rise to Adult Tissue-Resident Macrophages. Immunity 42 (4), 665-678.

Honda, S., Sasaki, Y., Ohsawa, K., Imai, Y., Nakamura, Y., Inoue, K., Kohsaka, S., Mar. 2001. Extracellular ATP or ADP Induce Chemotaxis of Cultured Microglia through Gi/o-Coupled P2y Receptors. The Journal of Neuroscience 21 (6), 1975-1982.

Hoshiko, M., Arnoux, I., Avignone, E., Yamamoto, N., Audinat, E., Oct. 2012. Deficiency of the Microglial Receptor CX3cr1 Impairs Postnatal Functional Development of Thalamocortical Synapses in the Barrel Cortex. The Journal of Neuroscience 32 (43), 15106-15111. 
Hoshino, K., Takeuchi, O., Kawai, T., Sanjo, H., Ogawa, T., Takeda, Y., Takeda, K., Akira, S., Jan. 1999. Cutting Edge: Toll-Like Receptor 4 (TLR4)-Deficient Mice Are Hyporesponsive to Lipopolysaccharide: Evidence for TLR4 as the Lps Gene Product. The Journal of Immunology 162 (7), 3749-3752.

Janova, H., Böttcher, C., Holtman, I. R., Regen, T., van Rossum, D., Götz, A., Ernst, A.-S., Fritsche, C., Gertig, U., Saiepour, N., Gronke, K., Wrzos, C., Ribes, S., Rolfes, S., Weinstein, J., Ehrenreich, H., Pukrop, T., Kopatz, J., Stadelmann, C., Salinas-Riester, G., Weber, M. S., Prinz, M., Brück, W., Eggen, B. J., Boddeke, H. W., Priller, J., Hanisch, U.-K., Apr. 2016. CD14 is a key organizer of microglial responses to CNS infection and injury. Glia 64 (4), 635-649.

Jin, M. S., Kim, S. E., Heo, J. Y., Lee, M. E., Kim, H. M., Paik, S.-G., Lee, H., Lee, J.-O., Sep. 2007. Crystal Structure of the TLR1-TLR2 Heterodimer Induced by Binding of a Tri-Acylated Lipopeptide. Cell 130 (6), 1071-1082.

Kamigaki, M., Hide, I., Yanase, Y., Shiraki, H., Harada, K., Tanaka, Y., Seki, T., Shirafuji, T., Tanaka, S., Hide, M., Sakai, N., Feb. 2016. The Toll-like receptor 4-activated neuroprotective microglia subpopulation survives via granulocyte macrophage colony-stimulating factor and JAK2/STAT5 signaling. Neurochemistry International 93, 82-94.

Kato, T., Mizoguchi, Y., Monji, A., Horikawa, H., Suzuki, S. O., Seki, Y., Iwaki, T., Hashioka, S., Kanba, S., Jul. 2008. Inhibitory effects of aripiprazole on interferon- $\gamma$-induced microglial activation via intracellular Ca2+ regulation in vitro. Journal of Neurochemistry 106 (2), 815-825.

Kato, T., Monji, A., Hashioka, S., Kanba, S., May 2007. Risperidone significantly inhibits interferon- $\gamma$-induced microglial activation in vitro. Schizophrenia Research 92 (1-3), 108-115.

Kawahara, K., Yoshida, A., Koga, K., Yokoo, S., Kuniyasu, A., Gotoh, T., Sawada, M., Nakayama, H., Mar. 2009. Marked induction of inducible nitric oxide synthase and tumor necrosis factor- $\alpha$ in rat CD40+ microglia by comparison to CD40 - microglia. Journal of Neuroimmunology 208 (1-2), 70-79.

Kawai, T., Akira, S., May 2010. The role of pattern-recognition receptors in innate immunity: update on Toll-like receptors. Nature Immunology 11 (5), 373-384.

Kettenmann, H., Hanisch, U.-K., Noda, M., Verkhratsky, A., Apr. 2011. Physiology of Microglia. Physiological Reviews 91 (2), 461-553.

Kettenmann, H., Kirchhoff, F., Verkhratsky, A., Jan. 2013. Microglia: New Roles for the Synaptic Stripper. Neuron 77 (1), 10-18. 
Kielian, T., Mayes, P., Kielian, M., Sep. 2002. Characterization of microglial responses to Staphylococcus aureus: effects on cytokine, costimulatory molecule, and Toll-like receptor expression. Journal of Neuroimmunology 130 (1-2), 86-99.

Kierdorf, K., Erny, D., Goldmann, T., Sander, V., Schulz, C., Perdiguero, E. G., Wieghofer, P., Heinrich, A., Riemke, P., Hölscher, C., Müller, D. N., Luckow, B., Brocker, T., Debowski, K., Fritz, G., Opdenakker, G., Diefenbach, A., Biber, K., Heikenwalder, M., Geissmann, F., Rosenbauer, F., Prinz, M., Mar. 2013. Microglia emerge from erythromyeloid precursors via Pu.1- and Irf8-dependent pathways. Nature Neuroscience 16 (3), 273-280.

Kim, W.-G., Mohney, R. P., Wilson, B., Jeohn, G.-H., Liu, B., Hong, J.-S., Aug. 2000. Regional Difference in Susceptibility to Lipopolysaccharide-Induced Neurotoxicity in the Rat Brain: Role of Microglia. The Journal of Neuroscience 20 (16), 6309-6316.

Kluge, M., Schuld, A., Schacht, A., Himmerich, H., Dalal, M. A., Wehmeier, P. M., Hinze-Selch, D., Kraus, T., Dittmann, R. W., Pollmächer, T., Jan. 2009. Effects of clozapine and olanzapine on cytokine systems are closely linked to weight gain and drug-induced fever. Psychoneuroendocrinology 34 (1), 118-128.

Kobayashi, K., Imagama, S., Ohgomori, T., Hirano, K., Uchimura, K., Sakamoto, K., Hirakawa, A., Takeuchi, H., Suzumura, A., Ishiguro, N., Kadomatsu, K., Mar. 2013. Minocycline selectively inhibits M1 polarization of microglia. Cell Death \& Disease 4 (3), e525.

Kocur, M., Schneider, R., Pulm, A.-K., Bauer, J., Kropp, S., Gliem, M., Ingwersen, J., Goebels, N., Alferink, J., Prozorovski, T., Aktas, O., Scheu, S., Apr. 2015. IFN $\beta$ secreted by microglia mediates clearance of myelin debris in CNS autoimmunity. Acta Neuropathologica Communications 3.

Koizumi, S., Shigemoto-Mogami, Y., Nasu-Tada, K., Shinozaki, Y., Ohsawa, K., Tsuda, M., Joshi, B. V., Jacobson, K. A., Kohsaka, S., Inoue, K., Apr. 2007. UDP acting at P2y6 receptors is a mediator of microglial phagocytosis. Nature 446 (7139), 1091-1095.

Kowalski, J., Labuzek, K., Herman, Z. S., Jul. 2003. Flupentixol and trifluperidol reduce secretion of tumor necrosis factor- $a$ and nitric oxide by rat microglial cells. Neurochemistry International 43 (2), 173-178.

Kuhn, S. A., van Landeghem, F. K. H., Zacharias, R., Färber, K., Rappert, A., Pavlovic, S., Hoffmann, A., Nolte, C., Kettenmann, H., Feb. 2004. Microglia express GABAB receptors to modulate interleukin release. Molecular and Cellular Neuroscience 25 (2), 312-322.

Kuno, R., Wang, J., Kawanokuchi, J., Takeuchi, H., Mizuno, T., Suzumura, A., May 2005. Autocrine activation of microglia by tumor necrosis factor- $a$. Journal of Neuroimmunology 162 (1-2), 89-96. 
Lawson, L. J., Perry, V. H., Dri, P., Gordon, S., 1990. Heterogeneity in the distribution and morphology of microglia in the normal adult mouse brain. Neuroscience 39 (1), 151-170.

Lipfert, J., Ödemis, V., Wagner, D.-C., Boltze, J., Engele, J., Oct. 2013. CXCR4 and CXCR7 form a functional receptor unit for SDF-1/CXCL12 in primary rodent microglia. Neuropathology and Applied Neurobiology 39 (6), 667-680.

Liu, G. J., Nagarajah, R., Banati, R. B., Bennett, M. R., Mar. 2009. Glutamate induces directed chemotaxis of microglia. European Journal of Neuroscience 29 (6), 1108-1118.

London, A., Benhar, I., Mattapallil, M. J., Mack, M., Caspi, R. R., Schwartz, M., Jan. 2013. Functional Macrophage Heterogeneity in a Mouse Model of Autoimmune Central Nervous System Pathology. The Journal of Immunology 190 (7), 3570-3578.

Lund, S., Christensen, K. V., Hedtjärn, M., Mortensen, A. L., Hagberg, H., Falsig, J., Hasseldam, H., Schrattenholz, A., Pörzgen, P., Leist, M., Nov. 2006. The dynamics of the LPS triggered inflammatory response of murine microglia under different culture and in vivo conditions. Journal of Neuroimmunology 180 (1-2), 71-87.

Maezawa, I., Jin, L.-W., Apr. 2010. Rett Syndrome Microglia Damage Dendrites and Synapses by the Elevated Release of Glutamate. The Journal of Neuroscience 30 (15), 5346-5356.

Marshall, G. P., Deleyrolle, L. P., Reynolds, B. A., Steindler, D. A., Laywell, E. D., Jul. 2014. Microglia from neurogenic and non-neurogenic regions display differential proliferative potential and neuroblast support. Frontiers in Cellular Neuroscience 8.

Masuda, T., Prinz, M., Feb. 2016. Microglia: A Unique Versatile Cell in the Central Nervous System. ACS Chemical Neuroscience.

Meeuwsen, S., Bsibsi, M., Persoon-Deen, C., Ravid, R., van Noort, J. M., 2005. Cultured human adult microglia from different donors display stable cytokine, chemokine and growth factor gene profiles but respond differently to a pro-inflammatory stimulus. Neuroimmunomodulation 12 (4), 235-245.

Mildner, A., Schmidt, H., Nitsche, M., Merkler, D., Hanisch, U.-K., Mack, M., Heikenwalder, M., Brück, W., Priller, J., Prinz, M., Dec. 2007. Microglia in the adult brain arise from Ly-6chiccr2+ monocytes only under defined host conditions. Nature Neuroscience 10 (12), 1544-1553.

Mittelbronn, M., Dietz, K., Schluesener, H. J., Meyermann, R., Mar. 2001. Local distribution of microglia in the normal adult human central nervous system differs by up to one order of magnitude. Acta Neuropathologica 101 (3), 249-255. 
Nimmerjahn, A., Kirchhoff, F., Helmchen, F., May 2005. Resting Microglial Cells Are Highly Dynamic Surveillants of Brain Parenchyma in Vivo. Science 308 (5726), 1314-1318.

Noda, M., Nakanishi, H., Nabekura, J., Akaike, N., Jan. 2000. AMPA-Kainate Subtypes of Glutamate Receptor in Rat Cerebral Microglia. The Journal of Neuroscience 20 (1), 251-258.

Olah, M., Amor, S., Brouwer, N., Vinet, J., Eggen, B., Biber, K., Boddeke, H. W. G. M., Feb. 2012. Identification of a microglia phenotype supportive of remyelination. Glia 60 (2), 306-321.

Olmos-Alonso, A., Schetters, S. T. T., Sri, S., Askew, K., Mancuso, R., Vargas-Caballero, M., Holscher, C., Perry, V. H., Gomez-Nicola, D., Jan. 2016. Pharmacological targeting of CSF1r inhibits microglial proliferation and prevents the progression of Alzheimer's-like pathology. Brain, awv379.

Ozinsky, A., Underhill, D. M., Fontenot, J. D., Hajjar, A. M., Smith, K. D., Wilson, C. B., Schroeder, L., Aderem, A., Dec. 2000. The repertoire for pattern recognition of pathogens by the innate immune system is defined by cooperation between Toll-like receptors. Proceedings of the National Academy of Sciences of the United States of America 97 (25), 13766-13771.

Pannell, M., Meier, M. A., Szulzewsky, F., Matyash, V., Endres, M., Kronenberg, G., Prinz, V., Waiczies, S., Wolf, S. A., Kettenmann, H., Dec. 2014a. The subpopulation of microglia expressing functional muscarinic acetylcholine receptors expands in stroke and Alzheimer's disease. Brain Structure and Function, 1-16.

Pannell, M., Szulzewsky, F., Matyash, V., Wolf, S. A., Kettenmann, H., 2014b. The subpopulation of microglia sensitive to neurotransmitters/neurohormones is modulated by stimulation with LPS, interferon- $\gamma$, and IL-4. Glia, n/a-n/a.

Paolicelli, R. C., Bolasco, G., Pagani, F., Maggi, L., Scianni, M., Panzanelli, P., Giustetto, M., Ferreira, T. A., Guiducci, E., Dumas, L., Ragozzino, D., Gross, C. T., Sep. 2011. Synaptic Pruning by Microglia Is Necessary for Normal Brain Development. Science 333 (6048), 1456-1458.

Parkhurst, C. N., Gan, W.-B., Oct. 2010. Microglia dynamics and function in the CNS. Current opinion in neurobiology 20 (5), 595-600.

Parkhurst, C. N., Yang, G., Ninan, I., Savas, J. N., Yates, J. R., Lafaille, J. J., Hempstead, B. L., Littman, D. R., Gan, W.-B., Dec. 2013. Microglia promote learning-dependent synapse formation through BDNF. Cell 155 (7), 1596-1609.

Pepe, G., Calderazzi, G., De Maglie, M., Villa, A. M., Vegeto, E., Dec. 2014. Heterogeneous induction of microglia M2a phenotype by central administration of interleukin-4. Journal of Neuroinflammation 11. 
Peri, F., Nüsslein-Volhard, C., May 2008. Live Imaging of Neuronal Degradation by Microglia Reveals a Role for v0-ATPase a1 in Phagosomal Fusion In Vivo. Cell 133 (5), 916-927.

Poltorak, A., He, X., Smirnova, I., Liu, M.-Y., Huffel, C. V., Du, X., Birdwell, D., Alejos, E., Silva, M., Galanos, C., Freudenberg, M., Ricciardi-Castagnoli, P., Layton, B., Beutler, B., Dec. 1998. Defective LPS Signaling in C3h/HeJ and C57bl/10sccr Mice: Mutations in Tlr4 Gene. Science 282 (5396), 2085-2088.

Prinz, M., Mildner, A., Feb. 2011. Microglia in the CNS: Immigrants from another world. Glia 59 (2), $177-187$.

Prokop, S., Miller, K. R., Heppner, F. L., Oct. 2013. Microglia actions in Alzheimer's disease. Acta Neuropathologica $126(4), 461-477$.

Ravasi, T., Wells, C., Forest, A., Underhill, D. M., Wainwright, B. J., Aderem, A., Grimmond, S., Hume, D. A., Jan. 2002. Generation of Diversity in the Innate Immune System: Macrophage Heterogeneity Arises from Gene-Autonomous Transcriptional Probability of Individual Inducible Genes. The Journal of Immunology 168 (1), 44-50.

Regen, T., van Rossum, D., Scheffel, J., Kastriti, M.-E., Revelo, N. H., Prinz, M., Brück, W., Hanisch, U.-K., Jul. 2011. CD14 and TRIF govern distinct responsiveness and responses in mouse microglial TLR4 challenges by structural variants of LPS. Brain, Behavior, and Immunity 25 (5), 957-970.

Ren, L.-q., Lubrich, B., Biber, K., Gebicke-Haerter, P. J., Mar. 1999. Differential expression of inflammatory mediators in rat microglia cultured from different brain regions. Molecular Brain Research 65 (2), 198205.

Ritzel, R. M., Patel, A. R., Pan, S., Crapser, J., Hammond, M., Jellison, E., McCullough, L. D., Jun. 2015. Ageand location-related changes in microglial function. Neurobiology of Aging 36 (6), 2153-2163.

Roach, D. R., Bean, A. G. D., Demangel, C., France, M. P., Briscoe, H., Britton, W. J., Jan. 2002. TNF Regulates Chemokine Induction Essential for Cell Recruitment, Granuloma Formation, and Clearance of Mycobacterial Infection. The Journal of Immunology 168 (9), 4620-4627.

Saijo, K., Glass, C. K., Nov. 2011. Microglial cell origin and phenotypes in health and disease. Nature Reviews Immunology 11 (11), 775-787.

Schafer, D., Lehrman, E., Kautzman, A., Koyama, R., Mardinly, A., Yamasaki, R., Ransohoff, R., Greenberg, M., Barres, B., Stevens, B., May 2012. Microglia Sculpt Postnatal Neural Circuits in an Activity and Complement-Dependent Manner. Neuron 74 (4), 691-705.

Schafer, D. P., Stevens, B., Jan. 2015. Microglia Function in Central Nervous System Development and Plasticity. Cold Spring Harbor Perspectives in Biology 7 (10), a020545. 
Scheffel, J., Regen, T., Van Rossum, D., Seifert, S., Ribes, S., Nau, R., Parsa, R., Harris, R. A., Boddeke, H. W. G. M., Chuang, H.-N., Pukrop, T., Wessels, J. T., Jürgens, T., Merkler, D., Brück, W., Schnaars, M., Simons, M., Kettenmann, H., Hanisch, U.-K., 2012. Toll-like receptor activation reveals developmental reorganization and unmasks responder subsets of microglia. Glia 60 (12), 1930-1943.

Schmid, C. D., Melchior, B., Masek, K., Puntambekar, S. S., Danielson, P. E., Lo, D. D., Gregor Sutcliffe, J., Carson, M. J., May 2009. Differential gene expression in LPS/IFN $\gamma$ activated microglia and macrophages: in vitro versus in vivo. Journal of Neurochemistry 109 (Suppl. 1), 117-125.

Schmid, C. D., Sautkulis, L. N., Danielson, P. E., Cooper, J., Hasel, K. W., Hilbush, B. S., Sutcliffe, J. G., Carson, M. J., Dec. 2002. Heterogeneous expression of the triggering receptor expressed on myeloid cells-2 on adult murine microglia. Journal of Neurochemistry 83 (6), 1309-1320.

Schromm, A. B., Lien, E., Henneke, P., Chow, J. C., Yoshimura, A., Heine, H., Latz, E., Monks, B. G., Schwartz, D. A., Miyake, K., Golenbock, D. T., Jul. 2001. Molecular Genetic Analysis of an Endotoxin Nonresponder Mutant Cell Line. The Journal of Experimental Medicine 194 (1), 79-88.

Schwandner, R., Dziarski, R., Wesche, H., Rothe, M., Kirschning, C. J., Jun. 1999. Peptidoglycan- and Lipoteichoic Acid-induced Cell Activation Is Mediated by Toll-like Receptor 2. Journal of Biological Chemistry 274 (25), 17406-17409.

Shigemoto-Mogami, Y., Hoshikawa, K., Goldman, J. E., Sekino, Y., Sato, K., Feb. 2014. Microglia Enhance Neurogenesis and Oligodendrogenesis in the Early Postnatal Subventricular Zone. The Journal of Neuroscience 34 (6), 2231-2243.

Shimazu, R., Akashi, S., Ogata, H., Nagai, Y., Fukudome, K., Miyake, K., Kimoto, M., Jun. 1999. MD-2, a Molecule that Confers Lipopolysaccharide Responsiveness on Toll-like Receptor 4. The Journal of Experimental Medicine 189 (11), 1777-1782.

Shinjo, R., Imagama, S., Ito, Z., Ando, K., Nishida, Y., Ishiguro, N., Kadomatsu, K., Sep. 2014. Keratan sulfate expression is associated with activation of a subpopulation of microglia/macrophages in Wallerian degeneration. Neuroscience Letters 579, 80-85.

Sierra, A., Encinas, J. M., Deudero, J. J. P., Chancey, J. H., Enikolopov, G., Overstreet-Wadiche, L. S., Tsirka, S. E., Maletic-Savatic, M., Oct. 2010. Microglia Shape Adult Hippocampal Neurogenesis through ApoptosisCoupled Phagocytosis. Cell Stem Cell 7 (4), 483-495.

Sierra, A., Gottfried-Blackmore, A. C., McEwen, B. S., Bulloch, K., Mar. 2007. Microglia derived from aging mice exhibit an altered inflammatory profile. Glia 55 (4), 412-424. 
Smith, S. M. C., Friedle, S. A., Watters, J. J., Dec. 2013. Chronic Intermittent Hypoxia Exerts CNS RegionSpecific Effects on Rat Microglial Inflammatory and TLR4 Gene Expression. PLoS ONE 8 (12).

Squarzoni, P., Oller, G., Hoeffel, G., Pont-Lezica, L., Rostaing, P., Low, D., Bessis, A., Ginhoux, F., Garel, S., Sep. 2014. Microglia Modulate Wiring of the Embryonic Forebrain. Cell Reports 8 (5), 1271-1279.

Streit, W. J., Nov. 2002. Microglia as neuroprotective, immunocompetent cells of the CNS. Glia 40 (2), $133-$ 139.

Streit, W. J., Sep. 2006. Microglial senescence: does the brain’s immune system have an expiration date? Trends in Neurosciences 29 (9), 506-510.

Streit, W. J., Graeber, M. B., Jan. 1993. Heterogeneity of microglial and perivascular cell populations: Insights gained from the facial nucleus paradigm. Glia 7 (1), 68-74.

Streit, W. J., Graeber, M. B., Kreutzberg, G. W., Feb. 1989. Peripheral nerve lesion produces increased levels of major histocompatibility complex antigens in the central nervous system. Journal of Neuroimmunology 21 (2-3), 117-123.

Sugino, H., Futamura, T., Mitsumoto, Y., Maeda, K., Marunaka, Y., Mar. 2009. Atypical antipsychotics suppress production of proinflammatory cytokines and up-regulate interleukin-10 in lipopolysaccharidetreated mice. Progress in Neuro-Psychopharmacology and Biological Psychiatry 33 (2), 303-307.

Suzuki K, Sugihara G, Ouchi Y, et al, Jan. 2013. MIcroglial activation in young adults with autism spectrum disorder. JAMA Psychiatry 70 (1), 49-58.

Takeda, K., Akira, S., Feb. 2004. TLR signaling pathways. Seminars in Immunology 16 (1), 3-9.

Takeuchi, O., Hoshino, K., Kawai, T., Sanjo, H., Takada, H., Ogawa, T., Takeda, K., Akira, S., Oct. 1999. Differential Roles of TLR2 and TLR4 in Recognition of Gram-Negative and Gram-Positive Bacterial Cell Wall Components. Immunity 11 (4), 443-451.

Tay, S., Hughey, J. J., Lee, T. K., Lipniacki, T., Quake, S. R., Covert, M. W., Jul. 2010. Single-cell NF-kB dynamics reveal digital activation and analogue information processing. Nature 466 (7303), 267-271.

Tremblay, M.-v., Lowery, R. L., Majewska, A. K., Nov. 2010. Microglial Interactions with Synapses Are Modulated by Visual Experience. PLoS Biology 8 (11).

Trias, E., Díaz-Amarilla, P., Olivera-Bravo, S., Isasi, E., Drechsel, D. A., Lopez, N., Bradford, C. S., Ireton, K. E., Beckman, J. S., Barbeito, L., Dec. 2013. Phenotypic transition of microglia into astrocyte-like cells associated with disease onset in a model of inherited ALS. Frontiers in Cellular Neuroscience 7. 
Ueno, M., Fujita, Y., Tanaka, T., Nakamura, Y., Kikuta, J., Ishii, M., Yamashita, T., May 2013. Layer V cortical neurons require microglial support for survival during postnatal development. Nature Neuroscience 16 (5), 543-551.

Venkatesan, C., Chrzaszcz, M., Choi, N., Wainwright, M. S., May 2010. Chronic upregulation of activated microglia immunoreactive for galectin-3/Mac-2 and nerve growth factor following diffuse axonal injury. Journal of Neuroinflammation 7 (1), 32.

von Bernhardi, R., Eugenín-von Bernhardi, L., Eugenín, J., Jul. 2015. Microglial cell dysregulation in brain aging and neurodegeneration. Frontiers in Aging Neuroscience 7.

Wake, H., Moorhouse, A. J., Jinno, S., Kohsaka, S., Nabekura, J., Jan. 2009. Resting Microglia Directly Monitor the Functional State of Synapses In Vivo and Determine the Fate of Ischemic Terminals. The Journal of Neuroscience 29 (13), 3974-3980.

Wes, P. D., Sayed, F. A., Bard, F., Gan, L., Oct. 2016. Targeting microglia for the treatment of Alzheimer's Disease. Glia 64 (10), 1710-1732.

Wlodarczyk, A., Cédile, O., Jensen, K. N., Jasson, A., Mony, J. T., Khorooshi, R., Owens, T., Sep. 2015. Pathologic and Protective Roles for Microglial Subsets and Bone Marrow- and Blood-Derived Myeloid Cells in Central Nervous System Inflammation. Frontiers in Immunology 6.

Wu, C. H., Chien, H. F., Chang, C. Y., Ling, E. A., May 1997. Heterogeneity of antigen expression and lectin labeling on microglial cells in the olfactory bulb of adult rats. Neuroscience Research 28 (1), 67-75.

Xavier, A. L. R., Kress, B. T., Goldman, S. A., Menezes, J. R. L. d., Nedergaard, M., Aug. 2015. A Distinct Population of Microglia Supports Adult Neurogenesis in the Subventricular Zone. The Journal of Neuroscience 35 (34), 11848-11861.

Yao, Y., Echeverry, S., Shi, X. Q., Yang, M., Yang, Q. Z., Wang, G. Y. F., Chambon, J., Wu, Y. C., Fu, K. Y., De Koninck, Y., Zhang, J., Mar. 2016. Dynamics of spinal microglia repopulation following an acute depletion. Scientific Reports 6.

Zhan, Y., Paolicelli, R. C., Sforazzini, F., Weinhard, L., Bolasco, G., Pagani, F., Vyssotski, A. L., Bifone, A., Gozzi, A., Ragozzino, D., Gross, C. T., Mar. 2014. Deficient neuron-microglia signaling results in impaired functional brain connectivity and social behavior. Nature Neuroscience 17 (3), 400-406.

Ziv, Y., Ron, N., Butovsky, O., Landa, G., Sudai, E., Greenberg, N., Cohen, H., Kipnis, J., Schwartz, M., Feb. 2006. Immune cells contribute to the maintenance of neurogenesis and spatial learning abilities in adulthood. Nature Neuroscience 9 (2), 268-275. 


\section{Curriculum vitae}

\section{Ulla Gertig, née Haladyniak}

date of birth December 02, 1988 in Burg

\section{Education}

10/2012 - 12/2016 Georg-August-University Göttingen

PhD in the graduate program "Molecular Medicine"

Constitutive heterogeneity and response diversity of microglia in pathological conditions

10/2010 - 09/2012 Georg-August-University Göttingen

M.Sc.: "Developmental, Neural, and Behavioral Biology”

Study focus: neurobiology

Effects of pharmacological inhibition of histone deacetylases in Drosophila olfactory memory (An age-dependent analysis)

10/2007 - 08/2010 Georg-August-University Göttingen

B.Sc.: Biology

The role of Igf2 signaling during the extinction of learned fear

08/2001 - 06/2007 Burger Roland-Gymnasium

Allgemeine Hochschulreife

\section{Scientific experience}

10/2012 - 12/2016 University Medical Center Göttingen

Institute of neuropathology

PhD student

03/2012 - 04/2012

Georg-August-University Göttingen

$02 / 2011-03 / 2011$

Institute for zoology and anthropology

04/2011 - 05/2011

Student assistant

$02 / 2010-03 / 2010$ 